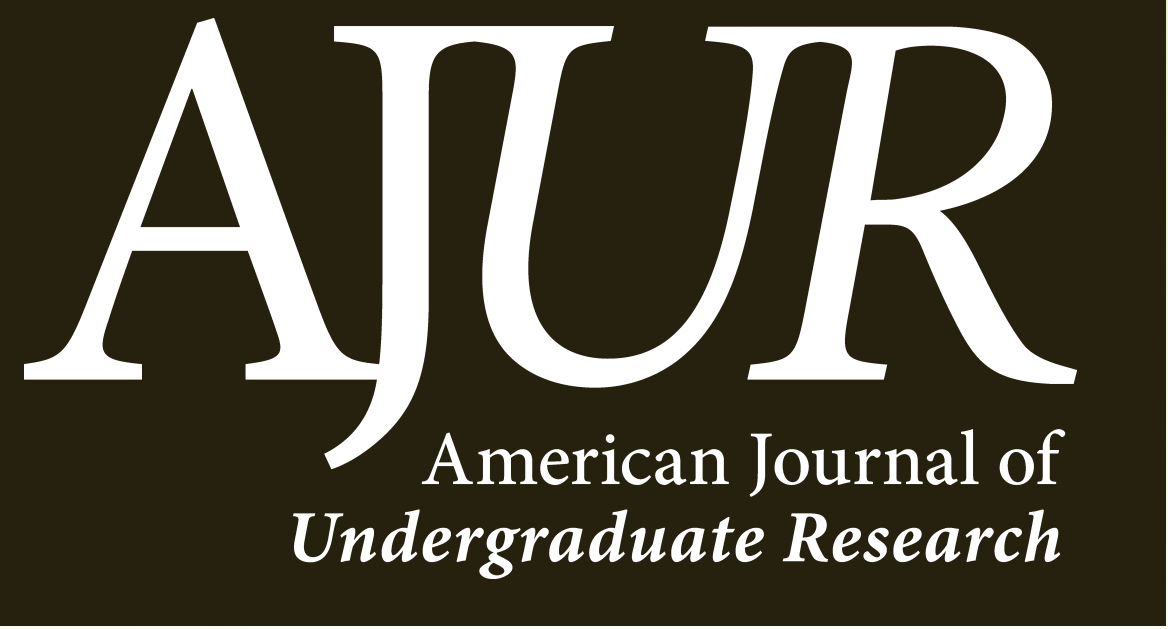

Volume 16 | Issue 1 | June 2019

www.ajuronline.org 


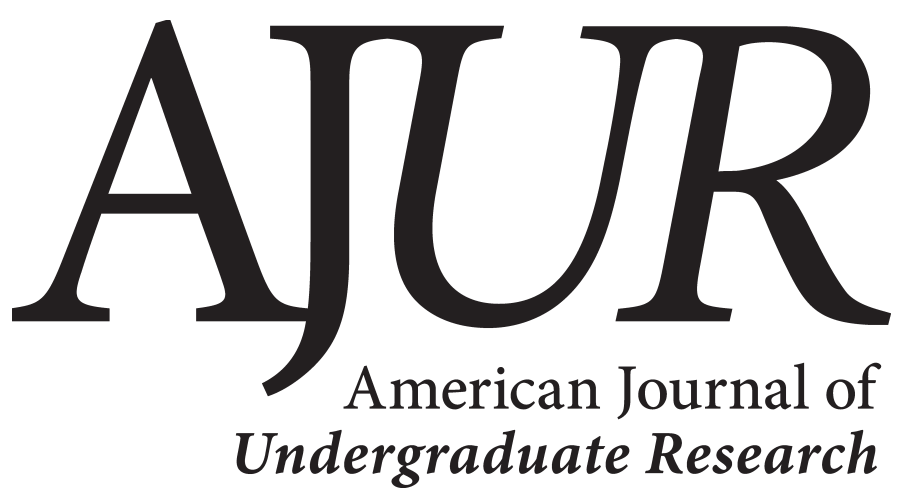

Volume 16 | Issue 1 | June 2019

www.ajuronline.org

AJUR History and Editorial Board

Special Thanks to AJUR's Sponsors

5 Should high-top or low-top cleats be used when playing baseball?

Margo P. Woblfeil, Cole R. Neary, Mitchell M. Klocke, Johnny G Mills, Rich C. Calvert, \&

David S. Senchina

Transcription of nanos-1 in Zebrafish Embryos is not Affected by Bisphenol A: Evaluated Using Quantitative Real-Time PCR

Bridget Babich, George Roba, Siti Sarah Safura*, Kevin Callahan, \& Edward Freeman

Investigation of Atmospheric Aerosol Size Distributions from

Ground-Based Solar Spectrometer Measurements Synthesized with

Satellite Data

Dane T. Kubr \& Adam T. Whitten

33 Diol Mediated Tautomerization of Glycine: a DFT Study

Francis Sub, Vanessa Rivera, \& Ruben D. Parra

41 Psychological Constructs as Predictors of Strength Gains in a Strength Training Course

Kathrine Hadley \& Joanna Morrissey

$49 \quad$ Modeling Networks of Evolving Populations

Sean Elliott

65 Assessing the Use of Cooking Demonstrations on Healthy Eating Barriers in City Bus Riders

Julianne Evans \& Diana Cuy Castellanos 
American Journal of Undergraduate Research (AJUR) is a national, independent, peer-reviewed, open-source, quarterly, multidisciplinary student research journal. Each manuscript of AJUR receives a DOI number. AJUR is archived by the US Library of Congress. AJUR was established in 2002, incorporated as an NFP in 2018. AJUR is indexed internationally by EBSCO and Crossref with ISSNs of 1536-4585 (print) and 2375-8732 (web).

EDITORIAL TEAM Volume 16 / Issue 1 / June 2019

Dr. Kestutis G. Bendinskas, Executive Editor, editor@ajuronline.org

Dr. Anthony Contento, Copy Editor, Treasurer

Peter Newell, Editor, Secretary

Daniel Laird, Web Master

EDITORIAL BOARD by subject area

\section{ACCOUNTING \\ Dr. Dean Crawford, dean.crawford@oswego.edu ARCHEOLOGY \\ Dr. Richard Redding, rredding@umich.edun \\ ART HISTORY \\ Dr. Lisa Seppi, lisa.seppi@oswego.edu ASTROPHYSICS \\ Dr. Shashi Kanbur, shasbi.kanbur@oswego.edu}

BEHAVIORAL NEUROSCIENCE

Dr. Aileen M. Bailey, ambailey@smom.edu BIOCHEMISTRY

Dr. Pamela K. Kerrigan, pamela.kerrigan@mountsaintvincent.edu

Dr. Nin Dingra, ndingra@alaska.edu BIOENGINEERING

Dr. Jorge I. Rodriguez, jorger@clemson.edu

Dr. Jessica Amber Jennings, jjnnings@memphis.edu

BIOINFORMATICS

Dr. Kevin Daimi,

daimikj@udmercy.edu

Dr. John R. Jungck, jungck@udel.edu

Dr. Isabelle Bichindaritz, ibicbind@oswego.edu

BIOLOGY, PHYSIOLOGY

Dr. David Dunn,

david.dunn@oswego.edu

BIOLOGY, DEVELOPMENTAL

Dr. Poongodi Geetha-Loganathan,

p.geethaloganathan@oswego.edu

BIOLOGY, MICROBIOLOGY

Dr. Peter Newell,

peternewell@oswego.edu BOTANY

Dr. William R. Bromer, wbromer@stfrancis.edu

Dr. Julien Bachelier,

julien.bacbelier@fu-berlin.de

\section{CHEMISTRY}

Dr. Alfredo Castro,

castroa@felician.edu

Dr. Charles Kriley, cekriley@gcc.edu

Dr. Douglas Mulford, douglas.mulford@emory.edu

Dr. Vadoud Niri,

vadoud.niri@oswego.edu

COMMUNICATION DISORDERS AND SCIENCES

Dr. Kim Tillery,

Kim.Tillery@fredonia.edu

COMPUTER SCIENCES

Dr. Dele Oluwade,

deleolunade@yaboo.com

Dr. Kevin Daimi,

daimikj@udmercy.edu

Dr. Levent Ertaul,

levent.ertaul@.csueastbay.edu

Dr. Mais W Nijim,

Mais.Nijim@tamuk.edu

COMPUTATIONAL CHEMISTRY

Dr. Alexander Soudackov

alexander.soudackov@yale.edu ECOLOGY

Dr. William R. Bromer, wbromer@stfrancis.edu ECONOMICS

Dr. Elizabeth Schmitt, elizabeth.schmitt@oswego.edu EDUCATION

Dr. Marcia Burrell,

marcia.burrell@oswego.edu

EDUCATION, PHYSICS

Dr. Andrew D. Gavrin, agavrin@iupui.edu

ENGINEERING, ELECTRICAI

Dr. Michael Omidiora,

michael.omidiora@nyu.edu

ENGINEERING, ENVIRONMENTAL

Dr. Eileen M. Cashman,

eileen.casbman@bumboldt.edu

\author{
ENGINEERING, SOFTWARE \\ Dr. Kevin Daimi, \\ daimikj@udmercy.edu \\ ENVIRONMENTAL SCIENCES \\ Dr. Eileen M. Cashman, \\ eilleen.casbman@bumboldt.edu \\ FILM AND MEDIA STUDIES \\ Dr. Lauren Steimer, \\ lsteimer@mailbox.sc.edu \\ GEOLOGY \\ Dr. Rachel Lee, \\ rachel.lee@oswego.edu \\ HISTORY \\ Dr. Richard Weyhing, \\ richard.weybing@oswego.edu \\ Dr. Murat Yasar, \\ murat.yasar@oswego.edu \\ HONORARY EDITORIAL \\ BOARD MEMBER \\ Dr. Lorrie Clemo, \\ lorrie.a.clemo@gmail.com \\ JURISPRUDENCE \\ Bill Wickard, Esq, \\ William.Wickard@KLGates.com \\ KINESIOLOGY \\ Dr. David Senchina, \\ david.sencbina@drake.edu \\ LITERARY STUDIES \\ Dr. Douglas Guerra, \\ douglas.guerra@oswego.edu \\ MATHEMATICS \\ Dr. John Emert, \\ emert@bsu.edu \\ Dr. Jeffrey J. Boats, \\ boatsjj@udmercy.edu \\ Dr. J.D. Phillips, \\ jopbilli@nmu.edu \\ Dr. Dele Oluwade, \\ deleoluvade@yahoo.com \\ Dr. Christopher Baltus, \\ cbristopher.baltus@oswego.edu \\ Dr. Mark Baker, \\ mark.baker@oswego.edu
}




\section{SPECIAL THANKS}

AJUR is made possible through the volunteer efforts of our editorial team and assistance of our sponsors.

We thank our sponsors:

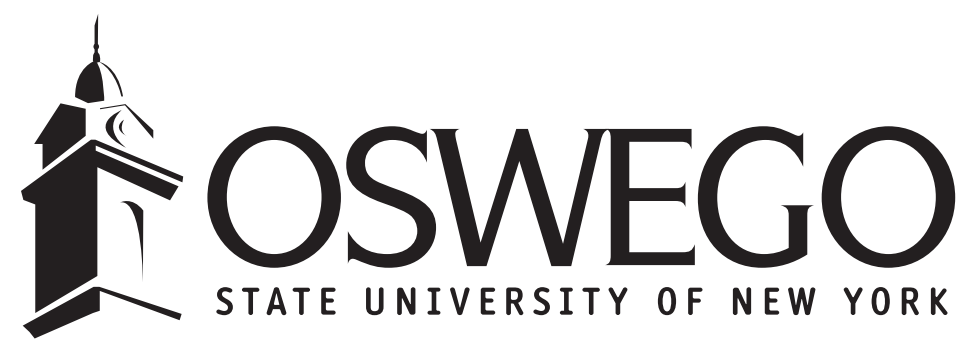

Please support undergraduate research

Request sponsorship information fromeditor@ajuronline.org 


\title{
Should high-top or low-top cleats be used when playing baseball?
}

\author{
Margo P. Woblfeil,, ${ }^{a}$ Cole R. Neary,,"\# Mitchell M. Klocke, Johnny G Mills, ${ }^{b}$ Rich C. Calvert, \& David S. Senchina ${ }^{b}$ \\ aBiochemistry, Cell, and Molecular Biology Program, Drake University, Des Moines, IA \\ ${ }^{b}$ Biology Department, Drake University, Des Moines, IA \\ 'Pharmacy Program, Drake University, Des Moines, IA \\ 'Athletics, Drake University, Des Moines, $L A$ \\ bttps:// doi.org/10.33697/ajur.2019.011 \\ Students:Margo.Wohlfeil@ucs.edu*,cole.neary@drake.edu,mitchell.klocke@indianoloa.k12.ia.us,john.mills@drake.edu \\ \# These authors are sharing joint first-authorship. \\ Mentors:david.senchina@drake.edu,rich.calvert@drake.edu
}

\begin{abstract}
There is a shortage of knowledge about how baseball cleat design may impact athletes. The purpose of this experiment was to determine if the height of the baseball cleat affected performance, perception of the cleat, and ankle range-of-motion. Thirteen subjects participated in the study, and each subject performed three drills (base-running, 5:10:5 "pro agility," and hitting) in both high-top and low-top baseball cleats. Performance time was measured for the first two drills. Perception of comfort, heaviness, shoe climate (heat), stability, and traction were measured for all three drills using $10-\mathrm{cm}$ visual analog scales (VAS). Ankle rangeof-motion (dorsiflexion, plantarflexion, eversion, and inversion) was measured using goniometry in both cleats, plus socks-only as baseline control. Shoe height did not significantly affect completion time in either the base-running drill (6.1 $\pm 0.1 \mathrm{sec}$. in high-top vs. $6.1 \pm 0.1 \mathrm{sec}$. in low-top) or pro-agility drill ( $4.8 \pm 0.1 \mathrm{sec}$. in high-top vs. $4.6 \pm 0.1 \mathrm{sec}$. in low-top). There were significant differences in perception of heat (6.4 in high-top vs. 4.6 in low-top; $p<.001)$, stability (6.9 in high-top vs. 5.1 in low-top; $p=.001$ ), and heaviness (6.0 in high-top vs. 4.1 in low-top; $p<.001)$, but not in perception of comfort (6.1 \pm 2.0 in high-top vs. $6.6 \pm 1.5$ in low top) or traction (7.3 \pm 2.0 in high-top vs. $7.4 \pm 1.5$ in low-top). There were significant differences between high-top and low-top cleats in plantarflexion ( $42.5^{\circ}$ in high-top vs. $47.5^{\circ}$ in low-top; $\left.p=.033\right)$, eversion $\left(7.9^{\circ}\right.$ in high-top vs. $11.3^{\circ}$ in low-top; $\left.p=.003\right)$, and inversion $\left(12.8^{\circ}\right.$ in high-top vs. $16.6^{\circ}$ in low-top; $\left.p=.044\right)$, but not dorsiflexion $\left(8.2^{\circ}\right.$ in high-top vs. $\left.10.9^{\circ}\right)$. For baseball players concerned about excessive ankle movements during play, these results suggest that using a high-top baseball cleat might reduce ankle range-of-motion without impairing performance or feeling burdensome.
\end{abstract}

\section{KEYWORDS}

Baseball; Cleats; High-Top; Low-Top; Perception; Performance; Range-of-Motion; Shoes

\section{INTRODUCTION}

Athletic shoes play a pivotal role in the comfort, protection, and motion of the athlete's ankle that can influence gross movement, performance, and potentially injury incidence. ${ }^{1}$ Athletic shoes are created specifically for the sport that is being played, ${ }^{2}$ the movements, and the amount of force applied to the foot during the sport. ${ }^{3}$ The height of the shoe is one of the main features that affect the athlete. Athletes participating in sports with more lateral movements tend to wear a high- or mid-top cleat, and athletes participating in sports with more linear movements will tend to wear a low-top cleat. ${ }^{2}$ In the context of this report, with high-top footwear the shoe collar (the upper rim of the shoe surrounding the ankle) completely covers the ankle malleoli ("ankle bones"), whereas with low-top footwear the shoe collar never comes up to the height of the ankle malleoli, and they remain exposed and with mid-top footwear the collar hits at the level of the malleoli.

Past studies have explored the effects of shoe height and its association with range-of-motion (ROM), performance, and ankle injuries during movements associated with basketball, ${ }^{4-11}$ American football, ${ }^{12-15}$ and volleyball. ${ }^{16}$ The seven studies that examined $\mathrm{ROM}^{4,8-11,13,16}$ all noted that the high-top shoes (or mid-top shoes in the volleyball study) reduced ankle rotation, extension/flexion, and/or eversion/inversion compared to their respective low-top counterparts. Regarding performance, five of the six studies that examined performance $\mathbf{8}^{8,911-13}$ showed that the high-top shoes did not affect performance compared to their respective low-top counterparts, whereas a sixth study ${ }^{6}$ reported that the high-top was associated with slower performance. The potential effect of high-top shoes on ankle injuries is less clear. One relevant study ${ }^{7}$ associated high-top shoes with reduced injuries, two additional studies ${ }^{5,14}$ showed no association between shoe height and injury, and one last study ${ }^{15}$ associated high-top shoes with increased injuries. Confounding factors such as concurrent use of taping/spatting and/or bracing, different player positions and anthropometrics, undocumented player changes in footwear during a game or season, or unaccounted out-of-sport 
physical activity patterns may explain why the injury results have been equivocal thus far. Sports medicine experts acknowledge these confounding factors and believe the preponderance of evidence still supports using high-top footwear as an ankle injury prevention measure. ${ }^{17,18}$

Less often studied is an athlete's perception of their footwear (for example, whether they find the footwear comfortable or stable), which will not only affect their footwear choices but also their performance. ${ }^{19,20}$ Only one published study ${ }^{13}$ (using football cleats) has previously looked at the association between shoe height and athlete perception of their footwear and reported that subjects found high-top cleats to be less comfortable, heavier, and more stable than low-top cleats. Since an athlete's perception of footwear often impacts their footwear selection (either initially or during the course of repeated exposure) and lack of comfort is often a predisposing factor for subsequent injury, ${ }^{21}$ it is important to consider the relationship between shoe design elements and the overall perceptual experience of the user. $^{17}$

Compared to the research done in basketball and football, there is little comparable data on baseball cleats. Baseball requires different movements than basketball or football, ${ }^{22}$ so it is unclear whether results from those sports' footwear translate to baseball footwear. Only three published studies were found to have examined baseball cleat design in field contexts. Two studies ${ }^{23,24}$ looked at only turf and soil variation and involved mechanical apparatuses instead of athletes, so they cannot speak towards athlete-associated outcomes. The third study ${ }^{25}$ reported that differences in the number of forefoot studs across various low-top baseball cleats did not influence subjects' performance or perception of the footwear. None of the studies examined high-top baseball footwear; however, high-top baseball cleats may be an essential consideration in the context of baseball ankle injury prevention given that a recent review of collegiate baseball injuries from 1988-2004 showed 8\% of all baseball injuries were ankle sprains. ${ }^{26}$ Information was not available as to what type of shoe the athletes were wearing, yet based on the literature reviewed previously, high-top cleats could conceivably reduce current ankle injury rates.

The purpose of this experiment was to determine if the height of the baseball cleat affected a player's performance, footwear perception, and ankle ROM by testing a high-top baseball cleat and a low-top baseball cleat from the same manufacturing series across identical baseball field drills. From previous research, it was hypothesized that: a) there would be no difference in performance outcomes between the two cleats; b) the high-top cleat would be perceived as heavier, less comfortable, more stable, and hotter than the low-top cleat (with no differences in perceived traction); and, c) the high-top cleat would reduce ankle ROM compared to the sock-only control and low-top cleat.

\section{METHODS AND PROCEDURES}

\section{Subject Characteristics}

The procedures were approved by the Drake University Institutional Review Board (2012-13013) before the study was carried out, and each subject gave written consent prior to participation. The subjects were thirteen college-aged (18-22 years old) males who were recreationally active (engaged in 2.5 hours or more of moderate-vigorous weekly physical activity). The subjects were able to complete the drills safely in a men's size 10,11.5, or 13-baseball cleat. College baseball players were not chosen for the study to avoid a priori biases.

\section{Shoes}

Two metal-studded baseball cleats were tested (Figure 1): a low-top (Under Armour Spine Metal; 1232831-002 Prod. Mo. October 2012) and a high-top (Under Armour Spine Highlight ST Metal; 1236979-001 Prod. Mo. November 2012; both from Under Armor, Baltimore, MD). The dimensions of the size 11.5 models were as follows: the low-top model was $13 \mathrm{~cm}$ high at the heel and near the tongue, and dropped to $10.5 \mathrm{~cm}$ between those features (i.e., in the malleolar notch); the high-top model was $20.5 \mathrm{~cm}$ tall at the front of the shoe and tapered to $17.5 \mathrm{~cm}$ by the heel, being tall enough to enclose the athletes' malleoli completely. The size 10 and 13 cleats were $\sim 1 \mathrm{~cm}$ shorter or taller (respectively) for each of those measurements. Both cleats had an identical stud configuration on the outsole and were new at the start of the experiment, making shaft height the only difference in the two cleats. Each subject was given new mid-calf socks ( $95 \%$ polyester, 5\% spandex; Body Glove International, Redondo Beach, CA) to wear during the experiment. Cleat presentation order was alternated.

\section{Procedure}

Anthropometrics were taken first, including height, weight, and body composition as determined by hand-to-foot bioelectrical impedance analysis [BIA] (BodyStat 1500; Cronkbourne, Douglas, Isle of Man, British Isles), followed by baseline measurements of ankle ROM (goniometry, including dorsiflexion, plantarflexion, eversion, and inversion) using a manual goniometer (HPMS Inc.; Windham, NH, USA), with subjects wearing socks but not shoes. The same experimenter performed all goniometry measurements throughout the entire study to ensure consistency across subjects. 


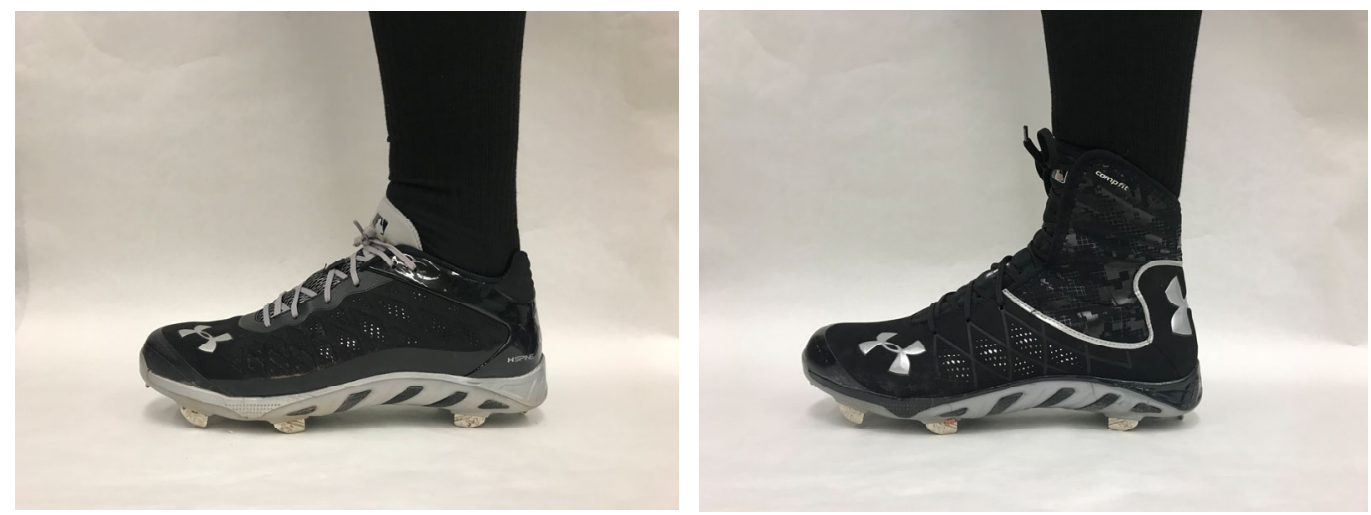

Figure 1. Low-top (left) and high-top (right) cleats used in this study.

Subjects were then given either the low-top or high-top cleat, and the goniometry was repeated in that cleat in a counterbalanced fashion, such that half of the subjects' first trial was with the low-top cleat and the other half of the subjects' first trial was with the high-top cleat. Each subject then completed a structured five-minute warm-up routine that emphasized dynamic stretching, including exercises such as the alternating high knee, raises while moving forward and alternating lunge with elbow touch to the knee while moving forward.

Next, the subject performed three baseball field drills outdoors at Drake University's Ron Buel softball field; these drills were chosen to engage the subjects with the cleats in different contexts. The first drill was a base-running drill. The subjects started at home plate and ran an all-out sprint around first base and through second base. Each subject was shown a standardized path to run the bases (banana loop turn at first base towards second instead of a square cut) so that all subjects used the same baserunning technique. Time was recorded using an electronic laser system (Brower Timing Systems LLC, Draper, UT, USA) when they crossed first base, and again when the subject ran through the second base. The second drill was a hitting drill and only tested perception. The subject was given ten baseballs to hit off a baseball tee into a net using a baseball bat. The third drill was a 5-10-5 ("pro agility") running drill. This drill was performed on the grass just outside the field using three disc cones each spaced 5 yards apart in a straight line. The subject started at the middle cone and was told to run to their right 5 yards, touch the cone, cut, and run back 10 yards to the leftmost cone, touch, cut, and then sprint through the original middle cone. Time was recorded manually. The subjects performed all three drills before switching into the second pair of cleats, and then the goniometry and three drills were repeated in the second cleat. Subjects performed all the drills for both cleats in a single session.

After each drill, the subjects were asked to rate their perception of the cleat's comfort, heaviness, foot climate (temperature), stability, and traction on separate 10-cm lines (visual analogue scales, VAS) with the left side of the line labeled as "The least (comfortable, heavy, hot, stable, traction) possible" and the right side of the line labeled as "The most (comfortable, heavy, hot, stable, traction) possible". Subjects made a vertical mark along the continuum to indicate their perception. VAS is scored by measuring from the left anchor to their vertical mark. A difference of $\sim 1 \mathrm{~cm}$ or higher in any comparison is typically considered functionally significant. ${ }^{20}$

\section{Statistics}

All statistics were computed in IBM SPSS Statistics 25. For goniometry, one-way ANOVA tests were run for each of the four measurements separately, where the footwear condition (socks only, low-top, or high-top) was the independent variable, and either dorsiflexion, plantarflexion, eversion, or inversion was the dependent variable. A p-value of $\leq 0.05$ was considered statistically significant. If a significant main effect was found, post hoc tests were computed using the least significant difference (LSD). For performance outcomes, paired samples t-tests comparing low-top to high-top were used, with four separate tests being run: one for each of the three base-running times (home plate to first base, first base to second base, and total route time) and one for the time to complete the 5-10-5 drill. After correcting the $\alpha$-level for the multiple comparisons, $\mathrm{p}$-values of $\leq 0.015$ were considered significant. For perceptual outcomes, paired samples t-tests comparing low-top to high-top were used, with five separate tests being run for each of the measured variables (comfort, heaviness, foot climate (temperature), stability, and traction). Each respective test included subject scores from all three exercises together. After correcting the $\alpha$-level for multiple comparisons, $\mathrm{p}$-values of $\leq 0.01$ were considered statistically significant. 


\section{RESULTS}

Thirteen subjects participated in the study with the following characteristics (mean \pm standard deviation): age $=19.5 \pm 1.8$, height $=177.8 \pm 5.2 \mathrm{~cm}$, weight $=79.2 \pm 12.1 \mathrm{~kg}$, and body fat percentage $=14.9 \pm 4.5$. Eight of the subjects wore a size $10 \mathrm{cleat}$, three of the subjects wore a size 11.5 cleat, and two subjects wore a size 13 cleat.

\section{Performance}

There were no significant differences found for performance between the two shoes during the two timed performance drills (all $p \geq 0.047$; Table 1).

\begin{tabular}{|c|c|c|c|c|}
\hline & & Low-Top & High-Top & $p$-value \\
\hline \multirow[t]{3}{*}{ Base-running Drill } & Home Plate to $1^{\text {st }}$ Base & $3.17 \pm 0.05$ & $3.15 \pm 0.04$ & 0.424 \\
\hline & $1^{\text {st }}$ Base to $2^{\text {nd }}$ Base & $2.97 \pm 0.06$ & $2.96 \pm 0.05$ & 0.955 \\
\hline & Total (Home Plate to $2^{\text {nd }}$ Base) & $6.14 \pm 0.10$ & $6.11 \pm 0.08$ & 0.628 \\
\hline 5-10-5 Drill & & $4.59 \pm 0.12$ & $4.81 \pm 0.13$ & 0.047 \\
\hline
\end{tabular}

Table 1. Time (in s) for the base-running and 5-10-5 drills. There were no significant comparisons between shoes because the A-level was 0.0125 after correcting Perception for multiple comparisons.

There were significant differences of footwear on perception for three of the five measures (heaviness, foot climate, and stability). Subjects perceived the high-top cleat as being significantly heavier $(p<.001)$, hotter $(p<.001)$, and more stable $(p=.001)$ than the low-top cleat (Figure 2). There was no significant difference found for the perceived comfort or traction between the two cleats (both $p \geq 0.235$; Table 2).
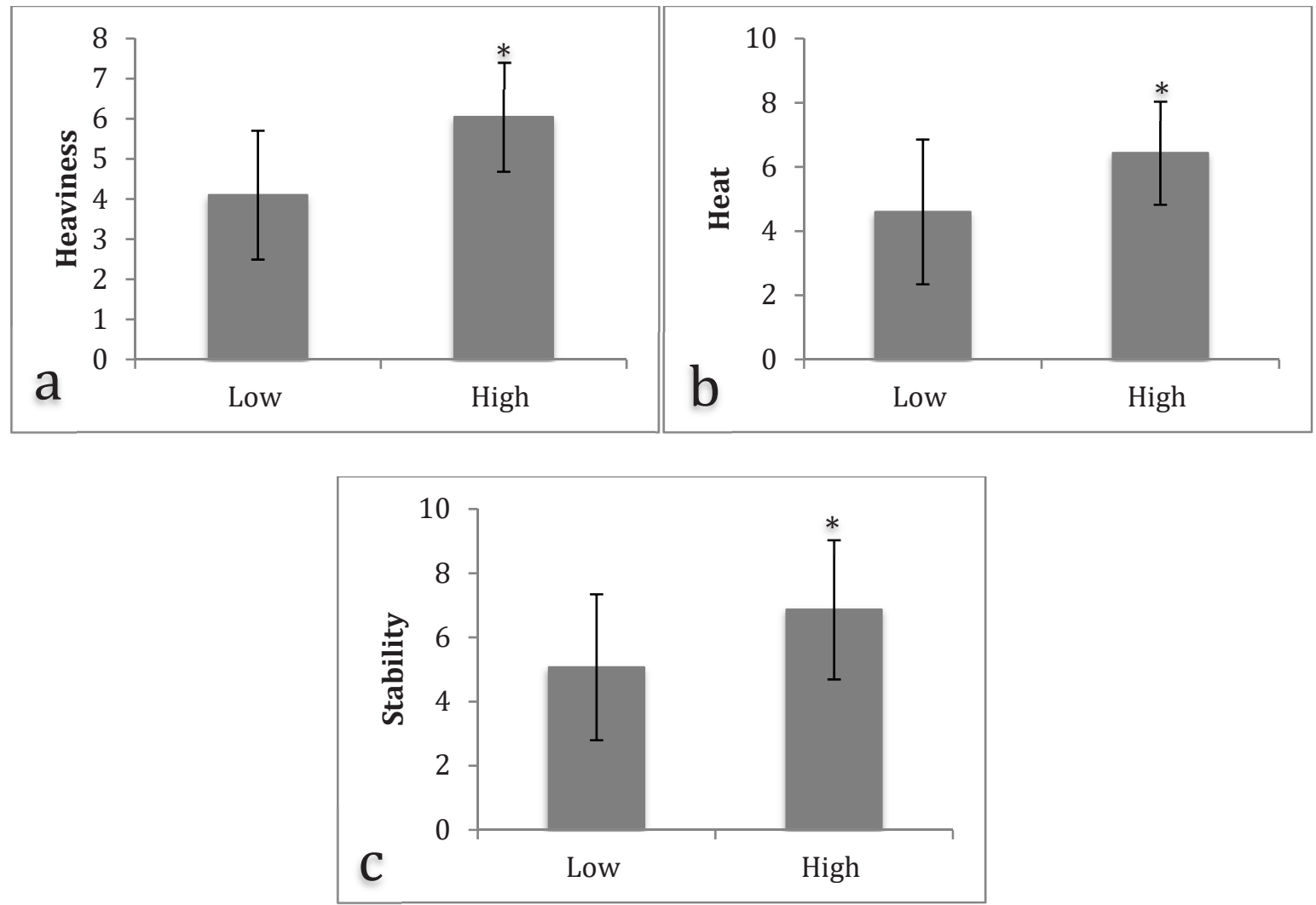

Figure 2. Perception of (a) heaviness, (b) foot climate (heat), and (c) stability in the low-top cleat vs. the high-top cleat. Values are in $\mathrm{cm}$ and based off a 10-cm scale, with higher values indicating more of a given perception. Asterisks mark significant differences between the high-top cleat and low-top cleat (all $p \leq 0.001$ ) 


\begin{tabular}{lcc}
\hline \hline & Comfort & Traction \\
\hline Low-Top & $6.6 \pm 1.5$ & $7.4 \pm 1.5$ \\
High-Top & $6.1 \pm 2.0$ & $7.3 \pm 2.0$ \\
\hline \hline
\end{tabular}

Table 2. Perception of comfort and traction in the low-top cleat vs. the high-top cleat. Values are in $\mathrm{cm}$ and based off a 10 -cm scale, with higher values indicating more of a given perception. There were no significant comparisons between shoes for comfort or traction (both $p \geq 0.235$ ).

\section{Goniometry}

There were significant differences for all four ankle motions (dorsiflexion $p=.037$, plantarflexion $p<.001$, eversion $p<.001$, inversion $p=.008$ ). Post hoc tests were then used to examine possible pairwise differences among the three footwear conditions. Dorsiflexion was significantly higher in the sock-only condition compared to the high-top cleat $(p=.012)$, but there were no significant differences between the sock-only condition and the low-top cleat, nor between the low-top cleat and the high-top cleat (both $p \geq 0.092$; Figure 3a). For plantarflexion, there were significant differences between all footwear comparisons such that plantarflexion was higher in the sock-only condition compared to the low-top cleat $(p=.001)$ and the high-top cleat $(p<.001)$, and greater in the low-top cleat compared to the high-top cleat $(p=.033$; Figure $3 \mathbf{b})$. For eversion, there were significant differences between all footwear comparisons such that eversion was greater in the sock-only condition compared to the low-top cleat $(p=.042)$ and the high-top cleat $(p<.001)$, and greater in the low-top cleat compare to the high-top cleat $(p=.003$; Figure 3c). Inversion was significantly less in the high-top cleat compared to both the sock-only condition $(p=.002)$ and low-top cleat $(p=.044)$ but was not different between the sock-only condition and low-top cleat $(p=0.249$; Figure 3d).
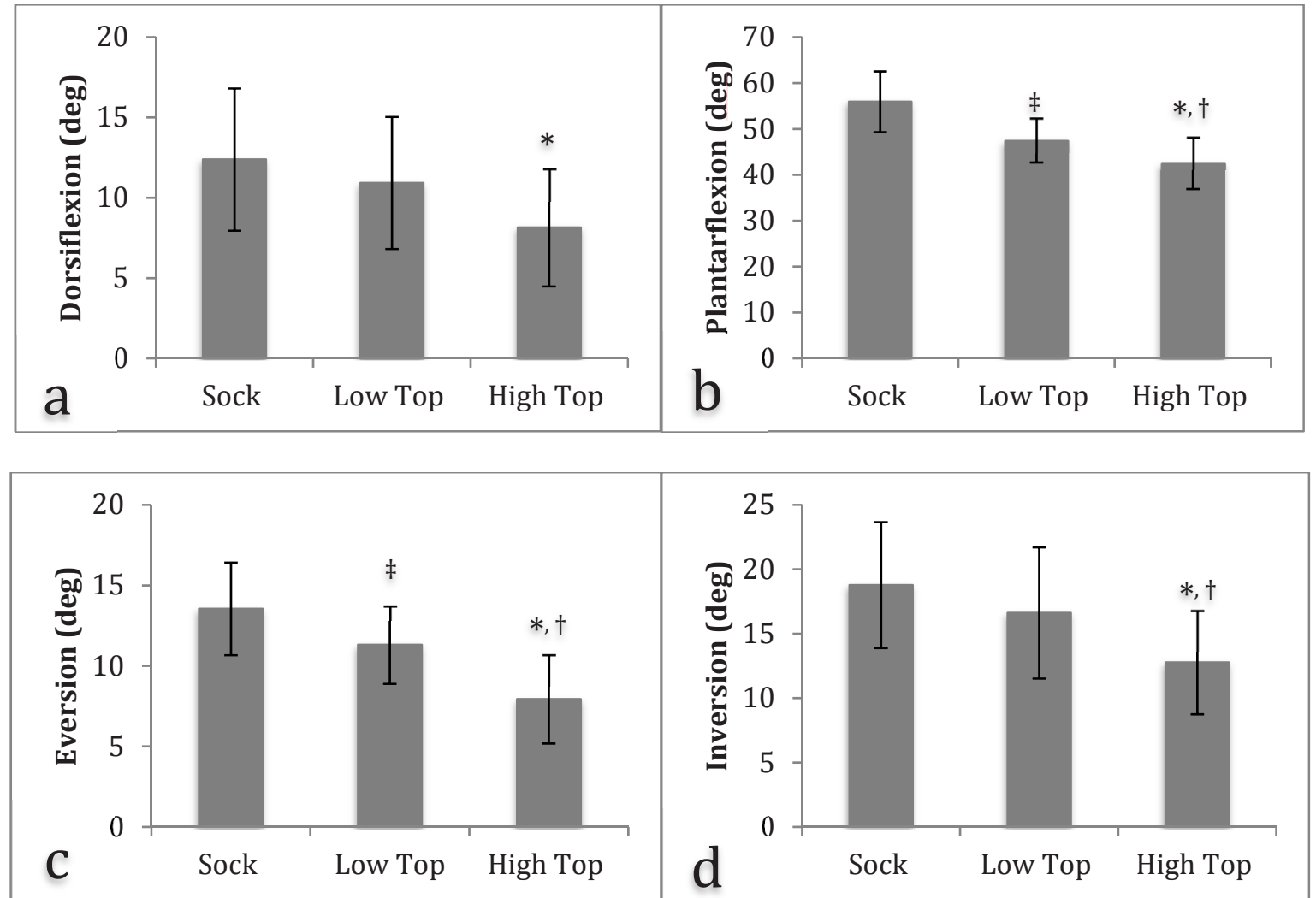

Figure 3. Range-of-motion (expressed as ${ }^{\circ}$ ) for (a) dorsiflexion, (b) plantarflexion, (c) eversion, and (d) inversion in the sock-only condition (baseline) and while wearing the low-top cleat and the high-top cleat. Asterisks mark a significant difference between high-top and sock. Daggers mark a significant difference between high-top and low-top. Double daggers indicate a significant difference between low-top and sock. See preceding text for individual $p$-values for all possible comparisons. 


\section{DISCUSSION}

\section{Performance}

Hypothesis A was that there would be no statistically significant performance differences when the baseball drills were performed in low-top compared to high-top baseball cleats. Results supported that hypothesis (Table 1). This is the first known study to investigate how baseball shoe height could impact performance in a baseball field setting.

Previous studies on true high-tops and athletic performance have been limited to basketball and football. Both of the football studies $^{12,13}$ (spaced forty years apart and involving football cleats of very different designs and materials respective to their time periods) tested low-top to high-top football cleats in football-specific field drills; both found insignificant differences in performance time, and concluded that the shaft height of the football cleat did not affect athlete performance. Likewise, three of four contemporary basketball shoe studies ${ }^{8,9,11}$ that utilized basketball-specific court drills found insignificant differences in performance time and reached a similar conclusion. A lone basketball study ${ }^{6}$ found that their high-top shoe model decreased obstacle course times and vertical jump height, but it is unclear why that particular experiment differed from the others. Results from the present study were thus similar to the majority ${ }^{8,9,11-13}$ of those previous basketball and football studies in showing that high-top shoes did not appear to deleteriously affect performance.

Some high-top athletic shoes have a consistent or mostly consistent collar height around the circumference of its shaft (such that the collar height is always above the height of the ankle malleoli, regardless of whether it is directly above the malleoli or elsewhere such as above the Achilles tendon). Whereas other high-top athletic shoes have a variable collar height (such that the collar is always higher around the malleoli, but then noticeably dips around the Achilles tendon such that it is then at or below the height of the malleoli). Current high-top baseball and football cleats tend to be of the former variety, whereas current high-top basketball shoes are more often of the latter variety. Based on the pictures provided in the publications or historical context, the football and baseball cleats used in the aforementioned studies all had consistent collar height, whereas the basketball shoes used in the aforementioned studies all had variable collar height. The collective findings may suggest that consistent high collar height around the entire circumference of the ankle does not harm performance (i.e., there may be no practical benefit of having a lower collar height at the Achilles tendon, or stated another way, the additional material around the Achilles tendon may not impede its movement). However, it should be noted that field and court surfaces present very different conditions for athletic performance, so a direct comparison between the two surfaces would be necessary to investigate that possibility.

\section{Perception}

Hypothesis B was that the high-top cleat would be perceived to be less comfortable, hotter, more stable, and heavier than the low-top cleat (with no differences in perceived traction). The data for heaviness, foot climate, and stability (Figure 2) were consistent with the hypothesis; however, the data for perceived comfort (Table 2) opposed our hypothesis because subjects reported no perceived differences between the high-top and low-top baseball cleats. As mentioned previously in "Methods and Procedures," other research teams have established that a difference of $\sim 1 \mathrm{~cm}$ or greater indicates a functionally relevant difference between two perceptual scores. Figure 2 shows that the threshold was exceeded for all three statistically significant comparisons.

The lone previously published study ${ }^{13}$ to look at shoe height and athlete footwear perception was in the context of football cleats and football-specific field drills, and it is the only point of comparison for the present perceptual data. The previous study reported that subjects perceived the high-top cleat to be less comfortable, heavier, and more stable than the low-top cleat (it did not assess foot climate or traction); thus, results are mostly consistent between the previous and present studies. One important difference between the football cleat used in the previous study and the baseball cleat used in the present study is the material that made up the shoe shaft — the football cleat had very thick padding throughout its circumference, whereas the baseball cleat had thinner fabric-like material. Since football is a contact sport and most high-top football cleats are used by linemen, the additional padding serves a protective function. Differences in the shoe shaft composition may explain why there were differences in comfort perception between the two studies; however, more studies will be needed to investigate that possibility.

\section{Range-of-motion}

Hypothesis $\mathrm{C}$ was that the high-top baseball cleat would significantly reduce the ankle ROM compared to the sock control and low-top cleat. The first part of this hypothesis concerned the high-top cleat $v$ s. sock-only control, and in those comparisons, results indicated the high-top cleat significantly reduced all four motions (dorsiflexion, plantarflexion, eversion, and inversion) compared to the sock control (Figure 3). The second part of this hypothesis concerned the high-top cleat vs. the low-top cleat. Plantarflexion, inversion, and eversion were reduced in the high-top cleat compared to the low-top cleat (Figures 3b, 3c, and 3d), but there were no differences in dorsiflexion between the two cleat models (Figure 3a). Overall, these results support the hypothesis that the high-top cleat significantly reduced ankle ROM. 
Sport-associated ankle sprains are typically caused by excessive inversion (as opposed to eversion) movements. ${ }^{27-29}$ If high-top athletic footwear is to reduce ankle sprain injury rates in athletes, then such footwear should limit inversion. Both the present study (Figure 3) and all of the previously mentioned basketball and football shoe studies that measured inversion $4,9,10,13$ found the high-top shoe reduced inversion compared to the low-top shoe. Given that these studies collectively utilized a variety of techniques across a variety of sport-specific shoe models and athletes, and all yielded the same conclusion, high-top athletic footwear is likely efficacious in reducing inversion.

Interpreting the dorsiflexion/plantarflexion results requires more nuance. Inversion sprains typically occur when the foot is simultaneously in a forced plantarflexed condition. ${ }^{29}$ Poor dorsiflexion strength and/or low dorsiflexion ROM (e.g., as would be needed to oppose a force plantarflexed position) has been associated with higher inversion sprains in some prospective studies of college-aged athletes ${ }^{30,31}$ but not in others. ${ }^{32,33}$ In the present study, the high-top cleat limited plantarflexion (but not dorsiflexion) compared to the low-top cleat (Figure 3). If one errs on the side of caution and assumes excessive plantarflexion and/or dorsiflexion deficiencies may contribute to the occurrence or severity of inversion ankle sprains, then it is beneficial for high-tops to limit plantarflexion but not dorsiflexion. Two of the previously mentioned high-top studies also looked at dorsiflexion/plantarflexion movements. The one basketball study that did $\mathrm{so}^{11}$ found that the high-top shoe significantly reduced plantarflexion and had various effects on dorsiflexion compared to the low-top shoe, whereas the one football study that did so ${ }^{13}$ found that the high-top shoe reduced dorsiflexion (but not plantarflexion) compared to the low-top shoe. Since there is only one study each from baseball, basketball, and football to compare and their collective results appear equivocal, it is likely premature to form any conclusions about high-top athletic footwear generally in terms of this motion or whether high-top cleats might be protective in terms of baseball athletes recovering from ankle injuries.

\section{Limitations}

Several limitations exist in this study. First, recreationally active students were used instead of baseball athletes to avoid any biases that baseball athletes may have towards one cleat type or another, which could have influenced study outcomes (based on their past playing experiences); thus, subjects in the present study may not have run the drills with the same amount of speed and force generated by a baseball player. Use of recreationally active individuals instead of competitive university athletes, whether to avoid a priori bias or for convenience sampling, is typical in footwear research. Of the twenty direct experimental or prospective studies cited in this publication ${ }^{4-16,19,20,25,30-33}$, eleven utilized recreationally active individuals (whether to avoid bias or as convenience sampling), eight utilized competitive university athletes, and one was indeterminate in regards to subject status. Second, only one baseball cleat design was used in the present study. In baseball, there are many different cleat manufacturers, and within each, there are many different styles of cleats. Therefore, these findings will not likely apply to all styles of baseball cleats. Third, this field study did not have access to technology that could have improved precision, or allowed for measuring of joint angles (e.g., ankle, knee, hip) during the field drills. This data could show ankle ROM in real time during the specific drills, or how the shoe height affected the entire kinetic chain. Goniometry in this study was limited to voluntary movements while seated, and not actual game movements; further, the baseline goniometry was performed before warm-up to avoid subject attentional and physical fatigue (logistically allowing us to separate each round of goniometry by approximately 20 minutes). Fourth, this was an acute experiment that did not simulate a full game or practice experience, and results may be different if the athlete was tested throughout a season.

\section{CONCLUSIONS}

There were three significant findings with regards to the specific baseball cleat model used in this study: (1) the high-top baseball cleat did not negatively influence baseball drill performance compared to the low-top cleat; (2) the high-top baseball cleat is perceived as equally comfortable but more stable than the low-top baseball cleat; and (3) the high-top baseball cleat reduces eversion, inversion, and plantarflexion compared to the low-top cleat. Athletes or coaches considering high-top baseball cleats may be able to use this knowledge in selecting footwear, and the findings may allay concerns baseball athletes have about high-top cleats slowing their movements. This study is novel because it is the first to examine the role of baseball cleat shaft height in the context of athlete performance, footwear perception, or ankle ROM, and is only the second baseball footwear study directly involving athletes.

In the future, it could be beneficial to repeat this study with college baseball players or different models/brands of baseball cleats or to perform a longitudinal study looking at the effects of the cleats over several practices or games. Baseball athletes concerned about ankle stability frequently use other external ankle support devices such as braces or taping instead of or in addition to hightop footwear, so future studies could also look at the effects of those items combined or separately to see if there are advantages of one method versus another or potential additive effects. Studying other design features of the cleat like its material, stud configuration, mass, and sole properties may provide additional insights into optimal baseball footwear design. 


\section{Acknowledgments}

We thank our 13 subjects who volunteered their time to participate in this study. Also, we thank you Drake Athletics for allowing our research team to use Ron Buel field for the field drills.

\section{REFERENCES}

1. Barnes, R. A., and Smith, P. D. (1994) The role of footwear in minimizing lower limb injury, J Sports Sci 12(4), $341-353$. https:/ / doi.org/ 10.1080/02640419408732180

2. Caselli, M. A. (2006) Selecting the proper athletic shoe, Podiatry Manag 25(8), 147-156.

3. Shorten, M. (2004) Running Shoe Design: Protection and Performance, in Marathon Medicine (Pedoe, D.T., Ed.) 159-169, Royal Society of Medicine Press Limited: London, UK.

4. Ashton-Miller, J. A., Ottaviani, R. A., Hutchinson, C., and Wojtys, E. M. (1996) What best protects the inverted weightbearing ankle against further inversion? Evertor muscle strength compares favorably with shoe height, athletic tape, and three orthoses Am J Sports Med 24(6), 800-809. https:/ / doi.org/10.1177/036354659602400616

5. Barrett, J. R., Tanji, J. L., Drake, C., Fuller, D., Kawasaki, R. I., and Fenton, R. M. (1993) High- versus low-top shoes for the prevention of ankle sprains in basketball players. A prospective randomized study, Am J Sports Med 21(4), 582-585. bttps:/ / doi.org/ 10.1177/036354659302100416

6. Brizuela, G., Llana, S., Ferrandis, R., and García-Belenguer, A. C. (1997) The influence of basketball shoes with increased ankle support on shock attenuation and performance in running and jumping, J Sports Sci 15(5), 505-515. bttps://doi.org/10.1080/026404197367146

7. Garrick, J. G., and Requa, R. K. (1973) Role of external support in the prevention of ankle sprains, Med Sci Sports 5(3), 200203. https:// doi.org/10.1249/00005768-197323000-00020

8. Lam, G. W. K., Park, E. J., Lee, K.-K., and Cheung, J. T.-M. (2015) Shoe collar height effect on athletic performance, ankle joint kinematics and kinetics during unanticipated maximum-effort side-cutting performance, J Sports Sci 33(16), 1738-1749. bttps:// doi.org/10.1080/02640414.2015.1011206

9. Liu, H., Wu, Z., and Lam, W.-K. (2017) Collar height and heel counter-stiffness for ankle stability and athletic performance in basketball, Res Sports Med 25(2), 209-218. bttps:/ / doi.org/10.1080/15438627.2017.1282352

10. Ottaviani, R. A., Ashton-Miller, J. A., Kothari, S. U., and Wojtys, E. M. (1995) Basketball shoe height and the maximal muscular resistance to applied ankle inversion and eversion moments, Am J Sports Med 23(4), 418-423. bttps://doi.org/10.1177/036354659502300408

11. Yang, Y., Fang, Y., Zhang, X., He, J., and Fu, W. (2017) Does shoe collar height influence ankle joint kinematics and kinetics in sagittal plane maneuvers?, J Sports Sci Med 16(4), 543-550.

12. Bauer, H. (1970) The effect of high-top and low-cut football shoes on speed and agility, Athl J 50, 74-75,86-87.

13. Daack, C. W., Senchina, D. S. (2014) A field study of low-top vs. mid-top vs. high-top American football cleats, Sports 2(4), 85-98. https:// doi.org/10.3390/sports2040085

14. Iacovelli, J. N., Yang, J., Thomas, G., Wu, H., Schiltz, T., and Foster, D. T. (2013) The effect of field condition and shoe type on lower extremity injuries in American football, BrJ Sports Med 4(12), 789-793. https:// doi.org/10.1136/ bjsports-2012-092113

15. Rovere, G. D., Clarke, T. J., Yates, C. S., and Burley, K. (1988) Retrospective comparison of taping and ankle stabilizers in preventing ankle injuries, Am J Sports Med 16(3), 228-233. https:/ / doi.org/10.1177/036354658801600305

16. Cronin, A. (2001) Effect of Selected Ankle Supports on Resistance to Inversion Force and Range of Motion. B.Sc., University of Ottawa: Ottawa, Ontario, Canada.

17. Petrov, O., Blocher, K., Bradbury, R. L., Saxena, A., and Toy, M. L. (1988) Footwear and ankle stability in the basketball player, Clin Podiatr. Med Surg 5(2), 275-290.

18. Ryan, A. J. (1986) Ankle sprains: A roundtable, Phys Sportsmed 14(2), 101-118.

19. Lam, W. K., Sterzing, T., and Cheung, J. T.-M. (2011) Reliability of a basketball specific testing protocol for footwear fit and comfort perception, Footwear Sci, 3(3), 151-158. https:// doi.org/10.1080/19424280.2011.630680

20. Mills, K., Blanch, P., and Vicenzino, B. (2010) Identifying clinically meaningful tools for measuring comfort perception of footwear, Med Sci Sports Exerc 42(10), 1966-1971. https:/ / doi.org/10.1249/MSS.0b013e3181 dbacc8

21. Mündermann, A., Stefanyshyn, D. J., and Nigg, B. M. (2001) Relationship between footwear comfort of shoe inserts and anthropometric and sensory factors, Med Sci Sports Exerc 33(11), 1939-1945. https://doi.org/10.1097/00005768-20011100000021

22. Dutra, T. (2010) Baseball and Softball, in Athletic Footwear and Orthoses in Sports Medicine (Werd, M. B., and Knight, E. L., Eds.) 303-306, Springer, New York.

23. Brosnan, J. T., McNitt, A. S., and Serensits, T. J. (2009) Effects of varying surface characteristics on the hardness and traction of baseball field playing surfaces, Int Turfgrass Soc Res J 11, 1053-1066.

24. Goodall, S. A., Guillard, K., Dest, W. A., and Demars, K. R. (2005) Ball response and traction of skinned infields amended with calcined clay at varying soil moisture contents, Int Turfgrass Soc Res J 10, 1085-1093. 
25. Gibson, D. G., Calvert, R. C., and Senchina, D. S. (2014) Does the number of spikes in the baseball cleat forefoot impact on performance or perception of traction?, J Athl Med 2(2), 53-65.

26. Hootman, J. M., Dick, R., and Agel, J. (2007) Epidemiology of collegiate injuries for 15 sports: Summary and recommendations for injury prevention initiatives, $J$ Athl Train 42(2), 311-319.

27. Barrett, J., and Bilisko, T. (1995) The role of shoes in the prevention of ankle sprains, Sports Med, 20(4), 277-280. bttps:/ / doi.org/ 10.2165/00007256-199520040-00005

28. Garrick, J. G. (1977) The frequency of injury, mechanism of injury, and epidemiology of ankle sprains, Am J Sports Med 5(6), 241-242. https:// doi.org/10.1177/036354657700500606

29. Verhagen, E. A., van Mechelen, W., and de Vente, W. (2000) The effect of preventive measures on the incidence of ankle sprains, Clin J Sport Med 10(4), 291-296. https:/ / doi.org/10.1097/00042752-200010000-00012

30. Baumhauer, J. F., Alosa, D. M., Renström, A. F., Trevino, S., and Beynnon, B. A (1995) Prospective study of ankle injury risk factors, Am J Sports Med 23(5), 564-570. https:// doi.org/10.1177/036354659502300508

31. Willems, T. M., Witvrouw, E., Delbaere, K., Mahieu, N., De Bourdeaudhuij, L., and De Clercq, D. (2005) Intrinsic risk factors for inversion ankle sprains in male subjects: A prospective study, Am J Sports Med 33(3), 415-423. https:// doi.org/10.1177/0363546504268137

32. Beynnon, B. D., Renström, P. A., Alosa, D. M., Baumhauer, J. F., and Vacek, P. M. (2001) Ankle ligament injury risk factors: A prospective study of college athletes, J Orthop Res 19(2), 213-220. https:/ / doi.org/ 10.1016/ S0736-0266(00)90004-4

33. Payne, K. A., Berg, K., and Latin, R. W. (1997) Ankle injuries and ankle strength, flexibility, and proprioception in college basketball players, J Athl Train 32(3), 221-225.

\section{ABOUT STUDENT AUTHORS}

Margo Wohlfeil graduated from Drake University in December of 2017 with a B.A. in Biochemistry, Cell, and Molecular Biology. She is currently working as a Junior Research Specialist in a pediatric oncology lab at the University of California, San Francisco.

Cole Neary graduated from Drake University in December of 2018 with a B.S. in Biology. He is now working for the Drake University Athletic Department as a development coordinator.

Mitchell Klocke currently lives in Indianola, IA. He graduated from Drake University in May of 2018 with a B.A. in Biology, as well as a B.S.E in Secondary Education. Currently, he teaches 7th-grade science at Indianola Middle School in Indianola, IA.

Johnny Mills will graduate from the College of Pharmacy and Health Science at Drake University in May of 2021 with his Pharm.D. He plans to work in the pharmaceutical industry or community setting after his education.

\section{PRESS SUMMARY}

Thirteen recreationally active males completed a series of drills in high-top and low-top baseball cleats and performance outcomes, ankle range-of-motion (ROM), and athlete perception were all recorded. Shoe height had no impact on performance; however, the high-top cleat limited ankle ROM and was perceived as heavier, stable, and hotter. The results suggest that high-top baseball cleats may be a viable solution for players concerned about ankle stability, without negatively impacting performance. 


\title{
Transcription of nanos-1 in Zebrafish Embryos is not Affected by Bisphenol A: Evaluated Using Quantitative Real-Time PCR
}

\author{
Bridget Babich, George Roba, Siti Sarah Safura*, Kevin Callaban, \& Edward Freeman \\ Department of Biology, St. John Fisher College, NY \\ bttps://doi.org/10.33697/ajur.2019.012
}

Students: bnb08764@sjfc.edu,gr00103@sjfc.edu,sss03429@sifc.edu*

Mentors:efreeman@sjfc.edu and kcallahan@sifc.edu

\begin{abstract}
The presence of primordial germ cells (PGCs) is crucial for proper gonad formation in zebrafish (Danio rerio). The many aspects of PGC migration that allow these cells to reach the proper location at the gonadal ridge include receptors, ligands, germ plasm components, and internal maintenance of PGCs. Any one of these factors could be affected by endocrine-disrupting chemicals (EDCs), which have been shown to alter the directed migration of these cells during early embryonic development. Based on recent research wherein the EDC bisphenol A (BPA) inhibited normal PGC migration, we have used the same dose of BPA to determine the impact of BPA on a gene central to proper germ cell migration. Zebrafish embryos were exposed to BPA, and the levels of the target gene nanos-1 were analyzed using quantitative real-time PCR (q-PCR). The target gene nanos-1 is a critically important germplasm component that allows for survival and proper migration of PGCs. The q-PCR results showed that BPA did not affect the transcription level of nanos-1 in zebrafish embryos.
\end{abstract}

\section{KEYWORDS}

Zebrafish; Zebrafish Embryos; nanos-1; Primordial Germ Cells; PGC Migration; Gonad Development; Endocrine-Disrupting Chemicals; Bisphenol A; Sex Determination

\section{INTRODUCTION}

Primordial germ cells (PGCs) are important for proper gonad development in both vertebrates and invertebrates and are wellstudied in common model organisms such as Drosophila, mice, zebrafish, and Xenopus., ${ }^{1,2}$ PGCs are originally located in the primary ectoderm and must migrate to the gonadal ridge, where they later differentiate into gametes. In humans, PGC migration occurs between embryonic weeks four and six. ${ }^{3}$ In mice, PGC migration begins on embryonic day eight, and the cells reach the gonadal ridge around embryonic day ten. ${ }^{4}$ In zebrafish, PGCs reach the gonadal ridge by 24 hours post fertilization (hpf) after using amoeboid movements to travel along the gonadal pathway to reach their final location at the gonadal ridge. ${ }^{1,5}$ However, when PGC migration is disrupted, the cells are unable to reach the gonad region, and improper gonad development occurs. ${ }^{6}$ Normal PGC migration requires several interrelated mechanisms. The first mechanism is attraction and repulsion of the cells via chemical cues from indicator cells, which bind to receptors on PGCs. ${ }^{7}$ Another mechanism that controls migration is the composition of germ plasm components dictated by maternal effect genes, which are active early in embryogenesis and allow for proper migration. ${ }^{8}$ Maternal effect genes are repressed when zygotic genes later become active. ${ }^{8,9}$ Both of these processes have been shown to play a crucial role in zebrafish PGC migration. ${ }^{1,9}$

Zebrafish are an ideal model organism for the study of PGC migration because embryos are generated in large numbers, develop quickly, and are transparent during the first 24 hpf. $^{6}$ In addition, much is known about the PGC migration pathway. As stated above, PGCs follow the gonadal pathway and are led by attractants and repellents. One well-studied chemoattractant is SDF-1a. This attractant is secreted by somatic cells and binds to the CXCR4b receptor on PGCs. ${ }^{1}$ Another receptor, CXCR7, controls the distribution of SDF-1a, which polarizes PGCs and directs their migration toward attractants. ${ }^{7}$ Along with these receptor and signal proteins that control migration, many germplasm genes also play important roles in proper migration. nanos-1 is a germplasm component that serves to promote PGC migration and also ensures the survival of these cells. ${ }^{10}$ Because of its essential role in the PGC migration pathway, nanos-1 is highly conserved across species and is initially a maternal effect gene. ${ }^{10}$ This gene product is primarily present during PGC migration and again during PGC incorporation into the gonad during the first five days of development. ${ }^{10}$ In a 2001 study, when nanos- 1 was knocked-down using a morpholino, PGCs did not develop normally and were unable to migrate to the gonadal ridge. ${ }^{10}$ Rather, when the concentration of the nanos- 1 protein was decreased or diminished, PGCs migrated to the somites and head region instead, where they only survived for a couple of days. ${ }^{10}$ These results illustrate the importance of nanos-1 from both a migration and maintenance perspective of PGCs in a vertebrate model organism. 
Although morpholinos are an important tool when studying the function of specific genes, they are not useful when looking at changes in gene expression caused by factors in the natural environment. Specifically, the effects of pollutants in the environment cannot directly be studied using morpholinos. Many pollutants are used as pesticides and herbicides and have adverse effects on non-target organisms, including zebrafish. Plasticizers are a type of pollutant, and collectively, these and other chemicals fall under the broad category of endocrine-disrupting chemicals (EDCs). EDCs may mimic hormones and have the ability to disrupt a variety of hormone-regulated processes. ${ }^{11}$ In a 2001 study, the EDCs endosulfan and nonylphenol were studied to evaluate their impact on PGC migration. ${ }^{11}$ The results of this study showed that when zebrafish embryos were exposed to endosulfan, there was a decreased number of PGCs and the PGCs that were present were redistributed in the embryo. ${ }^{11}$ Similar to endosulfan, nonylphenol exposure resulted in a redistribution effect, and both EDCs evaluated in this study had estrogenic effects. ${ }^{11}$ Another study evaluated the effects of deltamethrin — a pesticide that is used to kill parasites of fish but in high doses can cause metabolic disorders as it accumulates in fish tissues. ${ }^{6}$ The results showed that deltamethrin exposure causes PGCs to have structural deformities and slowed movement through the migration pathway. ${ }^{6}$ High dose exposure to deltamethrin caused PGCs to have significant morphological changes that eliminated migration. ${ }^{6}$

Another relevant EDC is Bisphenol A (BPA), a common organic chemical found in resins and many plastics. BPA has been linked to many human diseases, such as breast cancer, diabetes, and various cardiovascular, chronic respiratory, kidney, and reproductive diseases. ${ }^{12} \mathrm{BPA}$ is commonly found in the environment, especially in bodies of water due to its ubiquitous nature and common use by manufacturers. ${ }^{13}$ Similar to endosulfan and nonylphenol, BPA mimics estrogen and disrupts normal gonad formation. ${ }^{14} \mathrm{BPA}$ is therefore considered an endocrine-disrupting chemical due to its strong estrogenic mimicry. ${ }^{14} \mathrm{~A} 2017$ study found that exposure to concentrations of BPA as low as $0.001 \mu \mathrm{M}$ during development can severely impact zebrafish reproductive health, based on analysis of morphological traits post-exposure. ${ }^{15}$ The amount of BPA in the environment varies widely among geographical regions. With some areas near water waste treatment plants (WWTP) found to range from not detectable to $1.6 \mu \mathrm{M}$ and other areas reporting maximums of $36.8 \mu \mathrm{M}$ and $45.1 \mu \mathrm{M} .16,17,18$ Marine and coastal water was also measured and showed that concentrations of BPA ranged from $0.00017 \mu \mathrm{M}$ to $0.00085 \mu \mathrm{M}$, and portable tap water was found to have a maximum BPA concentration of $5.7 \mu \mathrm{M} .{ }^{16} \mathrm{BPA}$ tends to exhibit a nonmonotonic dose-response curve-meaning that there is a nonlinear relationship between effects and doses. ${ }^{19}$ This response pattern indicates that smaller, trace BPA concentrations in aquatic environments can still have adverse effects on aquatic organisms.

In a 2013 study, zebrafish embryos were exposed to two doses of BPA, $17.5 \mu \mathrm{M}$ and $35 \mu \mathrm{M}$, during the first 24 hours of development (note that these doses were initially reported in $\mathrm{mg} / \mathrm{L}$ units but were converted here for consistency with other published work). ${ }^{5}$ In situ hybridization was used to screen for the vasa gene, a marker for PGCs, to determine how BPA affected PGC migration. The results illustrated that both doses of BPA cause PGCs to migrate to ectopic locations. ${ }^{5}$ Many of the PGCs were found anterior to the gonadal region. ${ }^{5}$ All four of the above EDCs affected PGCs in similar ways: each chemical prevented PGCs from reaching the gonadal ridge. Thus, through in situ hybridization and histological profiles, studies have shown that PGCs migrate improperly and have morphological changes in the presence of certain EDCs. However, the mechanism(s) that underlie these changes are still unknown.

When looking at migration patterns of PGCs, there are three important things to consider: the signal being sent to the cells, the receptors on the cells, and the germplasm components. Each of these could potentially be affected by EDC exposure-however, none have been studied. Therefore, in this study, zebrafish embryos were exposed to the lower of the two published doses of BPA that were shown to impair PGC migration, $17.5 \mu \mathrm{M}$ (note that we confirmed that exposure to $17.5 \mu \mathrm{M}$ BPA disrupted PGC migration in our laboratory setting, data not shown). Specifically, we sought to determine if a critical gene associated with PGC migration and maintenance (nanos-1) was impacted when migration was impaired due to BPA exposure. Using q-PCR, nanos-1 was analyzed to determine $\Delta \Delta \mathrm{Ct}$ against housekeeping genes efla and 18 s. A 2008 study found that out of eight commonly used housekeeping genes, ef1 $a$ and $18 s$ were among the most stably expressed of those eight genes, which is why we have selected them as housekeeping genes. ${ }^{20}$ As indicated above, nanos- 1 is a germplasm component found in PGCs and, based on its critical importance to normal PGC migration, is a likely target to explain impaired PGC migration with EDC exposure.

\section{METHODS AND PROCEDURES}

\section{Fish maintenance}

Zebrafish were purchased at local pet stores in Rochester, NY and allowed to acclimate to the housing system for at least two weeks. Experimentation was reviewed and approved by the Institutional Animal Care and Use Committee at St. John Fisher College (IACUC Protocol \#61). Male and female zebrafish were kept together in tanks that had a constant flow of system water. System water was maintained at $27-29^{\circ} \mathrm{C}$ with a salinity between $1400-1600$ microsiemens, which was checked every other day. The $\mathrm{pH}$ level was kept between 6.9-7.4 and was measured twice per week. The fish were kept on a 14-hour light/10-hour dark 
schedule and fed twice daily. The fish were fed with live brine shrimp (Utah Red Shrimp from Artemac L.L.C) and either freezedried bloodworms (San Francisco Bay Brand) or flake food (Ocean Star International freshwater aquarium flake food).

\section{Breeding and embryo collection}

Fresh system water was added to 2-3 breeding boxes along with artificial plants. Two female fish and one male fish were added to the breeding box after being fed in the afternoon. The breeding boxes were placed in a water bath at $28^{\circ} \mathrm{C}$. If embryos were present in the morning, they were removed from the breeding box and placed in a petri dish with fresh system water. Embryos that appeared unhealthy or dead were removed, and healthy embryos were kept and sorted for experimentation.

\section{Endocrine-disrupting chemical exposure}

Within an hour of fertilization, wells in a 24-well plate were filled with 5-15 embryos each. The two treatment groups were as follows: vehicle-treated (1.0 of ethanol) and BPA-treated $(17.5 \mu \mathrm{M}$ in ethanol). The embryos were allowed to grow for 24 hours at $28{ }^{\circ} \mathrm{C}$. After 24 hours, any dead embryos were removed from the wells prior to fixation for RNA extraction. If more than five embryos were dead in any single well, the well was not used as a source of embryos.

\section{RNA isolation}

Embryos were removed from wells, and each group was placed into its own sterile 1.5-mL Eppendorf tube. The water was removed, and embryos were homogenized using a manual homogenizer. To each tube, $1.0 \mathrm{~mL}$ of Tri Reagent was added and allowed to sit at room temperature for 5 minutes. After 5 minutes, $200 \mu \mathrm{L}$ of chloroform was added, and the tubes were shaken for 15 seconds, incubated at room temperature for 5 minutes, and centrifuged at $12,000 \mathrm{xg}$ for 15 minutes at $4{ }^{\circ} \mathrm{C}$. Following centrifugation, the aqueous, upper layer was removed and placed into new, sterile 1.5-mL Eppendorf tubes and $500 \mu \mathrm{L}$ of isopropanol was added and allowed to incubate at room temperature for 5 minutes. The tubes were again centrifuged at $12,000 \mathrm{xg}$ for 8 minutes at $4{ }^{\circ} \mathrm{C}$. The isopropanol was removed from each tube without disturbing the RNA pellet. To each tube, $1.0 \mathrm{~mL}$ of $75 \%$ ethanol was added, and tubes were centrifuged at 7,600xg for 5 minutes at $4{ }^{\circ} \mathrm{C}$. The ethanol was removed without disturbing the RNA pellets, and tubes were then allowed to evaporate off any additional ethanol for 5 minutes. RNA pellets were then resuspended in $20 \mu \mathrm{L}$ of DEPC-water at $70{ }^{\circ} \mathrm{C}$ for 15 minutes and then stored in the freezer at $-20{ }^{\circ} \mathrm{C}$. RNA concentrations were determined using a Nanodrop instrument (NanoDrop Lite Spectrophotometer from Thermo Fisher Scientific.) The blank contained $1 \mu \mathrm{L}$ of DEPC-water. Each sample was measured using $1 \mu \mathrm{L}$ of the isolated RNA sample. RNA concentration, 260/280, and 260/230 ratio values were recorded (Table 1).

\begin{tabular}{|c|c|c|}
\hline Sample number & $\mathbf{2 6 0 / 2 8 0}$ & $\mathbf{2 6 0 / 2 3 0}$ \\
\hline 1 & 1.89 & 0.67 \\
\hline 2 & 1.80 & 1.07 \\
\hline 3 & 1.93 & 1.59 \\
\hline 4 & 1.94 & 1.00 \\
\hline 5 & 1.97 & 1.84 \\
\hline 6 & 1.94 & 0.89 \\
\hline 7 & 1.89 & 0.28 \\
\hline 8 & 1.91 & 1.34 \\
\hline 9 & 2.03 & 0.28 \\
\hline
\end{tabular}

Table 1. 260/280 and 230/260 ratios of RNA samples.

\section{cDNA generation}

RNA samples were standardized based on concentration and 260/280 values. RNA samples with 260/280 ratios between 1.80 and 2.00 were used for q-PCR (Table 1). The same amount of RNA (ng) was added to separate PCR tubes, and the volumes were brought up to $5.5 \mu \mathrm{L}$ using DEPC-water. cDNA was generated using reverse transcriptase enzyme from the Thermo Scientific RevertAid Premium First Strand cDNA Synthesis Kit (Fisher product number: ferk1622). To each tube, $0.5 \mu \mathrm{L}$ of oligo $\mathrm{dt}, 2 \mu \mathrm{l}$ of $5 \mathrm{X}$ reaction buffer, $0.5 \mu \mathrm{L}$ of Ribolock RNase inhibitor $(20 \mu \mathrm{g} / \mu \mathrm{L}), 1 \mu \mathrm{L}$ of $10 \mathrm{mM} \mathrm{dNTP}$ mix, and $0.5 \mu \mathrm{L}$ of 
RevertAid M-Mol reverse transcriptase $(200 \mu \mathrm{g} / \mu \mathrm{L})$ was added. The samples were incubated in a thermocycler using the following protocol: 60 minutes at $42{ }^{\circ} \mathrm{C}$ and 3-5 minutes at $70{ }^{\circ} \mathrm{C}$. Samples were then stored at $-20^{\circ} \mathrm{C}$ until they underwent q-PCR.

\section{Quantitative real-time PCR}

qPCR instrument was an Applied Biosystems SteponePlus Real-Time PCR system. In a single RNase-free tube, the following was added for a $10 \mu \mathrm{L}$ master mix reaction: $5 \mu \mathrm{l}$ of $2 \mathrm{X}$ SYBR green master mix (fisher product number: ferk 0373 ), $0.3 \mu \mathrm{L}$ of forward primer, $0.3 \mu \mathrm{L}$ of reverse primer, and cDNA. The primer sets used are listed in Table 2 . All reactions were performed in 96 well plates, in triplicate. Minus RT controls were run in parallel for all primer pairs and RNA samples to monitor DNA contamination. The relative transcription of nanos- 1 in each condition was normalized to $18 S$ and ef1 a levels in the respective conditions using $\Delta \Delta$ CT method. ${ }^{21}$

\begin{tabular}{|c|c|c|}
\hline Gene Name & Forward Primer & Reverse Primer \\
\hline $18 s$ & 5'-TCGCTAGTTGGCATCGT'T'TA-3' & 5'-CGGAGGTTCGAAGACGATCA-3' \\
\hline ef1 a & 5'-CTTCTCAGGCTGACTGTGC-3' & 5'-CCGCTAGCAT'TACCCTCC-3' \\
\hline nanos-1 & 5'-GAGAGCAGCATGGCT'T'T'TC-3' & 5'-T'TCCAAGGCTGAAAGTCCTG-3' \\
\hline \multicolumn{2}{|c|}{ Table 2. Primer sequences used in qPCR experiments. }
\end{tabular}

\section{RESULTS}

In order to ensure that the RNA samples were not degraded, $1 \%$ agarose gels stained with ethidium bromide were used to show proper banding patterns. As shown in Figure 1, the banding pattern of each sample has two bands that are located at the $28 \mathrm{~S}$ and $18 \mathrm{~S}$ positions relative to the RNA ladder.

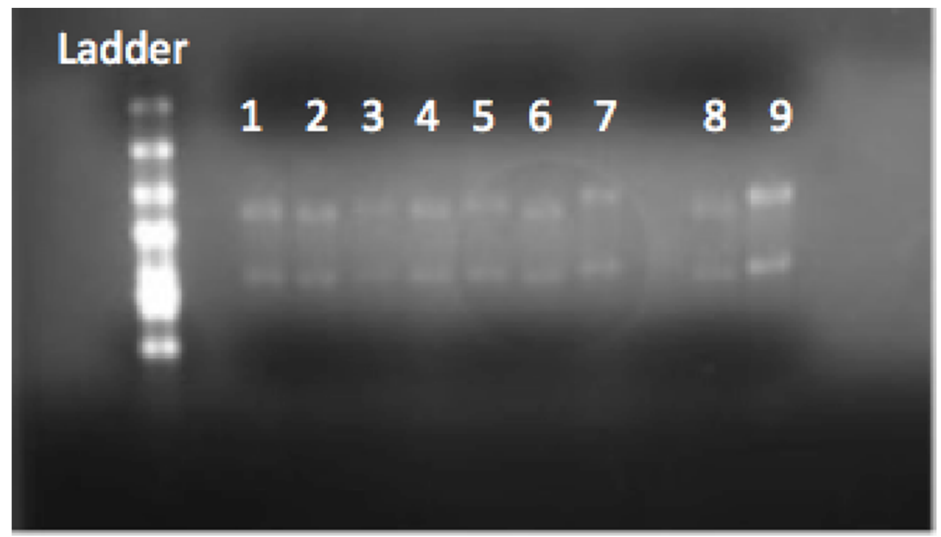

Figure 1. 1\% agarose gel run to check the integrity and quality of RNA samples prior to qPCR. RNA samples on gel represent the samples shown in Table 1.

Once purity, concentration, and stability of the sample were determined to be suitable, several samples were used for q-PCR. Data from the q-PCR was analyzed using $\Delta \Delta C$ T values (Table 3). Analysis of the $\Delta \Delta C T$ values demonstrates that nanos-1 transcription levels do not change following BPA exposure.

\begin{tabular}{|c|c|c|c|c|c|c|c|c|c|c|c|}
\hline \multicolumn{4}{|c|}{ Trial 1} & \multicolumn{4}{|c|}{ Trial 2} & \multicolumn{4}{|c|}{ Trial 3} \\
\hline Treatment & Gene & $\begin{array}{c}\text { Average } \\
\text { CT }\end{array}$ & $\Delta \Delta \mathrm{CT}$ & Treatment & Gene & Average CT & $\Delta \Delta \mathrm{CT}$ & Treatment & Gene & $\begin{array}{c}\text { Average } \\
\text { CT }\end{array}$ & $\Delta \Delta \mathrm{CT}$ \\
\hline \multirow[t]{3}{*}{ Vehicle } & eft $a$ & 10.984 & \multirow[t]{3}{*}{-- } & \multirow[t]{3}{*}{ Vehicle } & eft $a$ & 10.984 & \multirow[t]{3}{*}{-- } & \multirow[t]{3}{*}{ Vehicle } & eft $a$ & 10.686 & \multirow[t]{3}{*}{--} \\
\hline & $18 s$ & 19.109 & & & $18 s$ & 19.109 & & & $18 s$ & 21.470 & \\
\hline & nanos1 & 26.333 & & & nanos1 & 26.333 & & & nanos1 & 23.513 & \\
\hline \multirow{3}{*}{$\begin{array}{c}17.5 \mu \mathrm{M} \\
\mathrm{BPA}\end{array}$} & efla & 11.008 & 0.690 & \multirow[t]{3}{*}{$17.5 \mu \mathrm{M} \mathrm{BPA}$} & eft $a$ & 11.122 & \multirow[t]{3}{*}{0.941} & \multirow{3}{*}{$\begin{array}{c}17.5 \mu \mathrm{M} \\
\mathrm{BPA}\end{array}$} & eft $a$ & 11.021 & 1.329 \\
\hline & $18 s$ & 18.584 & \multirow[t]{2}{*}{0.471} & & \multirow[t]{2}{*}{ nanos1 } & \multirow[t]{2}{*}{26.558} & & & $18 s$ & 21.327 & \multirow[t]{2}{*}{0.954} \\
\hline & nanos1 & 26.891 & & & & & & & nanos1 & 23.437 & \\
\hline
\end{tabular}

Table 3. Raw data from each of the three qPCR experiments. Specifically, $\Delta \Delta C T$ values of nanos-1, in relation to multiple housekeeping genes (ef1 a and $18 s)$, are provided. Each trial represents a biological replicate, and the average CT values represent three reactions for each primer set. 


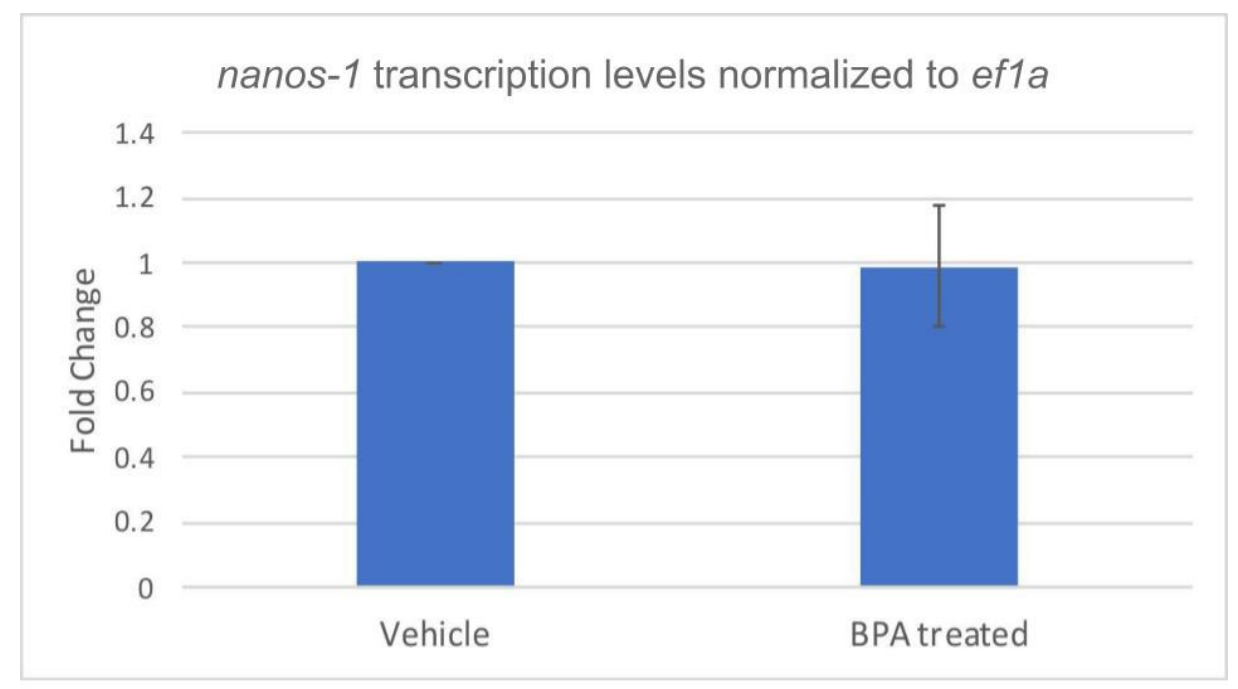

Figure 2. nanos-1 gene transcription levels, normalized to ef1 a levels, from Danio rerio embryos exposed to $17.5 \mu \mathrm{M}$ of BPA, compared to vehicle treatment. The logarithmic scale further illustrates the transcription $\mathrm{n}$ levels of nanos- 1 do not change when exposed to BPA.

As shown in Figure 2, the average $\Delta \Delta C T$ values for the three trials of nanos-1, normalized to ef1 a, are shown in a logarithmic value and only vary from the value of 1 by one-fold. ${ }^{20}$ Similar results were seen when nanos- 1 was normalized to $18 s$. (data not shown). The one-fold difference for the results illustrates that there is no significant change in transcription level of nanos-1 when PGCs are exposed to BPA.

\section{DISCUSSION}

PGCs in zebrafish are ideal cells to study when evaluating the role of endocrine-disrupting molecules in vertebrates. PGCs must travel to and incorporate into the gonad for proper development of the gonad to occur. When PGCs are exposed to BPA, they are unable to migrate correctly. The current study was performed to analyze the role of BPA on the transcription of the germplasm component nanos-1 through q-PCR. It was hypothesized that BPA would alter the transcription levels of nanos-1, thus providing information to help explain the mechanism of BPA-induced abnormal PGC migration. The hypothesis was not supported by the data, as nanos-1 RNA levels remained unchanged following BPA exposures. This result is significant because it allows other aspects of nanos-1 expression, like translation, to become the focus of future studies to understand better the mechanism of action for BPA in disrupting PGC migration. Note that our method, which involves homogenization of the embryos, does not analyze whether BPA affects nanos-1 mRNA levels in a specific, localized region—rather, it analyzes levels throughout the whole embryo. Future studies could analyze whether or not BPA exposure affects nanos-1 levels in specific regions of the embryo.

Overall, this data does not follow the hypothesis of nanos- 1 transcription levels being affected by BPA exposure in zebrafish embryos. The next step is to determine how BPA affects other aspects of PGC migration. This study indicates that other factors in PGC migration must be affected by BPA exposure. Potential targets include nanos-1 at the translational level, genes that control receptors on the germ cell receiving the migration signals, the genes that contribute to the signals being sent to PGCs, or genes that play a role in internal $\mathrm{pH}$ control of PGCs. Once the genes affected by BPA are determined using q-PCR, it would also be appropriate to determine if isoforms of BPA, such as Bisphenol $\mathrm{F}$ or $\mathrm{S}$, also negatively affect PGC migration and if similar genes are affected. It is also possible that BPA affects the expression of these genes at the translational level. Thus it is necessary to complement qPCR with western blot analysis to determine how BPA affects the protein level of these potential targets.

In a 2006 study, the roles of SDF-1 and CXCR4a were analyzed to determine their role in PGC migration. ${ }^{2}$ SDF-1 is a ligand that binds to CXCR4a to ensure proper migration of PGCs. ${ }^{2}$ When there is a loss of function in SDF-1, PGCs are not able to able to migrate correctly. Since our results show that the transcription levels of nanos-1 are not affected by BPA, an important next study would be to determine if BPA affects the ligand-receptor relationship of SDF-1 and CXCR4a. Along with a PGC ligand and receptor possibly being affected by BPA, there is another aspect of PGCs that could be affected and cause abnormal PGC migration. Specifically, the $\mathrm{pH}$ of PGCs was analyzed to understand the role of $\mathrm{pH}$ in PGC migration. ${ }^{22} \mathrm{Ca} 15 \mathrm{~b}$, a gene that contributes to the control of the $\mathrm{pH}$ of PGCs and, subsequently, to their migration patterns, was knocked down with morpholinos. ${ }^{22}$ When there is a loss of function in Ca15b, the internal pH levels become elevated, which causes PGCs to lose their ability to travel the normal migration pathway. Another potential study, therefore, would be to consider the impact of BPA exposure on Ca15b gene expression levels. 


\section{CONCLUSIONS}

Based on recent data from Akbulut, et al., BPA exposure leads to PGC migration defects, as assessed by in situ hybridization. ${ }^{5}$ Their use of in situ hybridization with the vasa gene, a known germ cell marker, allowed them to see the varying locations of PGCs following BPA exposure. ${ }^{5}$ The majority of experimental embryos in the current study did not show clustered PGC staining,

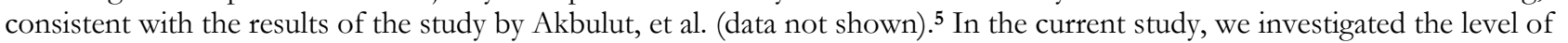
nanos-1 transcription after BPA exposure by measuring RNA levels using qPCR. These experiments were conducted to determine if this gene was affected by BPA exposure at the transcriptional level, as this could provide a novel piece of evidence for a potential mechanism by which EDCs impact PGC migration. nanos- 1 is a highly conserved gene that has been shown to play a crucial role in proper PGC migration during the first $24 \mathrm{hpf} .{ }^{10}$ The lack of a change in nanos-1 transcription levels shown in our study indicates that nanos-1 transcription does not appear to be a target for BPA-induced disruption of PGC migration. Specifically, this is shown by the one-fold or less change in the average $\Delta \triangle \mathrm{CT}$ values for nanos-1 following BPA exposure when normalized to ef1 a and $18 s$ (18s data not shown) (Figure 2). These results suggest that because nanos-1 transcription was not affected by BPA, nanos-1 translation or other genes may be targets of this EDC. Therefore, studying the level of translation for nanos-1, as well as the overall expression of genes other than nanos-1 may help to explain the impact of BPA exposure on PGC migration.

It is well-established in the current scientific literature that BPA has adverse effects on zebrafish development. ${ }^{5,15}$ It is also known that BPA can have adverse effects on human health. ${ }^{12}$ Little is known about the mechanism by which BPA disrupts endocrine function, and this research aims to shed light on that mechanism. Zebrafish are particularly useful as a model organism for analyzing the mechanism of action of EDCs in vertebrates. Therefore, this research can shed light on the mechanism by which BPA affects both zebrafish and humans. ${ }^{23}$ Knowledge of BPA's mechanism of action is relevant to both human health and, from an ecological perspective to the health of aquatic organisms.

\section{ACKNOWLEDGMENTS}

Authors thank Jennifer Gantress, Lab Manager, for purchasing supplies and St. John Fisher College, for providing budgetary support.

\section{REFERENCES}

1. Paksa, A. and Raz, E. (2015) Zebrafish germ cells: motility and guided migration, Curr Opin Cell Biol, 36, 80-85. bttps:// doi.org/10.1016/j.ceb.2015.07.007

2. Raz, E. and Reichman-Fried, M. (2006) Attraction rules: germ cell migration in zebrafish, Curr Opin Genet Dev, 16(4), 355-359. https:// doi.org/10.1016/j.gde.2006.06.007

3. Fujimoto, T., Miyayama, Y., and Fuyuta, M. (1977) The origin, migration and fine morphology of human primordial germ cells, Anat Rec, 188(3), 315-329. bttps:/ / doi.org/10.1002/ ar.1091880305

4. Wear, H.M., McPike, M., and Watanabe, K. (2016) From primordial germ cells to primordial follicles: a review and visual representation of early ovarian development in mice, J Ovarian Res, 9:36. https:// doi.org/10.1186/s13048-016-0246-7

5. Akbulut, C., Kizil, A., and Yon, N.D. (2013) Effects of low doses of bisphenol A on primordial germ cells in zebrafish (Danio rerio) embryos and larvae, Kafkas Universitesi Veteriner Fakilltesi Dergisi, 19(4), 647-653. https:/ / doi.org/10.9775/ kvfd.2013.8600

6. Yön, N. and Yüce, R. (2013) Effect of deltamethrin on primordial germ cells in zebrafish (Danio rerio) Embryos, Fresenius Environmental Bulletin, 22(11), 3217-3222.

7. Boldajipour, B., Mahabaleshwar, H., Kardash, E., Reichman-Fried, M., Blaser, H., Minina, S., Wilson, D., Xu, Q., and Raz, E. (2008) Control of chemokine guided cell migration by ligand sequestration, Cell,132(3), 463-473.

https:// doi.org/10.1016/j.cell.2007.12.034.

8. Weidinger, G., Stebler, J., Slanchev, K., Dumstrei, K., Wise, C., Lovell-Badge, R., Thisse, C., Thisse, B., and Raz, E. (2003) Dead end, a novel vertebrate germ plasm component, is required for zebrafish primordial germ cell migration and survival, Curr Biol, 13(16), 1429-1434.

9. Liu, W. and Collodi, P. (2010) Zebrafish dead end possesses ATPase activity that is required for primordial germ cell development, FASEB J, 24(8), 2641-2650. https:// doi.org/10.1096/fj.09-148403

10. Koprunner, M., Thisse, C., and Raz, E. (2001) A zebrafish nanos-related gene is essential for the development of primordial germ cells, Genes Dev, 15(21), 2877-2885.

11. Willey, J.B. and Krone, P.H. (2001) Effects of endosulfan and nonyphenol on the primordial germ cell population in pre-larval zebrafish embryos, Aquat Toxicol, 54,(1-2), 113-123. bttps:/ mmnv.ncbi.nlm.nih.gov/pubmed/11451430

12. Rezg, R., El-Fazaa, S., Gharbi, N., and Mornagui, B. (2014) Bisphenol A and human chronic diseases: current evidences, possible mechanisms, and future perspectives, Environ Int, 64, 83-90. bttps:// doi.org/10.1016/j.envint.2013.12.007

13. Tsai, W. T. (2006) Human health risk on environmental exposure to bisphenol-A: a review, J Environ Sci Health C Environ Carcinog Ecotoxicol Rev, 24(2), 225-255. bttps: / / doi.org/10.1080/10590500600936482 
14. Rhee, J. S., Kim, B. M., Lee, C. J., Yoon, Y. D., Lee, Y. M., and Lee J. S. (2011) Bisphenol A modulates expression of sex differentiation genes in the self-fertilizing fish, Kryptolebias marmoratus, Aquat Toxicol, 104(3-4), 218-229.

bttps://doi.org/10.1016/j.aquatox.2011.04.020

15. Chen, J., Saili, K.S., Liu, Y., Li, L., Zhao, Y., Bai, C., Tanguay, R.L., Dong, Q., and Huang, C. (2017) Developmental bisphenol A exposure impairs sperm function and reproduction in zebrafish. Chemosphere 169, 262-270.

https:/ / doi.org/ 10.1016/j.chemosphere.2016.11.089

16. Corrales, J., Kristofco, L.A., Steele, W.B., Yates, B.S., Breed, C.S., Williams, E.S., and Brooks, B.W. (2015) Global assessment of bisphenol A in the environment: review and analysis of its occurrence and bioaccumulation. Dose-Response 13, 1-29.

https:// doi.org/10.1177/1559325815598308

17. Fukazawa, H., Watanabe, M., Shiraishi, F., Shiraishi, H., Shiozawa, T., Matsushita, H., and Terao, Y. (2002) Formation of chlorinated derivatives of bisphenol A in waste paper recycling plants and their estrogenic activities. J Health Sciences 48, $242-249$. bttps://doi.org/10.1248/jhs.48.242

18. Urase, T. and Miyashita, K. (2003) Factors affecting the concentration of bisphenol A in leachates from solid waste disposal sites and its fate in treatment processes. J Mater Cycles Waste Manag, 77-82. http:/ / dx.doi.org/10.1007/s101630300012

19. Eladak, S., Grisin, T., Moison, D., Guerquin, M.J., N’Tumba-Byn, T., Pozzi-Gaudin, S., Benachi, A., Livera, G., RouillerFabre, V., and Habert, R. (2015) A new chapter in the bisphenol A story: bisphenol S and bisphenol F are not safe alternatives to this compound. Fertil Steril 103, 11-22. https:// doi.org/10.1016/j.fertnstert.2014.11.005

20. McCurley, A. T. and Callard, G. V. (2008) Characterization of housekeeping genes in zebrafish: male-female differences and effects of tissue types, developmental stage and chemical treatment, BMC Mol Biol, 9, 102-102. https://doi.org/10.1186/1471-21999-102

21. Livak, K. J. and Schmittgen, T. D. (2001) Analysis of relative gene expression data using real-time quantitative PCR and the 2- $\Delta \Delta$ CT Method. Methods, 25, 402-408. https:/ / doi.org/ 10.1006/ meth.2001.1262

22. Tarbashevich, K., Reichman-Fried, M., and Raz E. (2015) Chemokine-dependent pH elevation at the cell front sustains polarity in directionally migrating zebrafish germ cells, Curr Biol, 25(8), 1096-1103. DOI: 10.1016/j.cub.2015.02.071

23. Segner, H. (2009) Zebrafish (Danio rerio) as a model organism for investigating endocrine disruption. Comp Biochem Physiol C

Toxicol Pharmacol, 149(2), 187-195. https:// doi.org/10.1016/j.cbpc.2008.10.099

\section{ABOUT STUDENT AUTHORS}

Bridget Babich graduated with a BS in Biology from St. John Fisher College in 2015 and graduated from Physician's Assistant (PA) school in New Hampshire at the Manchester Campus of the Massachusetts College of Pharmacy and Health Sciences in 2018. She will be working in Surgical Urology at the Brigham and Women's Hospital in Boston. George Roba graduated with a BS in Biology, a BA in Psychology, and a minor in Spanish for the Health Care Professional from St. John Fisher College in 2018. He successfully completed paramedic training in May 2019 and is currently pursuing a career in healthcare. Siti Sarah Safura graduated with a BS in Biology with minors in Chemistry and Women \& Gender Studies from St. John Fisher College in 2017 and an MS in Medical Humanities from the University of Rochester in 2018. She is currently pursuing a career in medicine.

\section{PRESS SUMMARY}

This project was done to determine if nanos-1, a gene critically important in zebrafish primordial germ cell migration, is affected by BPA exposure. BPA and itanalogses are commercially used plasticizers that are known to leach out of consumer products.

Zebrafish are a commonly used model organism and are excellent models to analyze the effects of EDCs on PGC migration, a process that also occurs in humans. Proper PGC migration is necessary in vertebrates for proper gonad formation. BPA is known to disrupt PGC migration in zebrafish; however, the mechanism(s) by which this occurs is unknown. This project found that BPA does not affect nanos-1 RNA levels during PGC migration, and therefore helps rule out nanos-1 transcription as a potential target for BPA in causing abnormal PGC migration in zebrafish. 


\title{
Investigation of Atmospheric Aerosol Size Distributions from Ground- Based Solar Spectrometer Measurements Synthesized with Satellite Data
}

\author{
Dane T. Kubr* \& Adam T. Whitten \\ Physics Department, College of Saint Benedict/Saint John's University, Collegeville, $M N$ \\ bttps://doi.org/10.33697/ajur.2019.013 \\ Student:dkubr001@csbsju.edu* \\ Mentor:awbitten@csbsju.edu
}

\begin{abstract}
Data collected by a ground-based solar spectrometer at Collegeville, MN, was used to generate Aerosol Optical Depths (AODs) throughout the 2017 calendar year. The AOD data was then visualized at 13 selected wavelengths throughout the year and analyzed in comparison to satellite imagery, upper air charts and backwards trajectories of air masses moving towards Central Minnesota in order to determine key dates of interest that correspond to times before (20170615), during (20170729), and at the conclusion of (20170914) forest fires that burned in British Columbia (BC) during the summer of 2017. The data from these specific days were analyzed further by inputting the maximum and minimum AODs for each day into a Parameter Based Particle Swarm Optimization (PBPSO) algorithm in order to generate bimodal lognormal particle size distributions. The bimodal distributions were chosen because they carry more information about the aerosol loads across the entire spectrum of particle radii. The resulting distributions show an increase in number density and decrease in median radius in the Aitken mode during the BC forest fires and a relatively constant (within uncertainty) number density of accumulation mode particles at daily maximum AODs. Comparing the resulting bimodal lognormal distribution for daily minimum AODs (where evaporation and other diurnal effects are at a minimum) shows an increased number density of Aitken mode particles by two orders of magnitude from pre- to postforest fires. This measured increase in the number density of smaller radii particles due to forest fires illustrates the PBPSO's capability of distinguishing variations in atmospheric aerosol particle number size distributions in the Aitken mode based on data collected by the Kipp-Zonen PGS-100 solar spectrometer.
\end{abstract}

\section{KEYWORDS}

Atmospheric Aerosol; Particle Swarm Optimization; Aerosol Optical Depth; Solar Spectrometer; Size Distributions; Forest Fire; Satellite Imagery; Upper Air Charts; Backward Trajectory

\section{INTRODUCTION}

Atmospheric aerosols are of interest to members of the scientific community and the general public alike because they affect environmental conditions and public health. ${ }^{1-3}$ The environmental effects of aerosols depend on the particles' radii, ranging from $.001-100 \mu \mathrm{m},{ }^{2}$ chemical composition, and optical properties. ${ }^{3}$ Atmospheric aerosols contribute to regional climate change by absorbing and scattering radiation and acting as cloud condensation nuclei (CCN)., 5 Natural aerosols tend to be larger particles and are formed of plant matter, ash, sea salt, dust, and other aqueous chemicals. Anthropogenic aerosols, on the other hand, tend to be sub-micrometer particles formed by combustion emissions and in some cases are known toxins. ${ }^{6}$ These smaller anthropogenic aerosols are capable of traveling farther down the respiratory tract posing a major threat to public health. ${ }^{6}$ All atmospheric aerosols impact air quality ${ }^{3}$, visibility ${ }^{4}$ and climate change. ${ }^{7}$

Ground-based solar spectrometers are commonly used to measure solar irradiance values that relate to aerosol optical depth (AOD). In order to calculate AODs from irradiances measured by the PGS-100 spectrometer, it is necessary to calculate the irradiance across the entire spectrum. The irradiance measured at Earth's surface can be related to the irradiance at the top of Earth's atmosphere by taking into account absorption, Rayleigh scattering, and Mie scattering. Equation 1, outlined in London et al. 1985, yields irradiance as a function of wavelength: ${ }^{8}$

$$
L_{\lambda}=\frac{L_{\lambda_{0}}}{D^{2}}\left(e^{-\alpha \mathrm{X} \mu-m \beta \frac{P}{P_{0}}-\delta m^{\prime}}\right)
$$

Equation 1.

where $L_{\lambda_{0}}$ is the irradiance at the top of Earth's atmosphere at a distance of $1 \mathrm{AU},{ }^{9} L_{\lambda}$ is the irradiance measured by the PGS-100 spectrometer, $D$ is the distance from the Earth to the Sun in astronomical units (AU), $\alpha$ is the absorption coefficient of the 
absorption species in $\mathrm{cm}^{-1}, \mathrm{X}$ is the total thickness of absorber in a vertical column at STP (cm), and $\mu$ is the ratio of actual and vertical paths of the light through the absorbing layer, $m$ is the optical path length allowing for refraction (atm), $\beta$ is the atmospheric Rayleigh scattering coefficient (atm), $P$ is the pressure at the point of measurement ( $\mathrm{mb}$ ), $P_{0}$ is the mean sea level pressure (mb), $\delta$ is aerosol optical depth (dimensionless) and $m^{\prime}$ is $\sec \theta$ where $\theta$ is the solar zenith angle. ${ }^{8}$

Because this investigation focuses on 13 wavelengths avoiding absorption regions, the first term in the exponential in Equation 1 becomes negligible, and inversion into Equation 2 enables the calculation of aerosol optical depths based on measured irradiances.

$$
\delta=\frac{\ln \left(\frac{L_{\lambda} D^{2}}{L_{\lambda_{0}}}\right)-m \beta \frac{P}{P_{0}}}{m^{\prime}}
$$

Equation 2.

Instead of using Komhyr's expression for Rayleigh optical depth $\left(m \beta \frac{P}{P_{0}}\right)$, which assumes an atmospheric $\mathrm{CO}_{2}$ concentration of $300 \mathrm{ppm}$, this research uses the recommendation of Bodhaine et al. 1999. ${ }^{10}$ Bodhaine's method takes $\mathrm{CO}_{2}$ concentration into account by including its contribution to the scattering cross section of air (including the molecular mass, the index of refraction, density and the depolarization term or King factor). This research uses $\mathrm{CO}_{2}$ concentration measured at the Mauna Loa Observatory in Hawaii, resulting in Equation 3:

$$
\delta=\frac{\ln \left(\frac{L_{\lambda_{0}}}{L_{\lambda} D^{2}}\right)}{m^{\prime}}-\sigma \frac{P A}{m_{a} g}
$$

Equation 3.

where $L_{\lambda_{0}}, L_{\lambda}, D, P$ and $m^{\prime}$ all represent the same values as above, $\sigma$ is the scattering cross section per molecule that accounts for increased partial pressure of increasing atmospheric $\mathrm{CO}_{2}$ levels, $A$ is Avogadro's number, $m_{a}$ is the mean molecular mass of air $(\mathrm{kg})$ and $g$ is the acceleration of gravity at the measurement site. ${ }^{10}$

A Parameter Based Particle Swarm Optimization algorithm described by DuPaul et al. 2017 can be applied to these inferred AODs in order to retrieve particle size distributions. ${ }^{11}$ This yields valuable information about the atmospheric aerosols which are highly heterogeneous in time and space. The PBPSO provides a reasonable, but computationally expensive means to find particle number size distribution fit parameters based on input AOD data. Bimodal distributions are of the form:

$$
n_{B}(r)=\frac{d N(r)}{d \ln (r)}=\frac{N_{0}}{\sqrt{2 \pi}} \frac{1}{\beta_{0}} e^{-.5\left[\frac{\ln \left(r / \alpha_{0}\right)}{\beta_{0}}\right]^{2}}+\frac{N_{1}}{\sqrt{2 \pi}} \frac{1}{\beta_{1}} e^{-.5\left[\frac{\ln \left(r / \alpha_{1}\right)}{\beta_{1}}\right]^{2}}
$$

Equation 4.

where $r$ is the particle radius in $\mu \mathrm{m}, N_{0}$ is the number density of particles in the Aitken mode in $\mathrm{cm}^{-3}$ and $\alpha_{0}$ and $\beta_{0}$ are the median radius in $\mu \mathrm{m}$ and the width of the Aitken mode, respectively; while $N_{1}, \alpha_{1}$, and $\beta_{1}$ are the corresponding parameters for the accumulation mode (note that $\beta=\ln (\mathrm{s})$ where $\mathrm{s}$ is the geometric standard deviation). ${ }^{11}$

The PBPSO algorithm generates 200 possible solutions and then calculates AODs using standard Mie theory for each of the 13 wavelengths for measured AODs. Particle Swarm Optimization algorithms are iterative algorithms that move a set of randomly generated solutions towards the solution that gives the best agreement with measured data. The $\overline{\chi^{2}}$ for the observed and calculated AODs is used to identify local minima for a single solution and a global minimum for the set of 200 solutions. All solutions are moved towards these minima with each iteration until the distribution parameters of the global minimum have changed by less than $.001 \%$ for 100 consecutive iterations. This process is repeated ten times so that a mean and standard deviation of the mean can be calculated for $N_{0}, \alpha_{0}, \beta_{0}, N_{1}, \alpha_{1}$, and $\beta_{1}$.

The comprehensive satellite images and atmospheric measurements from the National Aeronautics and Space Administration (NASA), National Oceanic and Atmospheric Administration (NOAA) and National Weather Service (NWS) provide an approach to verify the findings of ground-based solar spectrometers. Satellite data containing visual spectrum images, aerosol optical depth gradients, upper air charts, backward trajectory maps, and forest fire characteristics prove invaluable in determining where aerosol loads travel, how air moves at different pressures and altitudes in the atmosphere and what events cause deviations in aerosol optical depths around the world. ${ }^{12-14}$

Conducting atmospheric aerosol research in Central Minnesota provides an interesting perspective as the aerosol load should be substantially less than in metropolitan areas experiencing heavy anthropogenic aerosol emissions. ${ }^{5,15-17}$ By cross-referencing data 
produced by Saint John's University's solar spectrometer with various satellite images, backward air mass trajectories, and upper air charts in a similar manner as Niemi et al. $2015,{ }^{15}$ it became possible to examine changes in particle size distributions during events that alter the composition and amount of aerosols present.

One such event occurred in the summer of 2017 when British Columbia (BC) exhibited ideal conditions to spark massive forest fires. ${ }^{18}$ These fires burned over 1.2 million hectares (over 4,600 square miles) and emitted an estimated 200 million tons of carbon dioxide equivalent into the atmosphere. ${ }^{18,19}$ The effects of these aerosols being introduced into the atmosphere have a short lifetime, but the immediate atmospheric effects can be seen by investigating the AOD and particle size distributions before, during, and at the end of the BC forest fires of 2017.

\section{METHODS AND PROCEDURES}

This research investigates the data from a ground-based solar spectrometer compared to NASA's Earth Observatory AOD data, NOAA Upper Air Charts, and satellite imagery from the GOES-13 satellite. ${ }^{20}$ The following steps outline how the data collected at Saint John's University was used in conjunction with satellite imagery and other resources to determine how particle size distributions changed throughout an episode related to the BC fires. The analysis procedure followed these steps:

Step 1: Analysis of the spread of the major BC forest fires of 2017 revealed that the original fires commenced after severe weather systems passed through on July 6-8, 2017 (day-of-year 187-189) and spread rapidly, culminating in the most massive fires connecting in Mid-August and the State of Emergency ending on September 15 (day 258). ${ }^{18}$

Step 2: Before analysis of the raw data from the solar spectrometer could begin, local atmospheric pressure data were obtained from the NOAA Radiosonde database, and global atmospheric $\mathrm{CO}_{2}$ data was collected from the Mauna Loa Observatory database with daily values interpolated from the given weekly averages. ${ }^{21,22}$ The $\mathrm{CO}_{2}$ and local pressure levels influence the calculated AOD and therefore affect the particle size distributions and the other products of this research.

Step 3: The data collected by the Kipp-Zonen PGS-100 Solar Spectrometer was converted from raw count data from the spectrometer into irradiance values by applying the manufacturer's calibration constants. The data were screened to eliminate measurements with significant cloud cover, judged by raw counts below 20,000 at $778 \mathrm{~nm}$. This wavelength was chosen as it is just past the large $\mathrm{O}_{2}$ absorption region and is not subject to strong Rayleigh scattering.

Step 4: The aforementioned atmospheric $\mathrm{CO}_{2}$ measurements from Step 2 were included in Equation 3 in order to calculate AOD across all wavelengths taking into account the new Rayleigh scattering term with adjustments for increasing $\mathrm{CO}_{2}$ levels. Details of this calculation are provided in Bodhaine et al. 1999.10

Step 4: Each day's AOD at thirteen key wavelengths was extracted: the standard World Meteorological Organization's (WMO) wavelengths: 412, 500, 610, 675, and $778 \mathrm{~nm}$; WMO wavelengths shifted out of absorption regions: 463,870 and $1020 \mathrm{~nm}$; and five additional wavelengths: 441, 479, 520, 556, $750 \mathrm{~nm} .{ }^{23}$ These specific wavelengths enable the visualization of aerosol optical depth without absorption from common gaseous atmospheric components.

Step 5: In order to better understand the AOD trends over Central Minnesota, Figure 1 was constructed using all of the AOD data from 2017. This gave valuable insights as to which date ranges would provide viable information.

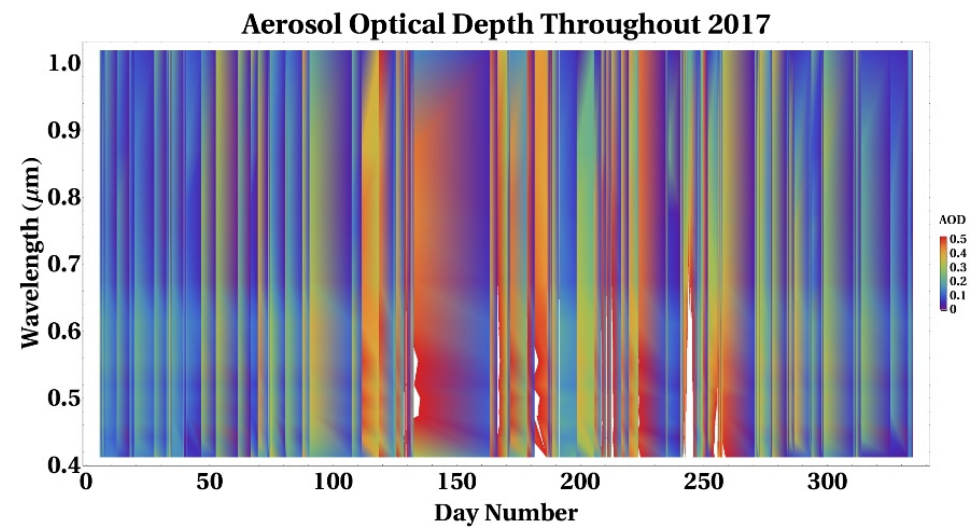

Figure 1. Surface visualization of the entire year (2017) of AOD data. This enabled the selection of viable date ranges around the time of the BC forest fires. The gaps in the data seen clearly between days 130-160 are due to significant cloud cover. 
Step 6: Comparing the AOD data in Figure 1 to the timeline of the BC forest fire revealed three preliminary dates of interest that correspond to times before, during, and after the wildfires. These dates: 15 June (166), 29 July (210) and 14 September (257) contain sufficient AOD data (significant cloud cover was not an issue).

Step 7: Figure 2 shows higher time-resolution spectra on the three specific dates of interest leading to a hypothesis that interesting particle size distribution data would be present during the times of the daily minimum and maximum AOD on each of these days.

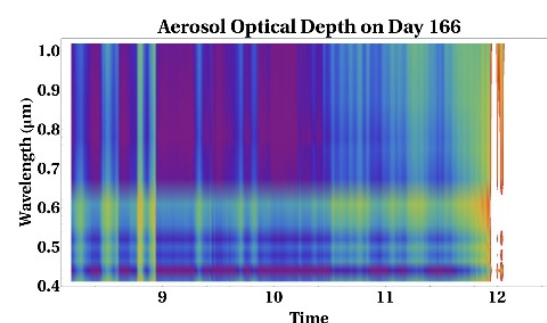

Figure 2.1

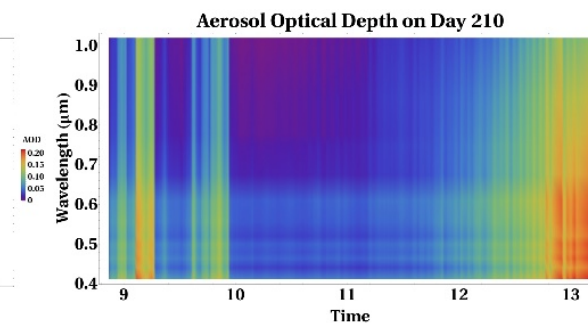

Figure 2.2

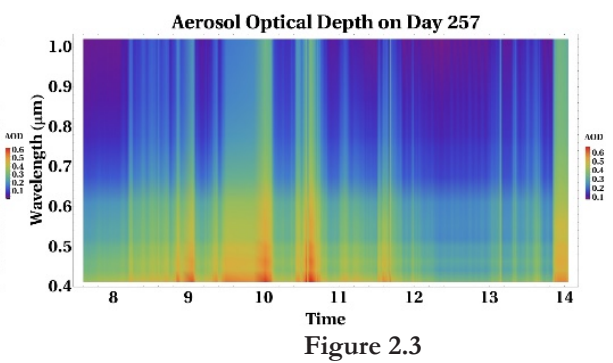

Figure 2.3

Figure 2. Surface plots of calculated AOD values. Figure 2.1 illustrates day 166 (15 June 2017), Figure 2.2 illustrates day 210 (29 July 2017 ), and Figure 2.3 illustrates day 257 (14 September 2017) in greater detail. The solar spectrometer re-aligning itself with the center of the sun's maximum intensity causes the vertical lines seen in these plots and the white sections, as seen on the far right of Figure 2.1 illustrate what occurs when the spectrometer excludes data due to cloud cover.

Step 8: The six selected times (maxima and minima AOD data on the three days) were then extracted from Figure 2 and illustrated in Figure 3 to show the variations in AOD across the spectrum of wavelengths at each time.

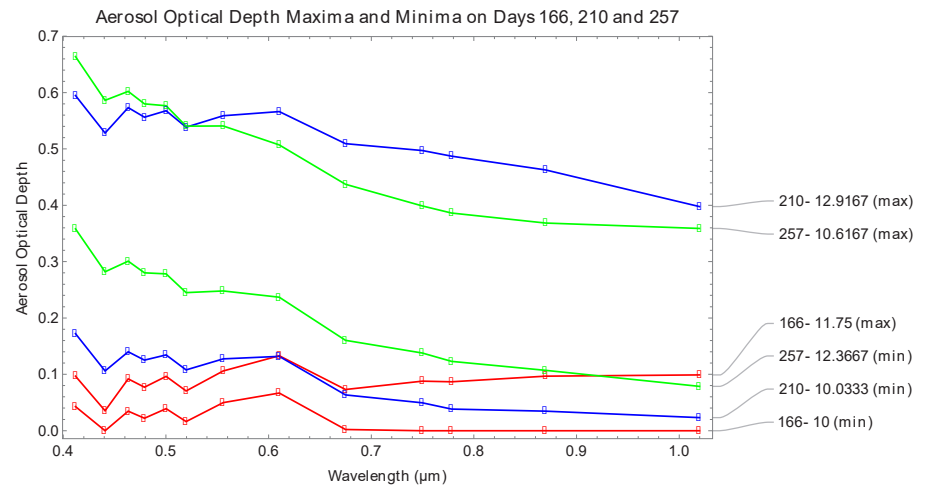

Figure 3. Graph of maxima (squares) and minima (circles) AOD on days 166, 210 and 257 depicts a clear increase in AOD at all wavelengths from June to July and September.

Step 9: To better understand the variations in AOD data throughout each day, the plots in Figure 2 were cross-referenced with satellite imagery in Figure 4 from NASA's GOES 13 geostationary satellite to determine if cloud cover or other sources of atmospheric aerosols likely caused the fluctuation in AOD data.

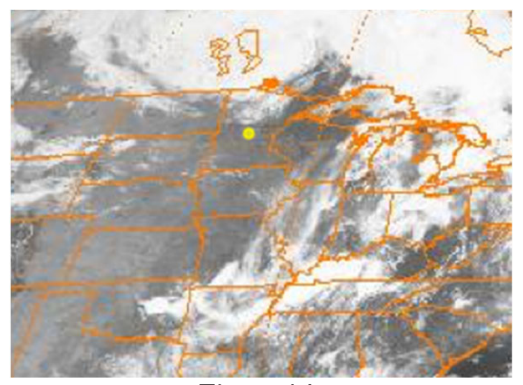

Figure 4.1

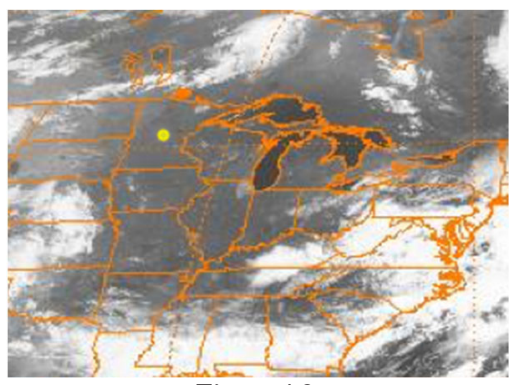

Figure 4.2

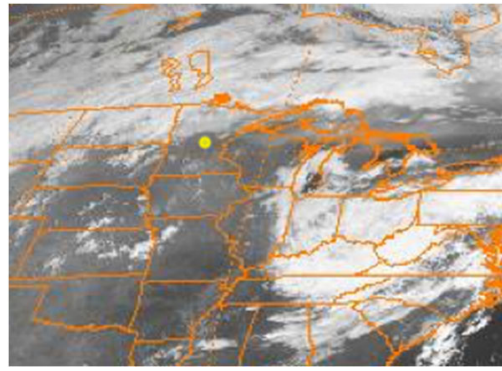

Figure 4.3

Figure 4. Satellite images from GOES 13 satellite showing cloud cover on 15 June 2017 in Figure 4.1, 29 July 2017 in Figure 4.2 and 14 September 2017 in Figure 4.3 each captured at 1445 UTC (9:45 a.m. local time in Minnesota). ${ }^{13}$ The yellow dot indicates the location of the solar spectrometer where data was collected. 
Step 10: Once the times of interest (maximum and minimum AOD) on days 166, 210, and 257 had been selected, the PBPSO algorithm was used to find the fit parameters of the bimodal lognormal particle size distribution shown in Equation $4 .^{11}$ The variables in Equation 4 correspond to different aspects of the bimodal distribution where $r$ is the particle radius in $\mu \mathrm{m}, N_{0}$ is the number density of particles in the Aitken mode in $\mathrm{cm}^{-3}$, and $\alpha_{0}$ and $\beta_{0}$ are the median radius in $\mu \mathrm{m}$ and the width of the Aitken mode, respectively; while $N_{1}, \alpha_{1}$, and $\beta_{1}$ are the corresponding parameters for the accumulation mode.

\section{RESULTS AND DISCUSSION}

Based on the evidence shown in Figure 4 that heavy cloud was not present over the site on the three days of interest, Upper Air Charts provided by National Oceanic and Atmospheric Administration (NOAA) and the National Weather Service (NWS) are used to illustrate the direction of air traveling from the $\mathrm{BC}$ region to the upper Midwest and Central Minnesota more specifically. ${ }^{24}$ Figure 5 shows the backward trajectories from NOAA Hybrid Single-Particle Lagrangian Integrated Trajectory (HYSPLIT) models, ${ }^{13}$ illustrating the plausibility that the particles in the atmosphere above Central Minnesota traveled from the Pacific Northwest or Southern BC, corresponding to the region of the major forest fires in this time period. An exception was found on day 257 when the HYSPLIT backward trajectories showed air masses moving towards Central Minnesota from the Pacific Northwest, but the NOAA upper air charts showed trajectories coming from Central California. This could elicit the irregular trend in AOD data on day 257, but does not change the results of the size distributions.

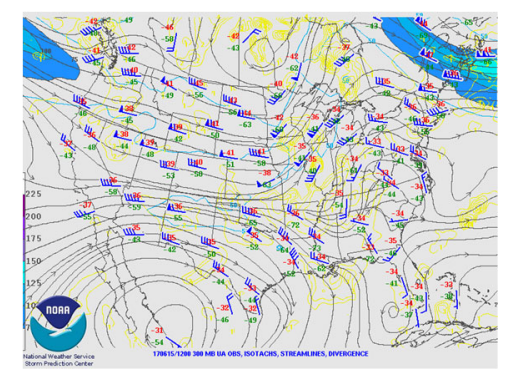

Figure 5.1. Day 166 NOAA HYSPLIT MODEL Backward trajectories ending at 1900 UTC 15 Jun 17

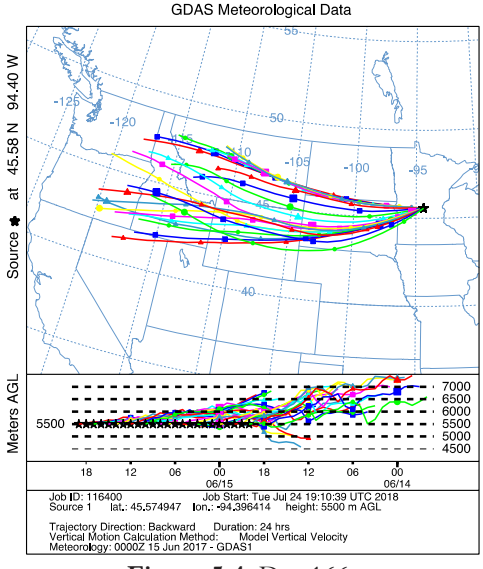

Figure 5.4. Day 166

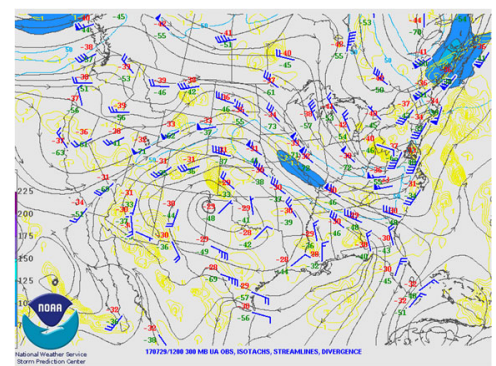

Figure 5.2. Day 210

NOAA HYSPLIT MODEL GDAS ending at 1900 UTC 29 Jul 17 Backward trajectories ending at 1900 GDAS Meteorological Data
GDo

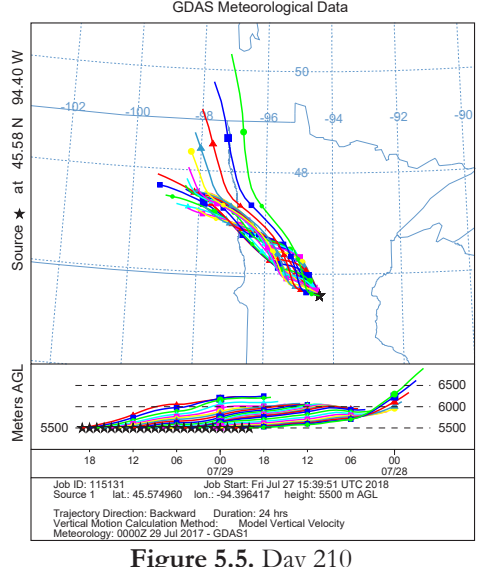

Figure 5.5. Day 210

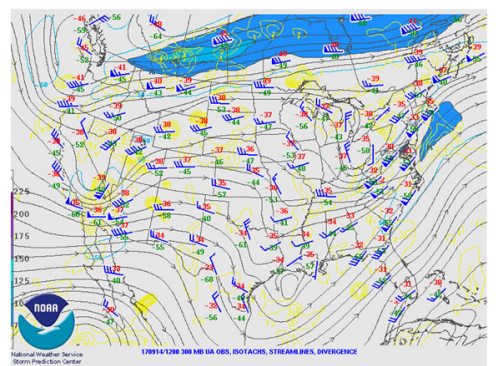

Figure 5.3. Day 257 NOAA HYSPLIT MODEL GDAS Meteorological Data

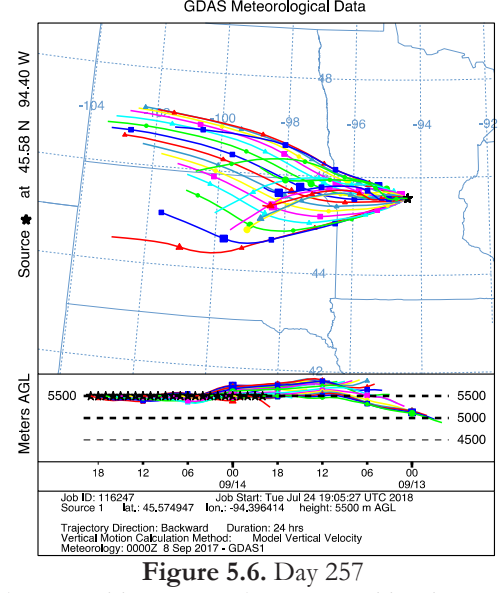

Figure 5. Figures 5.1-5.3 NOAA Storm Prediction Center Upper Air charts depicting the air patterns at $300 \mathrm{mb}$ or roughly $5500 \mathrm{~m}$ above ground level. Figure 5.4-5.6 NOAA HYSPLIT backward trajectories of air masses $5500 \mathrm{~m}$ above ground level (AGL) ending at the Saint John's University observatory where the solar spectrometer is located. ${ }^{10}$

With the understanding that on days 166, 210, and 257 the atmospheric aerosols above Central Minnesota likely came from near BC, the particle size distributions in Figures 6-8 carry information regarding how the combustion products suspended in the atmosphere from the forest fire affected particle size distributions. Chemically processed particles from combustion contribute to the Aitken mode portion of the bimodal distribution while mechanically generated particles contribute to the accumulation mode. As an air mass ages, it is expected that collisions will shift all particles toward larger radii.

On the specific days of interest, the PBPSO algorithm was run to generate bimodal distributions while allowing all fit parameters to vary. Based on information in Hodzic et al. 2007, forest fires primarily (80-90\% by mass) emit aerosols with radii $<1.0 \mu \mathrm{m},{ }^{25}$ the bimodal distributions should illustrate how the aerosol loads changed with respect to the smaller particles with radii in the Aitken mode $(.02-.10 \mu \mathrm{m})$, but aerosols in the accumulation mode $(.10-1.0 \mu \mathrm{m})$ may form as the air mass ages during transport. ${ }^{3}$ Analysis 
of the bimodal distribution parameters accurately quantifies the broad spectrum variations in atmospheric aerosol loads as evidenced by their low $\overline{\chi^{2}}$ averages. These distributions, illustrated in Figures 6-8, highlight the transformation of both the Aitken and accumulation modes from day 166 to 210 to 257 at both the daily minimum and maximum AOD.

Diurnal Comparisons

On each of the selected days, different trends arise in the fit parameters between the minima and maxima AOD. The resulting parameters show that on each of the selected days, the number density of accumulation mode particles increases from the minimum to maximum AOD, while the median radius and width of distribution in the accumulation mode remain constant within uncertainty. Additionally, on the two days when BC forest fire aerosols are possibly present, the number density of Aitken mode particles is also constant within uncertainty. This suggests that throughout any given day, the number of larger particles and AOD are positively correlated, but when smoke and ash particles are likely present (Tables $\mathbf{2}$ and $\mathbf{3}$ ), the number of smaller radii particles remains stable with increasing AOD.

\section{Daily Minima Comparisons}

To better understand the results of the distributions in regards to potential smoke in the atmosphere, each distribution fit parameter was evaluated. When looking at the Aitken mode parameters of the daily minima AODs, the $\mathrm{N}_{0}$ increases by roughly an order of magnitude from day 166 to 210 and again from 210 to 257, $\alpha_{0}$ decreases by about 20\% from each day to the next, $\beta_{0}$ is constant within uncertainty on days 166 and 210 but nearly doubles on day 257. Looking at the accumulation mode parameters, $\mathrm{N}_{1}$ remains constant within uncertainty on days 166 and 210 and increases by 100 on day 257, $\alpha_{1}$ increases from day 166 to 210 and remains constant within uncertainty on days 210 and 257, and $\beta_{1}$ remains constant within uncertainty between days 166 and 210 and also between days 210 and 257. Analyzing how these parameters evolve over time illustrates how each mode of particles changes.

The steady increase in $\mathrm{N}_{0}$ indicates many more particles with radii between $.01-.10 \mu \mathrm{m}$ present on days 210 and 257 compared to 166. Based on the steady decrease in $\alpha_{0}$, the median radius of these small particles decreases, which suggests an influx of small radii aerosols, consistent with combustion aerosols from forest fire emissions. The steady $\beta_{0}$ values depict a relatively constant spread in particle radii throughout the range of dates.

The PBPSO algorithm has difficulties illustrating the trends of larger particles in bimodal distributions due to the relatively flat Mie extinction profiles at larger radii. ${ }^{11}$ The accumulation mode results show that as time progressed, the median radius, $\alpha_{1}$, increased from day 166 to day 210 and then the number of particles, $N_{1}$, increased from day 210 to day 257 while the width of the distribution, $\beta_{1}$, remained constant within uncertainty across all of the days.

\section{Daily Maxima Comparisons}

The size distributions from the daily maxima AOD showed a similar trend as the daily minima in the fit parameters $\mathrm{N}_{0}$ and $\alpha_{0}$ illustrating an increase in particles in the Aitken mode with smaller median radii, but the $\beta_{0}$ values indicate a wider distribution of radii from day 166 to day 210 and then a narrower distribution from day 210 to day 257. This suggests that the increase in Aitken mode aerosols was due to an influx of smaller radius particles. Another interesting result arises in the accumulation mode of the daily maxima AOD size distributions where $\mathrm{N}_{1}$ and $\alpha_{1}$ values remain constant within uncertainty across all three days, and $\beta_{1}$ is seen to increase from day 166 to day 210 and then remain constant within uncertainty. This result suggests that while the number and median radius of particles remains constant, the variation in particle radius in the accumulation mode may increase when smoke from forest fires is present due to the rapidly changing size distributions caused by differences in transport height. 
Day 166 Minimum AOD at 10:00

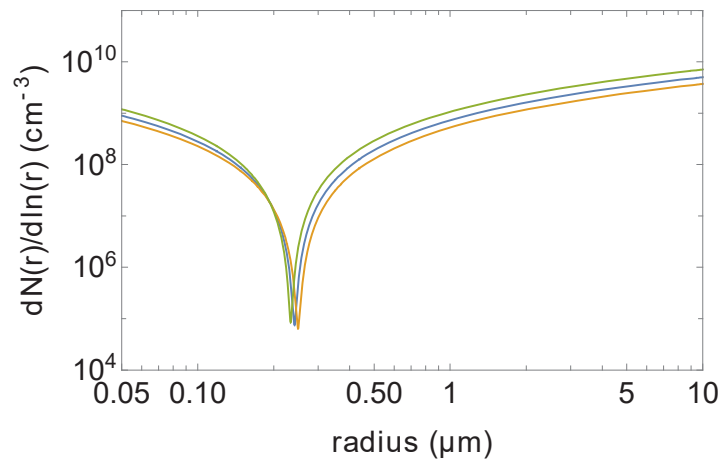

Figure 6.1
Day 166 Maximum AOD at 11:45

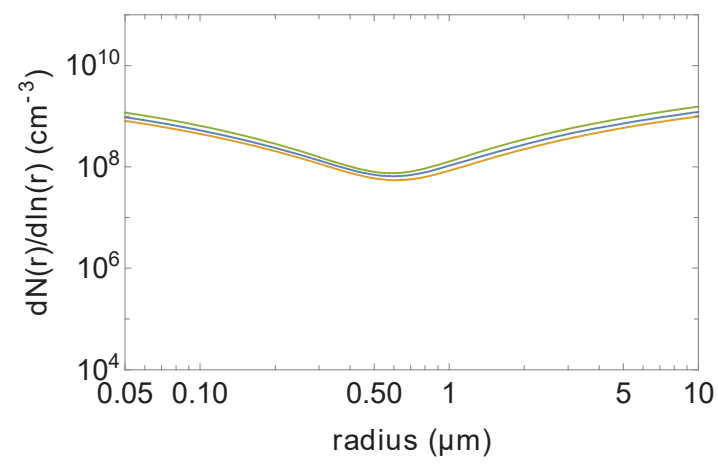

Figure 6.2

Figure 6. Bimodal Distributions from day 166 at 1000 in Figure 6.1 and at 1145 in Figure 6.2. Shading indicates uncertainty in distribution parameters.

\begin{tabular}{|c|c|c|c|c|c|c|c|}
\hline & $\begin{array}{c}\overline{\chi^{2}} \\
\text { average }\end{array}$ & $\begin{array}{c}\mathrm{N}_{0} \pm \mathrm{SDOM} \\
\left(\mathrm{cm}^{-3}\right)\end{array}$ & $\begin{array}{c}\alpha_{0} \pm \\
\text { SDOM } \\
(\mu \mathrm{m})\end{array}$ & $\begin{array}{c}\beta_{0} \pm \\
\mathrm{SDOM}\end{array}$ & $\begin{array}{c}\mathrm{N}_{1} \pm \\
\mathrm{SDOM} \\
\left(\mathrm{cm}^{-3}\right)\end{array}$ & $\begin{array}{c}\alpha_{1} \pm \text { SDOM } \\
(\mu \mathrm{m})\end{array}$ & $\begin{array}{c}\beta_{1} \pm \\
\text { SDOM }\end{array}$ \\
\hline $\begin{array}{c}166-1000 \\
\text { (Figure 6.1) }\end{array}$ & .03 & $\begin{array}{c}5.5 \times 10^{6} \pm \\
5 \times 10^{5}\end{array}$ & $\begin{array}{l}.242 \pm \\
8 \times 10^{-3}\end{array}$ & $.15 \pm .02$ & $\begin{array}{c}1.006 \times 10^{3} \pm \\
5\end{array}$ & $1.5 \pm .3$ & $.22 \pm .02$ \\
\hline $\begin{array}{c}166-1145 \\
\text { (Figure 6.2) }\end{array}$ & .008 & $\begin{array}{c}1.0 \times 10^{6} \pm \\
2 \times 10^{5}\end{array}$ & $.40 \pm .03$ & $.13 \pm .01$ & $\begin{array}{c}6 \times 10^{5} \pm \\
1 \times 10^{5}\end{array}$ & $1.9 \pm .5$ & $.16 \pm .04$ \\
\hline
\end{tabular}

Table 1. Data table containing mean fit parameter and standard deviations from day 166's Bimodal Distributions in Figure 6. The Aitken mode parameters $\mathrm{N}_{0}$, $\alpha_{0}$, and $\beta_{0}$ between 10:00 and 11:45 show the number density decreasing, the radius increasing and the width of distribution remaining constant suggesting that coalescence of the smaller particles occurs.

Day 210 Minimum AOD at 10:02

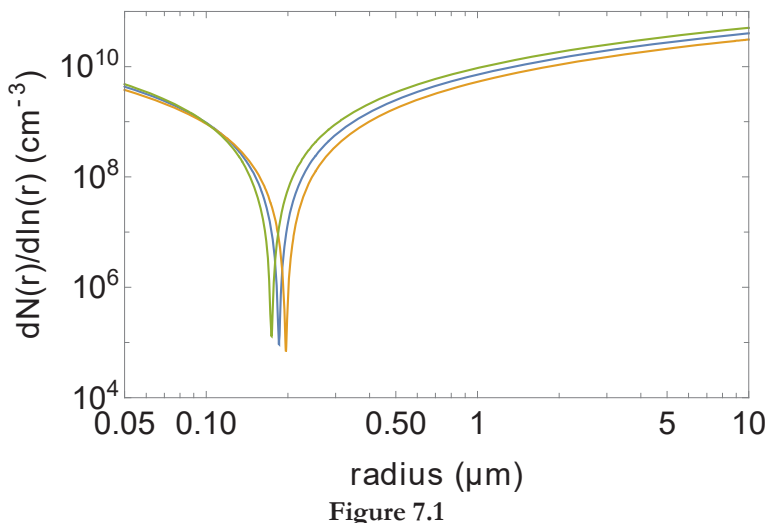

Day 210 Maximum AOD at 12:55

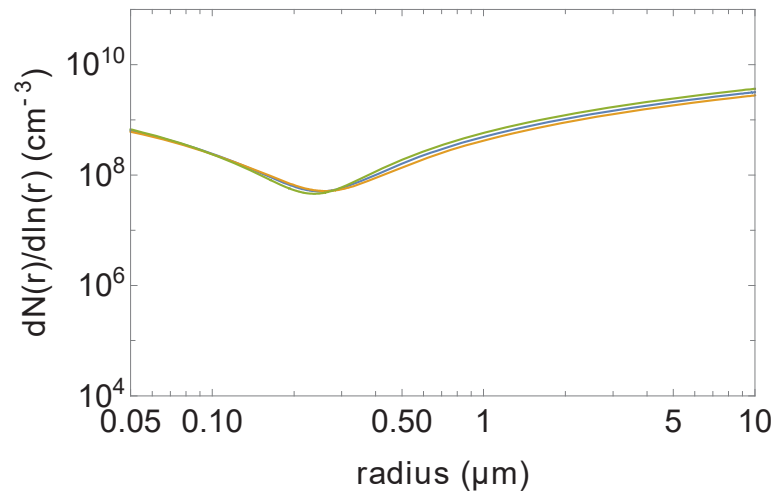

Figure 7.2

Figure 7. Bimodal Distributions from day 210 at 1002 (minimum AOD) in Figure 7.1 and at 1255 (maximum AOD) in Figure 7.2. Shading indicates uncertainty in distribution parameters.

\begin{tabular}{|c|c|c|c|c|c|c|c|}
\hline & $\begin{array}{c}\overline{\chi^{2}} \\
\text { average }\end{array}$ & $\begin{array}{c}\mathrm{N}_{0} \pm \mathrm{SDOM} \\
\left(\mathrm{cm}^{-3}\right)\end{array}$ & $\begin{array}{c}\alpha_{0} \pm \text { SDOM } \\
(\mu \mathrm{m})\end{array}$ & $\begin{array}{c}\beta_{0} \pm \\
\mathrm{SDOM}\end{array}$ & $\begin{array}{c}\mathrm{N}_{1} \pm \mathrm{SDOM} \\
\left(\mathrm{cm}^{-3}\right)\end{array}$ & $\begin{array}{c}\alpha_{1} \pm \text { SDOM } \\
(\mu \mathrm{m})\end{array}$ & $\begin{array}{c}\beta_{1} \pm \\
\text { SDOM }\end{array}$ \\
\hline $\begin{array}{c}210-1002 \\
\text { (Figure 7.1) }\end{array}$ & .007 & $\begin{array}{c}6 \times 10^{7} \pm \\
2 \times 10^{7}\end{array}$ & $.19 \pm .01$ & $.18 \pm .03$ & $1.03 \times 10^{3} \pm 30$ & $2.6 \pm .5$ & $.26 \pm .04$ \\
\hline $\begin{array}{c}210-1255 \\
\text { (Figure } 7.2 \text { ) }\end{array}$ & .001 & $\begin{array}{c}5.0 \times 10^{7} \pm \\
5 \times 10^{6}\end{array}$ & $.227 \pm .008$ & $.38 \pm .03$ & $\begin{array}{c}8 \times 10^{5} \pm \\
3 \times 10^{5}\end{array}$ & $2.5 \pm .4$ & $.28 \pm .03$ \\
\hline
\end{tabular}

Table 2. Data table containing mean fit parameter and standard deviations from day 210's Bimodal Distributions in Figure 7. 
Day 257 Minimum AOD at 12:22

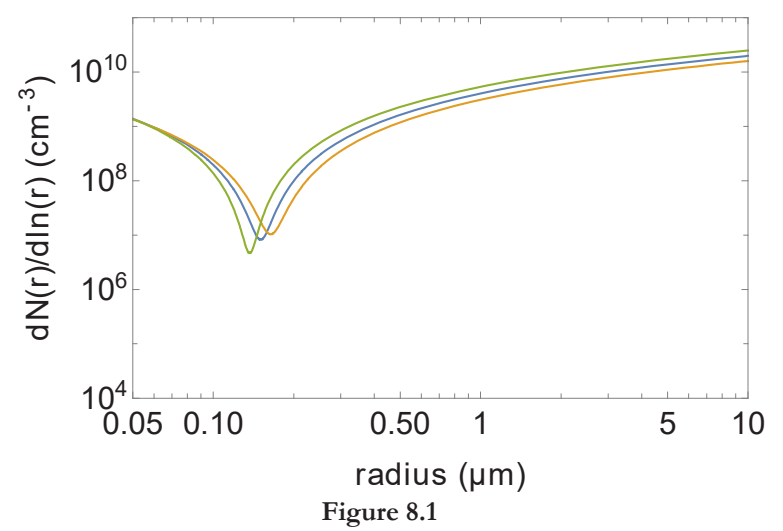

Day 257 Maximum AOD at 10:37

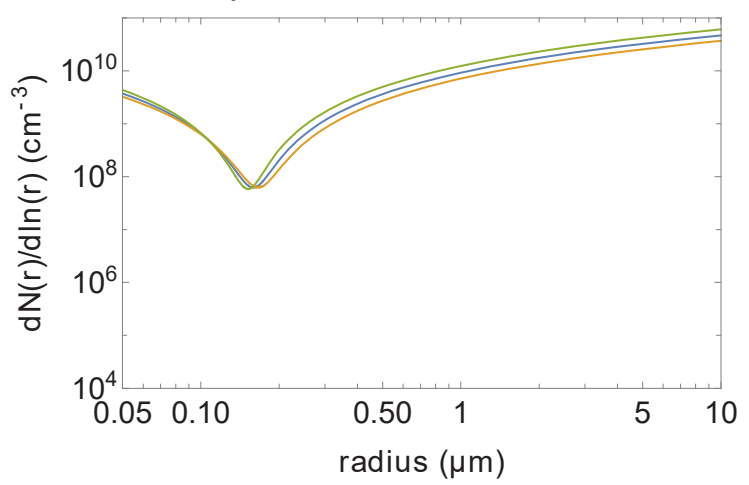

Figure 8.2

Figure 8. Bimodal Distributions from day 257 at 1222 (minimum AOD) in Figure 8.1 and at 1037 (maximum AOD) in Figure 8.2 . Shading indicates uncertainty in distribution parameters.

\begin{tabular}{|c|c|c|c|c|c|c|c|}
\hline & $\begin{array}{c}\overline{\chi^{2}} \\
\text { average }\end{array}$ & $\begin{array}{c}\mathrm{N}_{0} \pm \mathrm{SDOM} \\
\left(\mathrm{cm}^{-3}\right)\end{array}$ & $\begin{array}{c}\alpha_{0} \pm \text { SDOM } \\
(\mu \mathrm{m})\end{array}$ & $\begin{array}{c}\beta_{0} \pm \\
\text { SDOM }\end{array}$ & $\begin{array}{c}\mathrm{N}_{1} \pm \mathrm{SDOM} \\
\left(\mathrm{cm}^{-3}\right)\end{array}$ & $\begin{array}{c}\alpha_{1} \pm \text { SDOM } \\
(\mu \mathrm{m})\end{array}$ & $\begin{array}{c}\beta_{1} \pm \\
\text { SDOM }\end{array}$ \\
\hline $\begin{array}{c}257-1222 \\
\text { (Figure 8.1) }\end{array}$ & .002 & $\begin{array}{c}1.6 \times 10^{8} \pm \\
3 \times 10^{7}\end{array}$ & $.15 \pm .01$ & $.33 \pm .04$ & $\begin{array}{c}1.2 \times 10^{5} \pm \\
6 \times 10^{4}\end{array}$ & $2.2 \pm .4$ & $.29 \pm .03$ \\
\hline $\begin{array}{c}257-1037 \\
\text { (Figure 8.2) }\end{array}$ & .0009 & $\begin{array}{c}1.6 \times 10^{8} \pm \\
2 \times 10^{7}\end{array}$ & $.158 \pm .007$ & $.24 \pm .03$ & $\begin{array}{c}9 \times 10^{5} \pm \\
2 \times 10^{5}\end{array}$ & $2.5 \pm .4$ & $.30 \pm .03$ \\
\hline
\end{tabular}

Table 3. Data table containing mean fit parameter and standard deviations from day 257's Bimodal Distributions in Figure 8.

\section{CONCLUSIONS}

The forest fire season of 2017 in BC emitted large amounts of atmospheric aerosols that dispersed over much of North America. ${ }^{19}$ The synthesis of satellite imagery, backward air mass trajectories, and upper air maps all aided in understanding the findings of aerosol optical depth calculations and particle size distributions generated from solar spectrometer data in Central Minnesota. Three specific days in the summer of 2017 that correspond to times before, during, and after the BC forest fires were selected based on the atmospheric conditions, meteorological patterns, and air mass trajectories present. Data collected on these days was used to calculate aerosol optical depths which could be input into a PBPSO algorithm in order to generate particle number size distributions that illustrate how the aerosol load above Central Minnesota changed during the fires.

The primary causes for uncertainty in the results come from the fact that aerosol emissions have very different lifetimes of suspension, range in dispersion, and composition based on a wide range of factors including emission type, environmental setting, and atmospheric conditions. The aerosols emitted by these forest fires are assumed to be primary particulate matter (PPM) with a relatively high injection altitude based on the findings of Hodzic et al. facilitating a potential for long-range transport while acknowledging that aging and secondary formation could occur during the transport. The characteristics of previously studied forest fire aerosols correlate to the likelihood of the particles from the 2017 BC fires bearing similar optical and physical properties as well as the likelihood of being suspended in the upper atmosphere while passing over Central Minnesota. ${ }^{25}$

These aerosol size distributions show the variations in the number of particles in each mode. $\mathrm{N}_{1}$ values are seen to steadily increase from each day's minimum to maximum AOD, whereas $\mathrm{N}_{0}$ remains constant when smoke is likely present. In contrast, analyzing the data across the three days, $\mathrm{N}_{0}$ steadily increases while $\mathrm{N}_{1}$ remains constant excluding the minimum AOD on day 257 when there was likely some cloud cover compared to the other days experiencing relatively clear skies. Additionally, Aitken mode parameter values from day 166 to 210 to 257 show a decreasing median radius combined with an increasing number of particles in accordance with the understanding that forest fires emit a majority of aerosols with radii $<1 \mu \mathrm{m} .{ }^{25} \mathrm{During}$ the periods of clean air, variations in AOD appear to be induced by both particle sizes and number concentrations. In contrast, during periods of smoke presence, only moderate shifts in size distribution were observed while number concentrations of small particles increased by an order of magnitude or more.

Comparing the size distributions across each of the daily minima AOD, the parameters show that across all three days in the Aitken mode, the number of particles increases while the median radius decreases. Additionally, from day 210 to 257 , the width of the Aitken mode distribution increases, and the number of particles in the accumulation mode increase. These patterns suggest 
that even with varying cloud cover as indicated by accumulation mode parameters the solar spectrometer can collect accurate data about the number of Aitken mode particles as well as their median radius and width of distribution.

The generated size distributions across each of the daily maxima AOD showed different trends in particle radii, number densities, and width of distributions, but some of these anomalies are likely due to the sensor collecting data during moderate cloud cover. The major outcomes of the fit parameters from day 166 to 210 to 257 show that in the Aitken mode, the number of particles increases and the median radius decreases while in the accumulation mode, the number of particles and the median radius remains constant within uncertainties across all three days while the width of distribution increased from day 166 to 210.

In terms of future work in this field, data from terrestrial solar spectrometers can be analyzed through the PBPSO algorithm in order to generate bimodal lognormal size distributions that give valuable insights into the Aitken mode number distribution, median radius, and width of distribution even when the algorithm experiences difficulty precisely determining accumulation mode parameters under heavy aerosol loads or cloud cover.

\section{ACKNOWLEDGMENTS}

The authors thank the College of Saint Benedict and Saint John's University for the opportunity to conduct research in outstanding facilities and for their generous grant that made this work possible.

\section{REFERENCES}

1. Prijith, S., Rao, P., Mohan, M., Sai, M. and Ramana, M. (2018) Trends of absorption, scattering and total aerosol optical depths over India and surrounding oceanic regions from satellite observations: role of local production, transport and atmospheric dynamics, Environ Sci Pollut Res. https:/ / doi.org/10.1007/s11356-018-2032-0

2. Baron, P. (2010) Generation and behavior of airborne particles (aerosols), Centers for Disease Control and Prevention, https:// wmw.cdc.gov/niosh/topics/aerosols/pdfs/_Aerosol_101.pdf(accessed Jul 2018)

3. Yue, D., Hu, M., Wu, Z., Wang, Z., Guo, S., Wehner, B., Nowak, A., Achert, P., Wiedensohler, A., Jung, J., Kim, Y. and Liu, S. (2009) Characteristics of aerosol size distributions and new particle formation in the summer in Beijing, J. Geophys. Res., 114. bttps:// doi.org/10.1029/2008JD010894

4. Ramachandran, S., Srivastava, R., Kedia, S., and Rajesh, T.A. (2012) Contribution of natural and anthropogenic aerosols to optical properties and radiative effects over an urban location. Environ Res Lett 7, 034028. https://doi.org/10.1088/17489326/7/3/034028

5. Poschl, U. (2005) Atmospheric aerosols: composition, transformation, climate and health effects, Agnew Chem Int Ed Engl 44, 7520-7540. https:// doi.org/10.1002/anie.200501122

6. Mao, J. and Li, J. (2015) Retrieval of particle size distributions from aerosol optical thickness using an improved particle swarm optimization algorithm, Opt Rev 22, 809-818. https://doi.org/10.1007/s10043-015-0130-5

7. Li, S., M. J. Garay, L. Chen, E. Rees, and Y. Liu (2013), Comparison of GEOS-Chem aerosol optical depth with AERONET and MISR data over the contiguous United States, J Geophys Res Atmos 118, 11,228-11,241. https://doi.org/10.1002/jgrd.50867

8. London, J. (1985) The Observed Distribution of Atmospheric Ozone and Its Variations, in Ozone in the Free Atmosphere (Whitten, R. C. and Prasad, S. S., Eds.), 11-80, Van Nostrand Reinhold, NY.

9. U.S. Department of Energy (DOE)/National Renewable Energy Laboratory (NREL)/Alliance for Sustainable Energy, LLC (ALLIANCE). "Solar Spectra: Standard Air Mass Zero," E490_00a_AM0.xls spreadsheet, http:// rredc.nrel.gov/solar/spectra/am0/ASTM2000.html (accessed Jul 2018)

10. Bodhaine, B. A., Wood, N. B., Dutton, E. G., and Slusser, J. R. (1999) On Rayleigh optical depth calculations, J Atmos Ocean Tech 16, 1854-1861. https:// doi.org/10.1175/1520-0426(1999)016<1854:ORODC>2.0.CO;2

11. DuPaul, K.M. and Whitten, A.T. (2017) Determination of Fitted Size Distribution for Atmospheric Aerosols, Am J Undergrad Res 14(2), 105-115. bttps:// doi.org/10.33697/ajur.2017.018

12. Knapp, K. R. (2008) Scientific data stewardship of International Satellite Cloud Climatology Project B1 global geostationary observations, J Applied Remote Sensing 2, 023548. https:/ / doi.org/10.1117/1.3043461

13. Stein, A.F., Draxler, R.R, Rolph, G.D., Stunder, B.J.B., Cohen, M.D., and Ngan, F. (2015) NOAA's HYSPLIT atmospheric transport and dispersion modeling system, Bull Amer Meteor Soc 96, 2059-2077. https://doi.org/10.1175/BAMS-D-14-00110.1

14. Knapp, K. R., Frouin, R., Kondragunta, S. and Prados, A. (2005) Toward aerosol optical depth retrievals over land from GOES visible radiances: determining surface reflectance, Int J Remote Sensing, 26:18, 4097-4116. bttps:// doi.org/10.1080/01431160500099329

15. Niemi, J. V., Tervahattu, H., Vehkamaki, H., Martikainen, J., Laakso, L., Kulmala, M., Aarnio, P., Koskentalo, T., Sillan, M., Makkonen, U. (2005) Characterization of aerosol particle episodes in Finland caused by wildfires in Eastern Europe, Atmos Chem Phys 5, 2299-2310. bttps:// doi.org/ 10.5194/acp-5-2299-2005

16. Chin, M., Diehl, T., Ginoux, P., Malm, W. (2007) Intercontinental transport of pollution and dust aerosols: implications for regional air quality, Atmos Chem Phys 7, 5501-5517. bttps:/ / doi.org/10.5194/ acp-7-5501-2007 
17. Marley, N. and Gaffney, J. (2005) Introduction to Urban Aerosols and Their Impacts, ACS Symposium Series, American Chemical Society, Washington, DC, https://pubs.acs.org/doi/pdf/10.1021/bk-2006-0919.ch001 (accessed Jul 2018)

18. British Columbia Wildfire Service (2017) Wildfire Season Summary, BC Wildfire Service, bttps:// www2.gov.bc.ca/gov/content/safety/wildfire-status/about-bows/wildfire-bistory/wildfire-season-summary (accessed Jul 2018)

19. Ciolfe, T. (2017) B.C. wildfires set record high greenhouse gas emission levels, Maclean's, https:// www.macleans.ca/ news/ canada/ b-c-wildfires-set-record-high-greenhouse-gas-emission-levels/ (accessed Aug 2018)

20. Levy, R. and Hsu, C. (2015) MODIS Atmosphere L2 Aerosol Product, NASA MODIS Adaptive Processing System, Goddard Space Flight Center, USA, http://dx.doi.org/10.5067/MODIS/MOD04_L2.006 (Terra) and bttp://dx.doi.org/10.5067/MODIS/MYD04_L2.006 (Aqua) (accessed Aug 2018)

21. Tans, P., (2018) Trends in Atmospheric Carbon Dioxide, NOAA/ESRL, www.esrl.noaa.gov/gmd/crgg/trends/ (accessed Jul 2018)

22. Govett, M. (2018) NOAA/ESRL Radiosonde Database, https://ruc.noaa.gov/raobs/ (accessed Jul 2018)

23. GAW Report No. 227 (2016), WMO/GAW Aerosol Measurement Procedures, Guideline, and Recommendations, $2^{\text {nd }}$ Edition, World Meteorological Organization, Switzerland.

24. NOAA/National Weather Service (2018) Storm Prediction Center Surface and Upper Air Maps, NOAA's National Weather Service Storm Prediction Center, https:// wmw.spc.noaa.gov/obswx/maps/ (accessed Jul 2018)

25. Hodzic, A., Madronich, S., Bohn, B., Massie, S., Menut, L. and Wiedinmyer, C. (2007) Wildfire particulate matter in Europe during summer 2003:mesoscalee modeling of smoke emissions, transpor, and radiative effects, Atmos Chem Phys 7, $4043-4064$. bttps: / / doi.org/ 10.5194/acp-7-4043-2007

\section{ABOUT STUDENT AUTHOR}

Dane Kuhr is from Lakeville, Minnesota and will graduate in May 2020 with a B.A. in Applied Physics and an emphasis on PreEngineering. After spending the summer before his Junior year researching atmospheric aerosols, Dane will be developing models of atmospheric attenuation in concentrating solar power (CSP) systems at the National Renewable Energy Laboratory in Golden, $\mathrm{CO}$ before applying to graduate programs in Mechanical Engineering next year. His passion for serving others motivates him to work towards sustainable solutions in the renewable energy sector.

\section{PRESS SUMMARY}

Aerosol optical depths (AODs) were calculated from solar irradiance values collected by a Kipp-Zonen PGS-100 solar spectrometer. These AODs collected throughout 2017 were then compared to satellite imagery, upper air chart, and wind patterns to determine when air masses moved from the British Columbia region towards Central Minnesota during the BC forest fires. The AOD data from three selected days (20170615, 20170729 and 20170914) was input into a parameter based particle swarm optimization (PBPSO) algorithm to generate particle number size distributions. The generated bimodal lognormal size distributions at each of the three selected day's maxima and minima AOD illustrate an increase in the number of smaller particles with radii in the Aitken mode during the forest fires compared to the distributions before the emission of forest fire particles. The results of these number size distributions verify that the data from ground-based solar spectrometers can be used in the PBPSO algorithm to detect variations in the number, radius, and width of the distribution of particles in the Aitken mode during events like forest fires. 


\title{
Diol Mediated Tautomerization of Glycine: a DFT Study
}

\author{
Francis Sub*, Vanessa Rivera*, \& Ruben D. Parra \\ ${ }^{a}$ Department of Chemistry and Biochemistry, DePaul University, Chicago, IL 60614 \\ https:// doi.org/10.33697/ ajur.2019.014 \\ *Students:francisyjsub@gmail.com,vanessamarie124@gmail.com \\ Mentor:rparra1@depaul.edu
}

\begin{abstract}
The tautomerization of glycine via a triple proton transfer was investigated both in the gas phase and in aqueous solution using the B3LYP/6-31+G(d,p) level of theory. Fully optimized complexes of the neutral and zwitterion forms of glycine with 1,3propanediol were used for the reactant and product forms, respectively. The hydroxyl groups in the diol are conveniently oriented for glycine tautomerization through a concerted triple proton transfer facilitated by a network of three hydrogen bonds: $\mathrm{N}$ H...O-H...O-H...O=C. The activation energy for the zwitterion $\rightarrow$ neutral process increases in solution. Also, the diol-glycine complex favors the neutral over the zwitterion form in a vacuum, but the opposite is true in solution. For comparative purposes, the tautomerization of glycine via a three-proton transfer mediated by two molecules of water was also examined. The results are qualitatively similar, albeit with activation energies that are smaller to those found in the corresponding diol-mediated tautomerization.
\end{abstract}

\section{KEYWORDS}

Glycine; zwitterion, diol-mediated tautomerization; water-mediated tautomerization

\section{INTRODUCTION}

Amino acids occur predominantly in a neutral form in the gas phase and as a zwitterion in aqueous solution. ${ }^{1}$ The neutral and zwitterion forms are examples of constitutional isomers that differ in the location of a proton (i.e., tautomers). The interconversion between tautomers, also known as tautomerization, has been the subject of intense experimental and theoretical work. Being the simplest amino acid, glycine has been often used as a model system for tautomerization research. ${ }^{2-14}$ For example, theoretical calculations confirm the notion that the zwitterion form of glycine is not a minimum on the potential energy surface. However, it has also been found that two water molecules can make the zwitterion a local minimum. ${ }^{9}$ Most theoretical computations have focused on the direct intramolecular proton transfer step between the zwitterion and the neutral form, although some studies have examined the role of discrete water molecules to assist in the proton transfer. In particular, the waterassisted tautomerization in di-hydrated glycine has been reported. ${ }^{3,8}$ The notion of a water bridge to facilitate proton transfer has been applied as a convenient model to study proton transfer in biological proton pumps. ${ }^{15}$ Interestingly, polyols have also been found to facilitate long-range proton transfer. Specifically, polyols have been designed to possess a quasi-linear hydrogen-bonded network along their rigid and hydrophobic rod scaffold that facilitates transmembrane transport of protons. ${ }^{16,17}$ Moreover, polyols are also known to play a stabilizing role in proteins. ${ }^{18}$ To our knowledge, there has been neither theoretical nor experimental work on the proton transfer between glycine and a diol molecule. Because amino acids are the building blocks of peptides and hence proteins, this study is in part motivated to gain insight on the ability of a polyol to stabilize an amino acid in its neutral as well as in its zwitterion form. It is also of fundamental interest to examine the ability of the polyol to serve as a conduit for proton transfer leading to the transition from the zwitterion to the neutral amino acid. Given their relatively small sizes, glycine and 1,3propanediol were chosen as model systems for the calculations. It is worth noting that the 1,3-polyol system is a frequently occurring structural motif found in many biologically active natural products. ${ }^{19}$

In this work, the ability of a 1,3-propanediol to facilitate the tautomerization of glycine in the gas phase and the aqueous solution is investigated through density functional theory calculations. The results are compared with the equivalent tautomerization mediated by two water molecules. 


\section{COMPUTATIONAL METHODS}

All calculations were performed with the Gaussian 16 package. ${ }^{20}$ Structures were fully optimized with the B3LYP/6-31+G(d,p) method. Aqueous solution was modeled with the default method in Gaussian, i.e., SCRF= IEFPCM. Frequency calculations confirmed the nature of any optimized structure as either a minimum (all real frequencies) or a transition state (one imaginary frequency). Intrinsic reaction coordinate (IRC) calculations were performed to confirm that the transition states connect the intended minima. Complexation energies were corrected for basis set superposition error (BSSE) using the counterpoise method. ${ }^{21}$

\section{RESULTS AND DISCUSSION}

The optimized structures for the diol-mediated glycine tautomerization in the gas phase are illustrated in Figure 1. The appearances of the resulting geometries in solution are similar to their gas phase counterparts and hence are not shown. Inspection of Figure 1 reveals that the glycine-diol dimers are stabilized primarily by a sequence of three hydrogen bonds involving the two $\mathrm{OH}$ groups in the diol, the nitrogen and one of the oxygen atoms in glycine. Interestingly, the conformation adopted by glycine in the dimers is such that the oxygen atom in glycine farther from the nitrogen center is the one participating in the $\mathrm{H}$ bond. Previous theoretical and experimental work has consistently demonstrated that the zwitterion does not exist in the gas phase. However, the three $\mathrm{H}$-bonds $\mathrm{N}_{8}-\mathrm{H}_{3} \cdots \mathrm{O}_{16}-\mathrm{H}_{14} \cdots \mathrm{O}_{15}-\mathrm{H}_{13} \cdots \mathrm{O}_{2}=\mathrm{C}_{1}$ in the dimer appear to be strong enough to stabilize the glycine in its zwitterion form. Indeed, the relatively short H-bond distances (Table 1) and quasilinear angles (Table 2) confirm the presence of strong $\mathrm{H}$ bonds.

Moreover, the zwitterion is stabilized by an intramolecular $\mathrm{H}$ bond, $\mathrm{N}_{8}-\mathrm{H}_{10} \cdots \mathrm{O}_{4}=\mathrm{C}_{1}$, with the closer carboxylate oxygen. The calculated BSSE-corrected interaction energy for the zwitterion-diol dimer $(\Delta \mathrm{E}=-25.13 \mathrm{kcal} / \mathrm{mol})$ demonstrates the strength of the $\mathrm{H}$ bond interactions responsible for the stabilization of the zwitterion in the gas phase. A triple proton transfer leads to the dimer of neutral glycine and the diol molecule, neutral-diol dimer, as shown in Figure 1. H bond distances listed in Table 1 suggest somewhat weaker $\mathrm{H}$ bonds than found in the zwitterion-diol dimer. For example, the intramolecular $\mathrm{N}-\mathrm{H} \cdots \mathrm{O}=\mathrm{C}$ appears weaker in the neutral-diol dimer, with an $\mathrm{H}$ bond distance $(2.457 \AA)$ much longer than that in the zwitterion-diol dimer $(1.737 \AA)$. The weaker $\mathrm{H}$ bond strengths are confirmed by the smaller magnitude of the calculated BSSE-corrected interaction energy for the neutral-diol dimer $(\Delta \mathrm{E}=-19.28 \mathrm{kcal} / \mathrm{mol})$. Although geometry optimization in aqueous solution does not change the overall appearances of the dimers, it does bring about some noticeable changes in the $\mathrm{H}$ bond distances. Specifically, the zwitterion-diol dimer in solution exhibits longer $\mathrm{H}$ bond distances, except for the $\mathrm{O}_{15}-\mathrm{H}_{13} \cdots \mathrm{O}_{2}=\mathrm{C}_{1}$. In contrast, the neutral-diol dimer in solution exhibits shorter $\mathrm{H}$ bond distances. The intramolecular $\mathrm{N}_{8}-\mathrm{H}_{10} \cdots \mathrm{O}_{4}=\mathrm{C}_{1}$ bond in aqueous solution is elongated to $2.151 \AA$ and 2.781 $\AA$ in the zwitterion-diol and neutral-diol dimers, respectively.
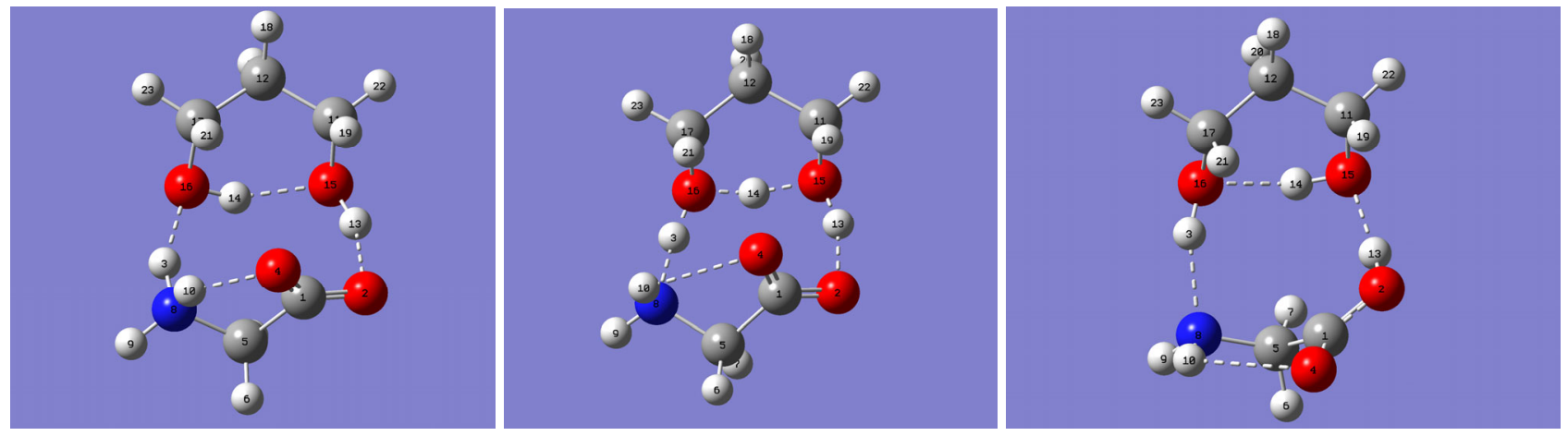

Figure 1. B3LYP/6-31+G(d,p) optimized geometries for the zwitterion-diol (left), transition state (middle), and neutral-diol (right) complexes in the gas phase.

Diol Complexes (Gas Phase)

\begin{tabular}{ccccccc}
\hline Complex & $\mathbf{N}_{\mathbf{8}}-\mathbf{H}_{\mathbf{3}}$ & $\mathbf{H}_{\mathbf{3}} \cdots \mathbf{O}_{16}$ & $\mathbf{O}_{16}-\mathbf{H}_{14}$ & $\mathbf{H}_{\mathbf{1 4}} \cdots \mathbf{O}_{15}$ & $\mathbf{O}_{15}-\mathbf{H}_{13}$ & $\mathbf{H}_{13} \cdots \mathbf{O}_{2}$ \\
\hline Zwitterion & 1.053 & 1.743 & 0.987 & 1.782 & 0.984 & 1.844 \\
Neutral & 1.866 & 0.990 & 1.816 & 0.982 & 1.729 & 0.997 \\
TS & 1.492 & 1.092 & 1.157 & 1.278 & 1.038 & 1.506
\end{tabular}


Diol Complexes (Aqueous Solution)

\begin{tabular}{ccccccc}
\hline Complex & $\mathbf{N}_{8}-\mathbf{H}_{3}$ & $\mathbf{H}_{3} \cdots \mathbf{O}_{16}$ & $\mathbf{O}_{16}-\mathbf{H}_{14}$ & $\mathbf{H}_{14} \cdots \mathbf{O}_{15}$ & $\mathbf{O}_{15}-\mathbf{H}_{13}$ & $\mathbf{H}_{13} \cdots \mathbf{O}_{2}$ \\
\hline Zwitterion & 1.049 & 1.789 & 0.982 & 1.828 & 0.984 & 1.820 \\
Neutral & 1.809 & 0.996 & 1.796 & 0.985 & 1.650 & 1.007 \\
TS & 1.501 & 1.080 & 1.217 & 1.200 & 1.067 & 1.415
\end{tabular}

Water Complexes (Gas Phase)

\begin{tabular}{ccccccc}
\hline Complex & $\mathbf{N}_{8}-\mathbf{H}_{3}$ & $\mathbf{H}_{3} \cdots \mathbf{O}_{16}$ & $\mathbf{O}_{16}-\mathbf{H}_{14}$ & $\mathbf{H}_{14} \cdots \mathbf{O}_{15}$ & $\mathbf{O}_{15}-\mathbf{H}_{13}$ & $\mathbf{H}_{13} \cdots \mathbf{O}_{2}$ \\
\hline Zwitterion & 1.046 & 1.771 & 0.993 & 1.735 & 0.997 & 1.716 \\
Neutral & 1.811 & 0.998 & 1.755 & 0.990 & 1.731 & 0.993 \\
TS & 1.200 & 1.318 & 1.175 & 1.252 & 1.226 & 1.193
\end{tabular}

Water Complexes (Aqueous Solution)

\begin{tabular}{ccccccc}
\hline Complex & $\mathbf{N}_{8}-\mathbf{H}_{3}$ & $\mathbf{H}_{3} \cdots \mathbf{O}_{16}$ & $\mathbf{O}_{16}-\mathbf{H}_{14}$ & $\mathbf{H}_{14} \cdots \mathbf{O}_{15}$ & $\mathbf{O}_{15}-\mathbf{H}_{13}$ & $\mathbf{H}_{13} \cdots \mathbf{O}_{2}$ \\
\hline Zwitterion & 1.047 & 1.774 & 0.981 & 1.775 & 0.990 & 1.770 \\
Neutral & 1.787 & 1.002 & 1.727 & 0.993 & 1.632 & 1.006 \\
TS & 1.420 & 1.122 & 1.205 & 1.213 & 1.105 & 1.339
\end{tabular}

Table 1. Relevant bond distances $(\AA)$ for the glycine-diol and glycine-(water) 2 complexes in the gas phase and solution.

Optimized geometries of each of the glycine tautomers with two water molecules in the gas phase are shown in Figure 2. It should be noted that the optimized complexes of glycine with the two molecules of water resemble closely those reported previously by other researchers using DFT and reactive force field molecular dynamics simulations (ReaxFF MD). As with the glycine-diol dimers, the glycine-(water) 2 geometries in aqueous solutions are very similar to their gas phase counterparts.

Inspection of Figures 1 and 2 reveals the striking similarity of the diol-glycine dimers and the corresponding glycine-(water) $)_{2}$ complexes. Moreover, an inspection of Table 1 and $\mathbf{2}$ shows that the structural changes in solution relative to the gas phase in these complexes are qualitatively similar to those previously noted in the glycine-diol dimers. The structural similarities of the dimers in the gas phase can be further corroborated by the BSSE-interaction energies of the zwitterion- $(\text { water })_{2}(-27.65 \mathrm{kcal} / \mathrm{mol})$ and the neutral-(water $)_{2}(-21.89 \mathrm{kcal} / \mathrm{mol})$ which compare fairly well with those of the zwitterion-diol $(-25.13 \mathrm{kcal} / \mathrm{mol})$ and the neutral-diol $(-19.28 \mathrm{kcal} / \mathrm{mol})$ discussed before. It is important to note that the two molecules of water in the complexes were effectively considered as one unit to calculate interaction energies. Thus, the two water molecules in the complex were taken as one unit, and the glycine (zwitterion or neutral) as the other unit.
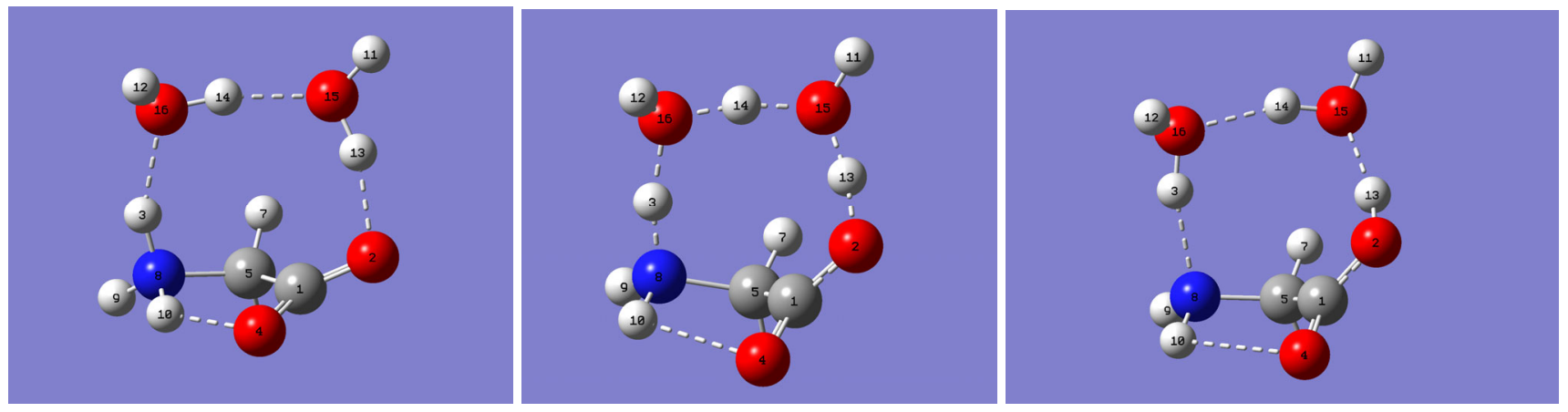

Figure 2. B3LYP/6-31+G(d,p) optimized geometries for the zwitterion-(water $)_{2}$ (left), transition state (middle), and neutral-(water) 2 (right) complexes in the gas phase. 
Diol Complexes (Gas Phase)

\begin{tabular}{ccccc}
\hline Complex & $\mathbf{N}_{8}-\mathbf{H}_{3}-\mathbf{O}_{16}$ & $\mathbf{H}_{3}-\mathbf{O}_{16} \cdots \mathbf{H}_{15}-\mathbf{O}_{15}$ & $\mathbf{O}_{15}-\mathbf{H}_{3} \cdots \mathbf{O}_{2}=\mathbf{C}_{1}$ & $\mathbf{O}_{2}=\mathbf{C}_{1}-\mathbf{C}_{5}-\mathbf{N}_{8}$ \\
\hline Zwitterion & 156.8 & 150.3 & 156.2 & -154.2 \\
Neutral & 158.2 & 147.9 & 159.9 & -126.5 \\
TS & 171.7 & 161.4 & 159.8 & -131.6
\end{tabular}

Diol Complexes (Aqueous Solution)

\begin{tabular}{ccccc}
\hline Complex & $\mathbf{N}_{8}-\mathbf{H}_{3}-\mathbf{O}_{16}$ & $\mathbf{H}_{3}-\mathbf{O}_{16} \cdots \mathbf{H}_{15}-\mathbf{O}_{15}$ & $\mathbf{O}_{15}-\mathbf{H}_{3} \cdots \mathbf{O}_{2}=\mathbf{C}_{1}$ & $\mathbf{O}_{2}=\mathbf{C}_{1}-\mathbf{C}_{5}-\mathbf{N}_{8}$ \\
\hline Zwitterion & 159.5 & 149.3 & 164.0 & -146.7 \\
Neutral & 163.0 & 148.6 & 165.8 & -104.2 \\
TS & 167.2 & 162.2 & 170.9 & -110.0
\end{tabular}

Water Complexes (Gas Phase)

\begin{tabular}{ccccc}
\hline Complex & $\mathbf{N}_{8}-\mathbf{H}_{3}-\mathbf{O}_{16}$ & $\mathbf{H}_{3}-\mathbf{O}_{16} \cdots \mathbf{H}_{15}-\mathbf{O}_{15}$ & $\mathbf{O}_{15}-\mathbf{H}_{3} \cdots \mathbf{O}_{2}=\mathbf{C}_{1}$ & $\mathbf{O}_{2}=\mathbf{C}_{1}-\mathbf{C}_{5}-\mathbf{N}_{8}$ \\
\hline Zwitterion & 156.1 & 167.5 & 163.4 & -151.3 \\
Neutral & 163.5 & 161.5 & 161.5 & -123.7 \\
TS & 166.0 & 167.7 & 169.3 & -121.5
\end{tabular}

Water Complexes (Aqueous Solution)

\begin{tabular}{ccccc}
\hline Complex & $\mathbf{N}_{8}-\mathbf{H}_{3}-\mathbf{O}_{16}$ & $\mathbf{H}_{3}-\mathbf{O}_{16} \cdots \mathbf{H}_{15}-\mathbf{O}_{15}$ & $\mathbf{O}_{15}-\mathbf{H}_{3} \cdots \mathbf{O}_{2}=\mathbf{C}_{1}$ & $\mathbf{O}_{2}=\mathbf{C}_{1}-\mathbf{C}_{5}-\mathbf{N}_{8}$ \\
\hline Zwitterion & 159.1 & 168.1 & 166.6 & -147.0 \\
Neutral & 167.2 & 161.4 & 173.0 & -96.4 \\
TS & 170.5 & 169.0 & 173.7 & -99.8
\end{tabular}

Table 2. Relevant bond angles (degrees) for the glycine-diol and glycine-(water) 2 complexes in the gas phase and solution.

As shown in Figures 1 and 2, the zwitterion-neutral tautomerization assisted by 1-3-propanediol or two molecules of water proceeds through a concerted triple proton transfer reaction. The relative electronic energies with zero-point energy corrections, $\Delta \mathrm{E}_{\mathrm{o}}$, are listed in Table 3 for both the gas phase and the aqueous solution reactions. In the gas phase, the complexes containing the neutral tautomer are favored. In solution, however, the zwitterion-containing complexes are energetically more stable. The larger stability of the zwitterion complexes can be traced down to stronger solute-solvent stabilizing interactions. Although the zwitterion carries no net charge, it is, however, a highly polar molecule. Indeed, it is found that in the gas phase the dipole moment of the zwitterion-diol complex, for example, is almost twice that of the neutral-diol complex (6.46 D vs $3.41 \mathrm{D})$, with similar results for the glycine-(water) $)_{2}$ complexes. The large difference in dipole moments is in line with the larger charge separation in the zwitterion, and it also suggests that in a polar solvent, the zwitterion-complex would be stabilized more than the neutral complex counterpart. Reaction enthalpies, $\Delta \mathrm{H}$, and reaction free energies, $\Delta \mathrm{G}$, listed in Table 3 are relatively close to each other and with $\Delta \mathrm{E}_{\mathrm{o}}$. Also listed in Table 3 are the corresponding activation energies. Relative to the gas phase, the activation energies in solution are found to be consistently higher. Thus, the solvent stabilization of the zwitterion-complex appears to be larger than the corresponding stabilization of the transition-state complex (in either the diol- or water-assisted tautomerization). The result appears consistent with the larger dipole moment of the zwitterion-complex when compared with that of the pertinent transition state. For example, the dipole moment for the transition state in the diol-complex is $5.75 \mathrm{D}$, somewhat smaller than the zwitterion-diol complex $(6.46 \mathrm{D})$. In addition to the difference in dipole moments, some structural changes occur for the transition state geometries that may in part contribute to the noted increase in activation energy upon solvation. In particular, the intramolecular $\mathrm{N}-\mathrm{H} \cdots \mathrm{O}=\mathrm{C}$ interaction appears to weaken upon solvation. Specifically, the intramolecular $\mathrm{N}_{8}-\mathrm{H}_{10} \cdots \mathrm{O}_{4}=\mathrm{C}_{1}$ bond in aqueous solution is elongated to $2.657 \AA$ from the gas phase value of $2.386 \AA$ in the transition state of the diol-containing complexes. This change is also reflected on the 20 degrees change in the $\mathrm{O}_{2}=\mathrm{C}_{1}-\mathrm{C}_{5}-\mathrm{N}_{8}$ dihedral angle (see Table 2). Likewise, the intramolecular $\mathrm{N}_{8}-\mathrm{H}_{10} \cdots \mathrm{O}_{4}=\mathrm{C}_{1}$ bond in aqueous solution is elongated to $2.803 \AA$ from the gas phase value of $2.343 \AA$ in the transition state of the water-containing complexes. 
Diol-Assisted Tautomerization

\begin{tabular}{lcccccc}
\hline Medium & $\boldsymbol{\Delta} \mathbf{E o}$ & $\boldsymbol{\Delta} \mathbf{H}$ & $\boldsymbol{\Delta} \mathbf{G}$ & $\boldsymbol{\Delta} \mathbf{E \mathbf { o } ^ { \ddagger }}$ & $\boldsymbol{\Delta} \mathbf{H}^{\ddagger}$ & $\boldsymbol{\Delta} \mathbf{G}^{\ddagger}$ \\
\hline Gas & -5.90 & -5.79 & -5.99 & 5.21 & 4.46 & 6.51 \\
Phase & 3.29 & 3.17 & 3.60 & 8.35 & 7.51 & 9.57 \\
Aqueous & & & & & &
\end{tabular}

Water-Assisted Tautomerization

\begin{tabular}{lcccccc}
\hline Medium & $\boldsymbol{\Delta E \mathbf { E }}$ & $\boldsymbol{\Delta} \mathbf{H}$ & $\boldsymbol{\Delta} \mathbf{G}$ & $\boldsymbol{\Delta} \mathbf{E o}^{\ddagger}$ & $\boldsymbol{\Delta} \mathbf{H}^{\ddagger}$ & $\Delta \mathbf{G}^{\ddagger}$ \\
\hline Gas & -7.12 & -7.05 & -7.27 & 3.62 & 2.53 & 4.94 \\
Phase & 2.44 & 2.26 & 2.59 & 7.49 & 6.31 & 8.75 \\
Aqueous & & & & &
\end{tabular}

Table 3. Reaction electronic energies with zero-point corrections $\left(\Delta \mathrm{E}_{0}\right)$, enthalpies $(\Delta \mathrm{H})$ and free energies $(\Delta \mathrm{G})$ at $298 \mathrm{~K}$ and corresponding activation energies for the glycine tautomerization reactions. Values in $\mathrm{kcal} / \mathrm{mol}$.

Although no previous work has been reported on diol-assisted tautomerization of glycine, the results presented here agree, at least qualitatively, with previous reports that show the neutral form of glycine to be the dominant species in the gas phase, while the zwitterion form to be the preferred one in solution. For example, titration studies have suggested that the free energy of the zwitterion is $7.3 \mathrm{kcal} / \mathrm{mol}$ lower than its neutral counterpart in an aqueous solution. ${ }^{14}$ Moreover, the zwitterion has been reported to be unstable in the gas phase and to transform itself into the neutral form without any energy barrier. Some gas phase calculations suggest that the complex of zwitterion with two discrete water molecules is still higher in energy $(\Delta \mathrm{E}=11.3 \mathrm{kcal} / \mathrm{mol}$ at the MP2//HF/6-31++G(d,p) level, and without zero-point energy corrections) than the corresponding most stable complex of the neutral tautomer. ${ }^{10}$ The relative order is reversed upon inclusion of solvent effects through the Polarized Continuum Model, PCM, with the zwitterion complex lying $4.4 \mathrm{kcal} / \mathrm{mol}$ below the neutral complex. It should be noted that the zwitterion complex with two water molecules has essentially the same geometrical orientation as the one considered here. However, the most stable neutral complex (reference 10) has the two water molecules forming a ring with the carboxylic acid end of glycine.

In addition to relative energies, the free energy of activation has also been extensively studied. For example, experimental results indicate that the zwitterion-neutral tautomerization in glycine has a free energy of activation of $14.4 \mathrm{kcal} / \mathrm{mol} .^{22} \mathrm{Computational}$ studies provide different activation energies depending on the methods used or the mechanistic approach employed. An activation free energy of $5.42 \mathrm{kcal} / \mathrm{mol}$ has been reported for a direct intramolecular zwitterion-neutral proton transfer using B3LYP/6-31++G(d,p) and the IEFPCM model to account for solvent (aqueous) effects. ${ }^{6}$ Molecular dynamics simulations, on the other hand, have yielded activation energies of about $11.6 \mathrm{kcal} / \mathrm{mol},{ }^{5} 12.7 \mathrm{kcal} / \mathrm{mol},{ }^{7}$ and about $17.0 \mathrm{kcal} / \mathrm{mol} ., 14$ An assisted triple proton transfer mechanism in aqueous solution considering two molecules of water has also been investigated. An activation energy of $15.8 \mathrm{kcal} / \mathrm{mol}$ has been estimated through molecular dynamics simulations using a reactive force field developed to describe proton transfer in glycine. ${ }^{3}$

\section{CONCLUSIONS}

To the best of the authors' knowledge, the first study on the diol-assisted tautomerization of glycine is reported in this study. Calculations were performed at the B3LYP/6-31+G(d,p) level of theory in the gas phase and aqueous solution using the IEFPCM method. Dimer complexes between 1,3-propanediol and glycine (zwitterion and neutral form) were chosen as model systems. Strong hydrogen bonds stabilize the complexes, especially the complex of the zwitterion form of glycine. The mechanistic pathway investigated consisted of a triple proton transfer facilitated by the two OH groups from the diol acting as a bridge between the carboxyl and the amine group. The same mechanistic pathway was examined considering two molecules of water instead of the diol molecule. The results for both diol-mediated and water-mediated tautomerization appear comparable to one another and in qualitative agreement with other relevant experimental and theoretical studies. The results of this study should serve as motivation for exploring further the role that a diol, and in general a polyol, may play in tautomerization and other proton transfer reactions. Additional studies need to be conducted to address some of the limitations inherent to this study. For example, the results presented in this work refer to the static geometries of the optimized complexes, and it should be therefore a subject of future work to examine the dynamics of the interactions, for example by using ab initio molecular dynamics simulations. It is also desirable to consider other density functional methods that can better account for dispersion corrections, such as the APFD functional. Alternatively, the use of correlated methods such as MP2 or CCSD should also be explored along with correlationconsistent basis sets. Because of the impractical cost of computational methods that can account for both static and dynamic 
electron correlation, a good compromise between computational cost and accurate computation of thermochemical quantities is usually offered by composite methods such as the CBS-QB3 method, and therefore future studies should consider such composite method to improve upon the predicted results presented in this work. Although the default method for modeling chemistry in solution in the Gaussian 16 package was used in this study, it is nonetheless desirable that other approaches be considered. For example, the SDM method has been found to perform well for predicting reaction energies in solution. ${ }^{23}$ Future studies should also consider explicitly the conformational space of both the diol and glycine/zwitterion to account for the energy cost, if any, in adopting the particular conformations adopted by the molecules in the reactions both in the gas phase and in solution. Although the concerted nature of the multiple-proton transfer was noted in this study, additional studies need to be carried out to determine whether the transfer is synchronous or asynchronous. For this purpose, obtaining the reaction force and force constant profiles along the intrinsic reaction coordinate, or IRC would prove useful. ${ }^{24}$ Furthermore, although no intermediate species was found in the transition from zwitterion-complex to neutral-complex rendering the reaction to be concerted, it is still subject for future work whether this remains so upon using higher levels of theory. Presumably, intermediates species would need to be stabilized enough to be detected computationally. It might also be possible that increasing the polyol chain would give rise to intermediate species. Indeed, it has been reported that a single charge center in polyol chains can be significantly stabilized by the hydrogen-bonded network, and it can also enhance the interaction energy between adjacent noncharged $\mathrm{OH}$ groups in the network. ${ }^{25}$ The results suggest the intriguing possibility that a sequential rather than a concerted proton transfer may occur under these conditions. Thus, using higher polyol chains, namely a triol or tetraol to assist glycine (and other amino acids) tautomerization, will be considered in our group and the results published elsewhere.

\section{ACKNOWLEDGMENTS}

The authors thank the Department of Chemistry and Biochemistry of DePaul University for support.

\section{REFERENCES}

1. Schmidt, J., and Kass, S. R. (2013) Zwitterion vs Neutral Structures of Amino Acids Stabilized by a Negatively Charged Site: Infrared Photodissociation and Computations of Proline-Chloride Anion, J. Phys. Chem. A 117, 4863-4869. bttps://pubs.acs.org/doi/abs/10.1021/jp402267c

2. Kim, C. K., Park, B-H., Lee, H. W., and Kim, C. K. (2013) Comprehensive Studies on the Tautomerization of Glycine: A Theoretical Study, Org. Biomol. Chem. 11 14007-1413. bttps://pubs.rsc.org/en/content/articlelanding/2013/ob/c2ob26602d

3. Rahaman, O., van Duin, A. C. T., Goddard III, W. A., and Doren, D. J. (2011) Development of a ReaxFF Reactive Force Field for Glycine and Application to Solvent Effect and Tautomerization, J. Phys. Chem. B. 115, $249-261$. bttps://pubs.acs.org/doi/abs/10.1021/jp108642r

4. Tang, W., Cai, C., Zhao, S., and Liu, H. (2018) Development of Reaction Density Functional Theory and Its Application to Glycine Tautomerization Reaction in Aqueous Solution, J. Phys. Chem. C. 122, 20745-20754. https://pubs.acs.org/doi/abs/10.1021/acs.jpcc.8605383

5. Tolosa, S., Hidalgo, A., and Sansón, J. A. (2012) Amino Acid Tautomerization Reactions in Aqueous Solution via Concerted and Assisted Mechanisms Using Free Energy Curves from MD Simulation, J. Phys. Chem. B. 116, 13033-13044. https://pubs.acs.org/doi/abs/10.1021/jp307391s

6. Tuñon, I., Silla, E., and Ruiz-Lopéz, M. F. (2000) On the Tautomerization Process of Glycine in Aqueous Solution, Chem. Phys. Lett. 321, 433-437. https:// doi.org/10.1016/S0009-2614(00)00365-1

7. Leung, K., and Rempe, B. S. (2005) Ab Initio Molecular Dynamics Study of Glycine Intramolecular Proton Transfer in Water, J. Chem. Phys. 122, 184506(1)-184506(12). https:/ / aip.scitation.org/doi/10.1063/1.1885445

8. Balta, B., and Aviyente, V. (2004) Solvent Effects on Glycine II. Water-Assisted Tautomerization, J. Comp. Chem. 25 , 690-703. bttps://doi.org/10.1002/jcc.10422

9. Jensen, J. H., and Gordon, M. S. (1995) On the Number of Water Molecules Necessary to Stabilize the Glycine Zwitterion, J. Am. Chem. Soc. 117, 8159-8170. https://pubs.acs.org/doi/abs/10.1021/ja00136a013

10. Aikens C. M., and Gordon, M. S. (2006) Incremental Solvation of Nonionized and Zwitterionic Glycine, J. Am. Chem. Soc. 128, 12835-12850. https://pubs.acs.org/doi/10.1021/ja062842p

11. Fernández-Ramos, A., Smedarchina, Z., Siebrand, W., and Zgierski, M. Z. (2000) A Direct-Dynamics Study of the Zwitterionto-Neutral Interconversion of Glycine in Aqueous Solution, J. Chem. Phys. 113, 9714-9721. https://doi.org/10.1063/1.1322084

12. Pérez de Tudela, R., and Marx, D. (2016) Water-Induced Zwitterionization of Glycine:Stabilization Mechanism and Spectral Signatures, J. Phys. Chem. Lett 7, 5137-5142. https://pubs.acs.org/doi/full/10.1021/acs.jpclett.6b02247

13. Wada, G., Tamura, E., Okina, M., and Nakamura, M.(1982) On the Ratio of Zwitterion Form to Uncharged Form of Glycine at Equilibrium in Various Aqueous Media, Bull. Chem, Soc. Jpn. 55, 3064-3067. https:// doi.org/10.1246/bcsj.55.3064

14. Okuyama-Yoshida, N., Nagaoka, M., and Yamabe, T. (1998) Origin of the Transition State on the Free Energy Surface: Intramolecular Proton Transfer Reaction of Glycine in Aqueous Solution, J. Phys. Chem. 102, 285-292.

https://pubs.acs.org/doi/abs/10.1021/jp982534g 
15. Konig, P. H., Ghosh, N., Hoffmann, M., Elstner, M., Tajkhorshid, E., Frauenheim, Th., and Cui, Q. (2006) Toward theoretical analysis of long-range proton transfer kinetics in biomolecular pumps, J. Phys. Chem. A 110, 548-563. bttps://pubs.acs.org/doi/10.1021/jp052328q

16. Weiss, L. A., Sakai, N., Ghebremariam, B., Ni, C., and Matile S. (1997) Rigid Rod-Shaped Polyols: Functional Nonpeptide Models for Transmembrane Proton Channels J. Am. Chem. Soc. 119, 12142-12149. bttps://pubs.acs.org/doi/abs/10.1021/ja973126d

17. Sakai, N., Mareda, J., and Matile S. (2005) Rigid-Rod Molecules in Biomembrane Models: From Hydrogen-Bonded Chains to Synthetic Multifunctional Pores. Acc. Chem. Res. 38, 79-87. https://pubs.acs.org/doi/abs/10.1021/ar0400802

18. Gekko, K. (1981) Enthalpy and entropy of transfer of amino acids and diglycine from water to aqueous polyol solutions $J$. Biochem. 90, 1643-1652. https:// doi.org/10.1093/oxfordjournals.jbchem.a133639

19. Iwata, M., Yazaki, R., Suzuki, Y., Kumagai, N., and Shibasaki, M. (2009) Direct catalytic asymmetric aldol reactions of thioamides: Toward a stereocontrolled synthesis of 1,3-Polyols. J. Am. Chem. Soc. 131, 18244-18245. bttps://pubs.acs.org/doi/10.1021/ja909758e

20. Frisch, M. J., Trucks, G.W., Schlegel, H.B., et al. (2016) Gaussian 16, revision B.01. Gaussian, Inc.: Wallingford, CT. https://gaussian.com/citation/

21. Boys, S. F., and Bernardi, F. (1970) The Calculation of Small Molecular Interactions by the Differences of Separate Total Energies. Some Procedures with Reduced Errors, Mol. Phys. 19, 553-566. bttps:/ / www.tandfonline.com/doi/abs/10.1080/00268977000101561

22. Slifkin, M.A., and Ali, S.M. (1984) Thermodynamic Parameters of the Activation of Glycine Zwitterion Protonation Reactions, J. Mol. Liq. 4, 215-221. https:/ / doi.org/10.1016/0167-7322(84)80025-2

23. Marenich, A. V., Cramer, C. J., and Truhlar, D. G. (2009) Universal-solvation model based on solute electron density and on a continuum model of the solvent defined by the bulk dielectric constant and atomic surface tensions, J. Phys. Chem. B, 113, 6378-6396. https://pubs.acs.org/doi/abs/10.1021/jp810292n

24. Yepes, J. S. Murray, P. Politzer, P. Jaque, (2012) The reaction force constant: an indicator of the synchronicity in double proton transfer reactions, Phys. Chem. Chem. Phys. 14, 11125-11134. https://pubs.rsc.org/en/content/articlelanding/2012/cp/c2cp41064h

25. Shokri, A., Schmidt, J., Wang, X-B., and Kass, S. R. (2012) Hydrogen Bonded Arrays: The power of multiple hydrogen bonds, J. Am. Chem. Soc. 134, 2094-2099. https://pubs.acs.org/doi/abs/10.1021/ja2081907

\section{ABOUT STUDENT AUTHORS}

Francis Suh will graduate in 2019 with a Bachelors of Science degree in Chemistry and intends to further her studies by pursuing a Ph.D. in chemistry. She plans to start her Ph.D. program in Organic Chemistry in the fall of 2019, at the University of Illinois - at Chicago. Vanessa Rivera graduated in 2018 with a Bachelors of Science degree in Chemistry and is currently obtaining a Doctorate in Pharmacy at Rosalind Franklin University of Medicine and Science.

\section{PRESS SUMMARY}

To our knowledge, there has been neither theoretical nor experimental work on the proton transfer between glycine and a diol molecule. The tautomerization of glycine via a triple proton transfer was investigated both in the gas phase and in aqueous solution using computational studies. It has been shown that the hydrogen bond from the glycine facilitates the proton transfer between glycine and a diol molecule. It was also shown for the diol-glycine complex to favor the neutral over the zwitterion form in a vacuum, but the opposite is true in solution. 


\title{
Psychological Constructs as Predictors of Strength Gains in a Strength Training Course
}

\author{
Kathrine Hadley \&o Joanna Morrissey \\ Department of Kinesiology, California State University Monterey Bay, Seaside, CA \\ https://doi.org/10.33697/ajur.2019.015 \\ Student:kphadley@usc.edu \\ Mentor:jmorrissey@csumb.edu
}

\begin{abstract}
Strength training has many benefits, both affective and physical health-related. However, little research has been done on the psychological constructs that play an important role in exercise adherence, maintenance, and outcomes regarding strength training specifically. The purpose of this study was to examine self-efficacy (SE), perceived competence (PC), and outcome expectancy (OE), several of the key psychological constructs, as predictors of strength gains in a strength training course. It was hypothesized that the changes in participants' measures of SE, PC, and OE from baseline to post-training would predict participants' actual strength gains, but not the levels of SE, PC, and OE at baseline and post-training independently. Participants $(n=20 ; 50 \% 20-21$ years old, 40\% 22-24 years old, 10\% 25 years or older; $60 \%$ female, $40 \%$ male; $45 \%$ Caucasian, 30\% Hispanic/Latino, 20\% multiracial, 5\% Asian/Pacific Islander) in a 15-week strength training technique (STT) course completed a battery of psychological questionnaires assessing SE, PC, and OE in addition to fitness tests consisting of a vertical jump test, an estimated one repetition maximum bench press and a back squat at baseline, mid- and post-training. One-way repeated measures ANOVA was used to examine differences in SE, PC, and OE at baseline, mid-, and post-training. Spearman correlation and multiple regression analyses were used to determine the predictive specificity of baseline, mid-, post-training levels, and changes in SE, PC, and OE on strength gains. ANOVA results show a significant time effect, as there was a significant increase in all three variables over time, suggesting that course participation increased students' SE, OE, and PC about strength training. Baseline scores, posttraining scores, and changes in SE, PC, and OE were not significant predictors of changes in strength or power scores. These results suggest that while an instructor-led STT course may increase SE, OE, and PC for individuals with varying strength training experience and positively influence college students' well-being, these psychological constructs may not predict strength gains. Future research should examine possible predictive factors for strength training outcomes in larger, more heterogeneous populations.
\end{abstract}

\section{KEYWORDS}

Strength Training; Self-Efficacy; Perceived Competence; Outcome Expectancy; Psychological Constructs; Kinesiology; Strength and Conditioning; Resistance Training

\section{INTRODUCTION}

In recent years, resistance training has been emphasized as a necessary part of a regular exercise program as its benefits have become more apparent. One form of resistance training is strength training which refers to a "systematic program of exercises designed to increase an individual's ability to exert or resist force". ${ }^{1}$ Strength training has similar benefits for people of all ages and is an important component of a regular physical activity program. ${ }^{1} \mathrm{~A}$ variety of benefits arise from consistently practiced resistance training: reduction in loss of muscle mass and strength, improved bone strength, hormone regulation and production associated with age, management of chronic diseases, increased ease of daily activities, decreased sports-related injuries in youth, and improvements in mental health. ${ }^{1,2}$ Due to the challenging nature of many physical exercises, psychological constructs play a crucial role in exercise initiation and adherence and, thus, in the physical and psychological benefits of physical activity. ${ }^{2}$ Selfefficacy (SE), perceived competence (PC), and outcome expectancy (OE) are three of these constructs that have been shown to influence physical activity and exercise as well as overall well-being. ${ }^{2,3,4}$ Exercise SE has been shown to predict perceived wellness and wellness subscales of physical, spiritual, intellectual, psychological, and emotional dimensions, indicating that exercise may improve overall well-being that extends beyond the physical health benefits. ${ }^{4}$

While many studies focus solely on one psychological construct, the purpose of combining all three in this study was to identify their potential individual and combined influence on exercise outcomes and to examine further how they might work together. Furthermore, by measuring all three, the impact of exercise on each construct over time can be compared relative to one another as well as together. 


\section{Literature Review}

Self-Efficacy

Self-efficacy, originating from Bandura's social-cognitive theory, is one of the most well-known and studied psychological constructs. ${ }^{3} \mathrm{SE}$ is an individual's belief in oneself to execute situation-specific behavior and enhances an individual's behavioral persistence. ${ }^{2,3} \mathrm{SE}$ is comprised of four components: performance accomplishments, vicarious experience, verbal persuasion, and emotional arousal. ${ }^{3}$ Performance accomplishments are built on personal mastery experiences, where repeated success increases, and consistent failure lowers those mastery expectations. Due to its nature, performance accomplishment is influenced by the timing, pattern, and outcome of the experiences, where initial failure may decrease the likelihood of behavioral persistence. Vicarious experience refers to observing others' successes in a difficult situation which can enhance one's confidence in his/her abilities to complete the same task. Verbal persuasion refers to the suggestive power of others to convince a person that they can overcome past failures or fears, and while it is much easier to obtain than the other components of SE, it is not quite as effective. ${ }^{3}$ Finally, emotional arousal, wherein an individual judges his/her capability by one's physiological state in a high-stress situation, serves as a source of information regarding personal competency and can influence future performance (e.g., anxiety may hinder performance).

SE has been studied extensively as it relates to physical activity and exercise; however, little research has been done examining SE as it relates to strength training in particular. A study examining the relationship of SE and physical activity in a population of sedentary older women participating in a 12-week high-intensity strength training program found that the participants' perceptions of strength and balance efficacy capabilities were associated with better performance-based and self-reported "Activities of Daily Living." These results suggest that SE may have a mediating effect on the influence of physical activity and function in older women. ${ }^{5}$ A more recent study in 2014 examined the effects of twice-a-week resistance training compared to flexibility training on postpartum women. Results showed that the resistance training group had greater strength gains and greater improvements in exercise SE than the flexibility training group. ${ }^{6}$ Another study found that participants in a 10 -week circuit weight training group had greater improvement in strength and endurance than did the volleyball control group. In addition, participants' baseline levels of SE predicted their post-test strength gains. ${ }^{7}$ Finally, another study found that a strength training intervention on college women improved their SE and psychological well-being as well as strength, endurance, and body fat. ${ }^{8}$ The research surrounding exercise suggests that SE influences the physical outcomes and benefits of exercise, including improvements in strength, daily functioning, and psychological well-being.

\section{Perceived Competence}

PC is a psychological construct from Ryan \& Deci's self-determination theory (SDT) which suggests that motivation is created by the satisfaction of three psychological needs (competence, autonomy, and relatedness) and that individuals will pursue behaviors that fulfill these needs. ${ }^{2,9}$ In SDT, PC is at the level of the individual's need for "innate psychological nutriments that are essential for ongoing psychological growth, integrity, and well-being." ${ }^{, 9}$ Unlike SE which focuses on an individual's belief in their capabilities of performing a specific behavior, PC refers specifically to beliefs surrounding meaningful behaviors that provide fulfillment of one's psychological needs. Measuring PC addresses the content and pursuit of an individual's need to master challenging and meaningful tasks that will fulfill one's need for psychological growth and personal effectance on their environment. ${ }^{2,9}$

PC in exercise has primarily been studied as it relates to general physical activity and sports, in which many studies utilize the structured class design (e.g., high school physical education classes), yet little research has focused on the role of PC specifically in strength training. In a study of 307 middle school students' enjoyment and cardiorespiratory fitness in physical education classes, regression analysis showed that PC was the only significant contributor to cardiorespiratory fitness (19.3\%) and only accounted for $4.2 \%$ of the variance in physical activity. ${ }^{10}$ These results show that students who believed they would do well in physical education obtained higher fitness levels, which suggests that PC affects fitness outcomes. In another study examining physical activity in grades $3^{\text {rd }}$ to $12^{\text {th }}$, PC had a direct negative effect on students' sedentary behavior and a positive effect on physical activity, suggesting that higher levels of PC may lead to increased physical activity. ${ }^{11} \mathrm{PC}$ also had a stronger indirect effect on physical activity at home than physical activity at school which may be associated with the formal structure of more physical activity at school (e.g., physical education classes) as compared to the more variable opportunities at home which provide more autonomy and may, therefore, lead to increased PC and attraction to physical activity. In a study of 146 women participating in community-based aerobics classes, PC was found only to have an effect on intrinsic motivation when self-determination was low. ${ }^{12}$ In other words, when individuals participated in the classes because they wanted to, their performance perceptions were irrelevant; in contrast, when participants felt they had to participate in the classes and had low PC, their intrinsic motivation was negatively affected. These findings have strong implications for individuals who are beginning an exercise program, as it is important for perceptions of competence to be fostered when their intrinsic motivation might be exceptionally low. The literature surrounding PC suggests that the construct plays a vital role in physical activity and exercise outcomes as well as motivation. 


\section{Outcome Expectancy}

OE has been referred to in literature dating back to the 1930s and is a fundamental aspect of many theories, including the theory of planned behavior, social learning theory, social cognitive theory, the theory of reasoned action, and expectancy theory. ${ }^{13} \mathrm{OE}$ is thought to play an important role in motivation and is defined as a person's belief that a specific behavior will lead to certain positive and negative consequences related to one's physical health or affective outcomes, such as impacts on one's mood or mental state. ${ }^{3,13}$ This construct differs from SE in the fact that it is not a person's belief in their ability to do the task, but rather one's belief about what will happen if they are successful at completing the task. While positive outcome expectancies (i.e., perceived benefits) promote a behavior, negative outcome expectations (e.g., soreness from exercise) and perceived barriers, circumstances preventing the behavior (e.g., the cost of joining a gym), both impede the behavior. ${ }^{13}$ When an individual perceives the benefits of the activity to outweigh the barriers, they are more likely to engage in the behavior because the payoff is worth the price.

Similar to SE, OE has been studied in general physical activity and exercise, but little research has been done to examine this construct specific to resistance or strength training. Several studies have shown a correlation between OE and initiation of physical activity but not necessarily the adherence to it. While positive OE may lead to an individual adopting a behavior, satisfaction becomes a contributor to continued participation. ${ }^{13}$ In other words, because OE does not measure actual satisfaction, it might not be as effective at predicting adherence to a behavior once the individual's subjective fulfillment becomes a factor. While the relationship between OE and SE is not clear, as the two constructs are related and often overlap, some research suggests that $\mathrm{OE}$ may predict variance in physical activity not explained by SE and other social cognitive variables. In a telephone survey study of 3,607 adults in Hawaii, researchers found that individuals who thought they needed more than recommended amounts of moderate intensity physical activity in order to gain health benefits reported more daily amounts of physical activity than those who thought they needed equal amounts or amounts less than recommended by experts. ${ }^{14}$ These results show that the individuals' beliefs in the amount of time they thought they needed to spend doing physical activity in order to receive the health benefits were related to how much time they spent doing it, suggesting that OE influences not just the adoption of physical activity but also the duration and frequency. In a study of 335 adults aged 60-95, affective OE was a stronger predictor of exercise at 6 months than physical health-related OE; furthermore, SE did not predict exercise but was a mediator via intention and affective OE. ${ }^{15}$ These findings suggest that affective OE may be a better predictor of physical activity adherence than healthrelated $\mathrm{OE}$ and $\mathrm{SE}$ in some populations. Research shows that $\mathrm{OE}$ is related to and may predict physical activity and exercise initiation and adherence in many populations across the lifespan.

In summary, previous research has examined the relationship of SE, PC, and OE in physical activity (primarily aerobic) and exercise adherence, maintenance, and performance; however, there is less research examining these constructs in strength training outcomes. Furthermore, there is a gap in the literature regarding SE, PC, and OE in college-aged populations wherein these constructs may play a role in overall health and wellness. Thus, the purpose of this study was to examine the predictive power of these psychological constructs on participants' strength gains in a strength and conditioning technique course. It was hypothesized that the changes in the participants' measures of SE, PC, and OE from baseline to post-training would predict participants' strength gains (hypothesis 1) and that the independent levels at baseline and post-training would not (hypothesis 2).

\section{METHODS AND PROCEDURES}

Design

A single group design was used. The independent variables in this study were the changes in participants' SE, PC, and OE scores from baseline to post-training, and the independent scores of these psychological constructs at baseline and post-training. The dependent variables were the changes in participants' strength and power over the 15-week period for both hypotheses. The Committee for Protection of Human Subjects (CPHS), California State University, Monterey Bay's review board for research involving human subjects approved this study (\#16-005).

\section{Participants}

The total population consisted of a convenience sample of twenty (three junior, 17 senior) kinesiology students enrolled in a 15week university "Technique in Strength and Conditioning" course. Participants identified as 60\% females, 40\% males; 45\% Caucasian, 30\% Hispanic/Latino, 20\% multiracial and 5\% Asian/Pacific Islander. Age was reported in ranges: 50\% 20-21 years old, 40\% 22-24 years old, and 10\% 25 years or older. Students also reported on their participation in strength training prior to the course (Table 1). 


\begin{tabular}{|c|c|c|}
\hline & Frequency & Percentage \\
\hline \multicolumn{3}{|l|}{ Previous ST Experience } \\
\hline Do not strength train & 2 & 10 \\
\hline$\leq 1 \mathrm{yr}$ & 6 & 30 \\
\hline $1-2 \mathrm{yr}$ & 3 & 15 \\
\hline $3-4 \mathrm{yr}$ & 7 & 35 \\
\hline $5+y r$ & 2 & 10 \\
\hline \multicolumn{3}{|l|}{ Previous ST Days/Week } \\
\hline 0 & 0 & 0 \\
\hline 1 & 4 & 20 \\
\hline 2 & 1 & 5 \\
\hline 3 & 2 & 10 \\
\hline 4 & 9 & 45 \\
\hline 5 & 2 & 10 \\
\hline 6 & 0 & 0 \\
\hline 7 & 2 & 10 \\
\hline \multicolumn{3}{|l|}{ Previous ST Min/Session } \\
\hline Do not strength train & 4 & 20 \\
\hline$\leq 20 \mathrm{~min}$ & 4 & 20 \\
\hline $21-30 \mathrm{~min}$ & 0 & 0 \\
\hline $31-45 \mathrm{~min}$ & 5 & 25 \\
\hline $46-60 \mathrm{~min}$ & 4 & 20 \\
\hline $60+\min$ & 3 & 15 \\
\hline \multicolumn{3}{|l|}{ Perceived Resistance Level } \\
\hline Do not strength train & 3 & 15 \\
\hline Light & 4 & 20 \\
\hline Moderate & 11 & 55 \\
\hline Heavy & 2 & 10 \\
\hline
\end{tabular}

Table 1. Participation in strength training prior to the course. $S T$ strength training.

Measures

An introduction to the study and first questionnaire were given to the students during the first week of class, and they were informed that their participation in the study was voluntary and would not affect their grade in the course. No incentives were offered.

\section{Questionnaires}

The demographics questionnaire was administered only at baseline and contained questions regarding sex, race/ethnicity, age, academic class status, previous strength training experience, number of days per week spent strength training, and length and perceived resistance level of strength training sessions.

Lubans, Aguiar, \& Callister's four-item SE and five item OE questionnaires were adapted to measure participants' SE and OE for strength training. ${ }^{16}$ Both questionnaires were rated on five-point Likert scales $(1=$ Strongly Disagree to $5=$ Strongly Agree).

Example SE item: "I have the strength to complete strength training exercises." Example OE item: Strength training will improve my performance in sports and other physical activities."

Ryan \& Deci's four-item PC Scale was adapted to measure participants' PC about strength training regularly and was rated on a seven-point Likert scale ( $1=$ not at all true to $7=$ very true $).{ }^{9}$ Example PC item: "I am able to meet the challenge of strength training regularly." 


\section{Physical Fitness Assessments}

Muscular strength was assessed using estimated one repetition maximum (1RM) test for bench press and back squat using an Olympic barbell. Participants were given a choice to use 3RM or 5RM load to measure estimated 1RM in which they performed 3 or 5 repetitions, respectively, of the given exercise with as much weight as possible. Muscular power was measured by the average of a three-trial vertical jump test. The sit-and-reach test determined flexibility.

\section{Procedure}

Participants were required to attend the course twice weekly for 80 minutes each session, which was held in a private gym setting on campus. Participants were divided into seven groups based on height for ease of equipment use by the instructor, a Certified Strength and Conditioning Specialist and Professor of Exercise Physiology. At the beginning of the course, participants were introduced to various strength training exercises by the instructor and were required to perform these exercises in their small groups with peer and instructor feedback. Throughout the course, the groups alternately created and taught an exercise routine to the class; all participants were then required to perform that routine utilizing proper technique under the supervision of the course instructor.

Participants completed the questionnaire at week one (baseline), week eight (mid-training), and week 15 (post-training) at the conclusion of the course. Baseline fitness levels were assessed at week four (to allow for knowledge of proper lifting technique and neural adaptations), mid-training at week nine, and post-training at week 15 using the National Strength and Conditioning Association standards: estimated 1RM for barbell bench press and barbell back squat to assess strength, vertical jump test to assess power, and sit-and-reach test to measure flexibility; however, flexibility was not used for this analysis.

\section{Statistical Analysis}

Preliminary analysis of one-way repeated measures analysis of variance (ANOVA) was used to test the effects of the strength and conditioning technique course on students' SE, PC, and OE over time. Spearman correlations were then used to assess associations of these psychological outcomes with strength and power gains. Strength gains were defined as the difference in pounds used for bench press and back squat from baseline to post-training. Power gains were defined as the difference in inches in the vertical jump from baseline to post-training. Finally, nine multiple regressions using the enter method were run to test the effect of each psychological variable (i.e., SE, PC, OE) as predictors (independent variables) on changes in strength (i.e., bench press, back squat) and power (i.e., vertical jump) scores as an outcome (dependent variables). Specifically, to test hypothesis one, the following nine multiple regressions were run: change in psychological variable scores from baseline to post-training on bench press; change in psychological variable scores from baseline to post-training on back squat; and change in psychological variable scores from baseline to post-training on vertical jump. Next, the following six multiple regressions were run to test hypothesis two: 1) psychological variable scores at baseline on bench press; 2) psychological variable scores at baseline on back squat; 3) psychological variable scores at baseline on vertical jump; 4) psychological variable test scores at post-training on bench press; 5) psychological variable test scores at post-training on back squat; and, 6) psychological variable test scores at post-training on vertical jump.

\section{Results}

Changes in Psychological Constructs

One-way repeated measures ANOVA was used to examine differences in SE, PC, and OE at baseline, mid-, and post-training. Mauchly's test indicated that the assumption of sphericity had been violated for all three domains $\left(\mathrm{SE}: \chi^{2}(2)=14.20, p=0.001\right.$, PC: $\chi^{2}(2)=11.48, p=0.003$, OE: $\left.\chi^{2}(2)=7.95, p=0.019\right)$, therefore degrees of freedom were corrected using Greenhouse-Geisser estimates of sphericity (SE: $\varepsilon=0.65$, PC: $\varepsilon=0.68$, OE: $\varepsilon=0.74)$. Results show a significant time effect, (SE: $F(1.29,24.59)=20.54$, $p<0.001$, PC: $F(1.36,25.82)=19.72, p<0.001$, OE: $F(1.47,28.00)=6.94, p<0.01)$. Follow-up comparisons indicated that each pairwise difference for SE and PC was significant, $p<0.01$, and from pre- to post- for OE, $p<0.01$. As demonstrated in Table 2 , there was a significant increase in all three variables over time, suggesting that course participation increased students' SE, PC, and $\mathrm{OE}$ about strength training.

\begin{tabular}{|c|c|c|c|c|}
\hline Measure & Pre-Test & Mid-Test & Post-Test & Pre-Post Change \\
\hline Self-Efficacy & $\begin{array}{c}15.0(4.1) \\
(\mathrm{n}=20)\end{array}$ & $\begin{array}{c}18.1(1.6) \\
(\mathrm{n}=20)\end{array}$ & $\begin{array}{c}19.5(0.8) \\
(\mathrm{n}=20)\end{array}$ & $\begin{array}{c}4.6(3.7)^{*} \\
(\mathrm{n}=20)\end{array}$ \\
\hline $\begin{array}{c}\text { Outcome } \\
\text { Expectancy }\end{array}$ & $\begin{array}{c}23.3(1.8) \\
(\mathrm{n}=20)\end{array}$ & $\begin{array}{c}24.5(1.2) \\
(\mathrm{n}=20)\end{array}$ & $\begin{array}{c}24.7(1.0) \\
(\mathrm{n}=20)\end{array}$ & $\begin{array}{l}1.4(1.7)^{*} \\
(\mathrm{n}=20)\end{array}$ \\
\hline $\begin{array}{c}\text { Perceived } \\
\text { Competence }\end{array}$ & $\begin{array}{l}20.0(6.4) \\
(n=20)\end{array}$ & $\begin{array}{c}24.9(3.0) \\
(\mathrm{n}=20)\end{array}$ & $\begin{array}{c}27.0(1.7) \\
(\mathrm{n}=20)\end{array}$ & $\begin{array}{l}7.0(5.5)^{*} \\
(\mathrm{n}=20)\end{array}$ \\
\hline
\end{tabular}

Table 2. Psychological and performance measures before, during, and after training. Values reported as mean (SD). Note: All significance values refer to within-group changes (i.e., post-test minus pre-test) evaluated by paired t-test. ${ }^{*} \mathrm{p}<0.001$. 


\section{Psychological Constructs and Strength Gains Correlations}

Spearman correlations were run on changes in levels of SE, PC, and OE and strength and power gains in bench press, back squat, and vertical jump (Table 3). Changes in the levels of these psychological constructs were not significantly correlated to strength and power gains.

\begin{tabular}{|c|c|c|c|c|}
\hline & & Squat & Bench & $\begin{array}{l}\text { Vertical } \\
\text { Jump }\end{array}$ \\
\hline Self-Efficacy & $\begin{array}{l}\mathrm{r}= \\
\operatorname{sig}= \\
\mathrm{n}=\end{array}$ & $\begin{array}{l}0.276 \\
0.302 \\
16\end{array}$ & $\begin{array}{l}-0.314 \\
0.220 \\
17\end{array}$ & $\begin{array}{l}-0.125 \\
0.622 \\
18\end{array}$ \\
\hline $\begin{array}{l}\text { Perceived } \\
\text { Competence }\end{array}$ & $\begin{array}{l}\mathrm{r}= \\
\operatorname{sig}= \\
\mathrm{n}=\end{array}$ & $\begin{array}{l}-0.121 \\
0.655 \\
16\end{array}$ & $\begin{array}{l}-0.052 \\
0.843 \\
17\end{array}$ & $\begin{array}{l}-0.199 \\
0.428 \\
18\end{array}$ \\
\hline $\begin{array}{l}\text { Outcome } \\
\text { Expectancy }\end{array}$ & $\begin{array}{l}\mathrm{r}= \\
\operatorname{sig}= \\
\mathrm{n}=\end{array}$ & $\begin{array}{l}0.189 \\
0.483 \\
16\end{array}$ & $\begin{array}{l}-0.461 \\
0.063 \\
17\end{array}$ & $\begin{array}{l}0.064 \\
0.801 \\
18\end{array}$ \\
\hline
\end{tabular}

The overall model examining the impact of changes in SE, PC, and OE (independent variables) on bench press strength gains from baseline to post-training (dependent variable) was not significant $[F(3,13)=.537, p=.665]$ and explained none of the variance in bench press scores, $R^{2}=.110$. Changes in SE $(\beta=.042, p=.918), \operatorname{PC}(\beta=-.098, p=.804)$, and $\mathrm{OE}(\beta=-.324, p=$ .254) were not significant predictors of changes in bench press scores.

The overall model examining the impact of changes in SE, PC, and OE on back squat strength gains from baseline to posttraining was not significant $[F(3,12)=.875, p=.481]$ and explained none of the variance in back squat scores, $R^{2}=.179$. Changes in $\mathrm{SE}(\beta=.524, p=.208), \mathrm{PC}(\beta=-.611, p=-.137)$, and $\mathrm{OE}(\beta=-.021, p=.939)$ were not significant predictors of changes in back squat scores.

The overall model examining the impact of changes in SE, PC, and OE on vertical jump power gains from baseline to posttraining was not significant $[F(3,14)=1.312, p=.310]$ and explained $5.2 \%$ of the variance in vertical jump scores, $R^{2}=.219$. Changes in SE $(\beta=-.038, p=.906), \operatorname{PC}(\beta=-.366, p=.256)$, and $\mathrm{OE}(\beta=.297, p=.243)$ were not significant predictors of changes in vertical jump scores.

The overall model examining the impact of baseline levels of SE, PC, and OE on bench press strength gains from baseline to post-training was not significant $[F(3,13)=.485, p=.699]$ and explained none of the variance in bench press scores, $R^{2}=.101$. Changes in SE $(\beta=.071, p=.877)$, PC $(\beta=-.002, p=.997)$, and $\mathrm{OE}(\beta=.301, p=.277)$ were not significant predictors of changes in bench press scores.

The overall model examining the impact of baseline levels of SE, PC, and OE on back squat strength gains from baseline to posttraining was not significant $[F(3,12)=.832, p=.502]$ and explained none of the variance in back squat scores, $R^{2}=.172$. Changes in SE $(\beta=-.556, p=.196), \operatorname{PC}(\beta=.602, p=.171)$, and $\mathrm{OE}(\beta=.056, p=.840)$ were not significant predictors of changes in back squat scores.

The overall model examining the impact of baseline levels of SE, PC, and OE on vertical jump power gains from baseline to posttraining was not significant $[F(3,14)=1.311, p=.310]$ and explained $5.2 \%$ of the variance in vertical jump scores, $R^{2}=.219$. Changes in SE $(\beta=-.132, p=.701), \operatorname{PC}(\beta=.527, p=.141)$, and $\mathrm{OE}(\beta=-.216, p=.381)$ were not significant predictors of changes in vertical jump scores.

The overall model examining the impact of post-training levels of SE, PC, and OE on bench press strength gains from baseline to post-training was not significant $[F(3,13)=.009, p=.999]$ and explained none of the variance in bench press scores, $R^{2}=.002$. Changes in SE $(\beta=.025, p=.938)$, PC $(\beta=.030, p=.928)$, and $\mathrm{OE}(\beta=-.015, p=.961)$ were not significant predictors of changes in bench press scores. 
The overall model examining the impact of post-training levels of SE, PC, and OE on back squat strength gains from baseline to post-training was not significant $[F(3,12)=.390, p=.762]$ and explained none of the variance in back squat scores, $R^{2}=.089$. Changes in SE $(\beta=-.187, p=.571)$, PC $(\beta=-.011, p=.973)$, and $\mathrm{OE}(\beta=.279, p=.360)$ were not significant predictors of changes in back squat scores.

The overall model examining the impact of post-training levels of SE, PC, and OE on vertical jump power gains from baseline to post-training was not significant $[F(3,14)=.497, p=.691]$ and explained none of the variance in vertical jump scores, $R^{2}=.096$. Changes in SE $(\beta=.055, p=.853), \mathrm{PC}(\beta=.259, p=.406)$, and $\mathrm{OE}(\beta=.048, p=.863)$ were not significant predictors of changes in vertical jump scores.

\section{Conclusions}

The purpose of this study was to examine the predictive power of SE, PC, and OE on strength gains in a strength and conditioning technique course. The results suggest that these psychological constructs are not correlated with and do not play a role in predicting strength or power gains. However, the results show evidence that these psychological constructs may be improved by a strength training technique course independent of strength and power gains which may be in part due to the structured class design and implementation of the four components of SE.

The first hypothesis was rejected as results found that changes in SE, PC, and OE from baseline to post-training did not predict and were not significantly correlated with strength or power gains. In concordance with the second hypothesis, the independent levels of SE, PC, and OE at baseline and post-training did not predict strength or power gains.

These results also showed that all three constructs increased significantly from baseline to post-training, while SE and PC increased significantly at each time point (i.e., baseline to mid-training, mid-training to post-training, baseline to post-training). These improvements suggest that participation in the strength and conditioning technique course increased students' SE, PC, and OE about strength training which supports previous research suggesting that a regular physical activity routine can improve exercise-related SE, PC, and OE. These findings may have implications beyond strength training specifically and impact an individual's overall attitude and belief toward exercise in general and positively affect intrinsic motivational states.

Due to the small, homogeneous sample of this study, these results may not apply to students who do not have the physiological knowledge of junior and senior kinesiology students, which may have allowed them to progress faster or otherwise impacted their physical progress or attitudes toward strength training. Furthermore, the non-significant findings may be in part due to the small sample size and the lack of a control group.

The four primary sources of SE (performance accomplishments, vicarious experience, verbal persuasion, and emotional arousal) were all utilized in the structure of the course. Participants were able to track their performance accomplishments through their strength and power output as the 15 weeks progressed. They achieved vicarious experience by watching their instructor and peers perform and teach the exercises to the class. Furthermore, encouragement and coaching regarding proper form and technique from the instructor and peers served as verbal persuasion in addition to one-on-one coaching from the instructor when necessary. Finally, experiencing and becoming adjusted to the physiological arousal of strength training accounted for the emotional arousal aspect of SE. The structure of the strength and conditioning course was conducive to improving students' SE and may be a contributing factor to the improvements in students' PC and OE as well.

These findings have substantial implications for strength and conditioning coaches, as the experience of strength training may improve SE, which has been known to increase many facets of well-being, independent of actual performance improvements. Furthermore, both the strength training experienced and inexperienced students had improvements in their SE which suggest that hands-on experience may bridge the gap between the science and practice of strength training and play a key role in helping kinesiology students become more efficacious and competent in strength training. These competencies may extend past strength training to weight-bearing exercises in general due to the similar nature regarding form, technique, physiology, and practice. These are essential traits to have surrounding weight training, considering many of these students are preparing to become coaches and trainers.

Examining SE, PC, and OE in a college population of kinesiology students in a strength and conditioning technique course was a novel study design and topic. These preliminary findings should encourage researchers to examine these constructs in larger, more heterogeneous populations with individuals who have no kinesiology education. Furthermore, given that the analyses showed no significant correlation between these psychological constructs and strength or power gains, future researchers should examine what other factors, psychological or otherwise, play a role in strength training outcomes. 


\section{Acknowledgments}

The authors thank the student research assistants that contributed to the study-Casey Gahan, Alyssa Alonzo, and Heather Mogul—the kinesiology student participants, California State University, Monterey Bay Kinesiology Department, and Dr. Ryan Luke who was the strength training course instructor.

\section{REFERENCES}

1. American College of Sports Medicine. (n.d.). ACSM | Fact Sheets. https://www.acsm.org/public-information/brochures-fact-sheets/factsheets (accessed Mar 2016)

2. Rodgers, W. M., Markland, D., Selzler, A., Murray, T. C., \& Wilson, P. M. (2014). Distinguishing perceived competence and self-efficacy: An example from exercise, Res Q Exerc Sport 85(4), 527-539. https:/ / doi.org/10.1080/02701367.2014.961050

3. Bandura, A. (1977). Self-efficacy: toward a unifying theory of behavioral change, Psychol Rev 84(2), 191-215. https:// doi.org/10.1037//0033-295x.84.2.191

4. Sidman, C. L., D'Abundo, M. L., \& Hritz, N. (2009). Exercise self-efficacy and perceived wellness among college students in a basic studies course, Int Electronic J Health Educ 12, 162-174.

5. Mihalko, S. L. (1997). Strength training in older women: Does self-efficacy mediate improvements in physical function? Ph.D. Dissertation: University of Illinois at Urbana-Champaign. http:// hdl.handle.net/2142/86410 (accessed Mar 2016)

6. LeCheminant J., Hinman T., \& Tucker L. (2014). Effect of resistance training on body composition, self-efficacy, depression, and activity in postpartum women, Scand J Med Sci Sports 24(2), 414-421. https:// doi.org/ 10.1111/j.1600-0838.2012.01490.x

7. Ewart, C. K., Stewart, K. J., Gillilan, R. E., \& Kelemen, M. H. (1986). Self-efficacy mediates strength gains during circuit weight training in men with coronary artery disease, Med Sci Sports Exerc 18(5), 531-540. https://doi.org/10.1249/00005768198610000-00007

8. Don, B. M. (1997). The effects of strength training on cardiovascular reactivity to stress and psychological well-being in college women. Ed.D. Dissertation: Boston University.

9. Deci, E. L., \& Ryan, R. M. (2000). The "what" and "why" of goal pursuits: Human needs and the self-determination of behavior, Psychol Inq 11(4), 227-268. https:/ / doi.org/10.1207/s15327965pli1104_01

10. Gao, Z. (2008). Perceived competence and enjoyment in predicting students' physical activity and cardiorespiratory fitness. Percept Mot Skills 107(6), 365-372. https:// doi.org/10.2466/pms.107.6.365-372

11. Bai, Y., Chen, S., Vazou, S., Welk, G. J., \& Schaben, J. (2015). Mediated effects of perceived competence on youth physical activity and sedentary behavior, Res Q Exerc Sport 86(4), 406-413. https:/ / doi.org/ 10.1080/02701367.2015.1087639

12. Markland, D. (1999). Self-determination moderates the effects of perceived competence on intrinsic motivation in an exercise setting, J Sport Exerc Psychology 21(1), 351-361. bttps:/ / doi.org/10.1123/jsep.21.4.351

13. Williams, D. M., Anderson, E. S., Winnet, R. A. (2005). A review of the outcome expectancy construct in physical activity research, Ann Behav Med 29(1), 70-79. bttps:// doi.org/10.1207/s15324796abm2901_10

14. Heinrich, K., Maddock, J., Bauman., A. (2011). Exploring the relationship between physical activity knowledge, health outcomes expectancies, and behavior, J Phys Act Health 8(1), 404-409. bttps:/ / doi.org/ 10.1123/jpah.8.3.404

15. Gellert, P., Ziegelmann, J. P., \& Schwarzer, R. (2012). Affective and health-related outcome expectancies for physical activity in older adults, Psychol Health 27(7), 816-828. https:/ / doi.org/10.1080/08870446.2011.607236

16. Lubans, D., Aguiar, E., \& Callister, R., (2010). The effects of free weights and elastic tubing resistance training on physical self-perception in adolescents, Psychol Sport Exerc 11(6), 497-504. https:// doi.org/ 10.1016/j.psychsport.2010.06.009

\section{ABOUT STUDENT AUTHORS}

Kathrine Hadley graduated Magna Cum Laude from California State University, Monterey Bay in May 2016 with a Bachelor of Art in Psychology, minor in Kinesiology, with an emphasis on Health \& Wellness. She has been working in the fitness industry for five years and was recently accepted to the University of Southern California's Master of Science in Nutrition, Healthspan, and Longevity. Her goal is to own private practice as a registered dietitian with a focus on a holistic approach to health through a whole food, plant-based diet, regular exercise, and stress management.

\section{PRESS SUMMARY}

Self-efficacy, perceived competence, and outcome expectancy are related to an individual's exercise adherence, maintenance, and outcomes, as well as overall well-being. The purpose of this study was to examine if the changes in these psychological constructs from baseline to post-training was predictive of actual strength gains for participants in a fifteen-week university strength training course. A battery of psychological questionnaires and strength assessments were utilized to measure these changes. While all three of these constructs improved significantly from baseline to post-training, these changes did not predict actual strength gains, indicating that the hands-on learning experience of strength training may be more influential on self-efficacy, perceived competence, and outcome expectancy than the performance improvements. These findings have substantial implications for strength training coaches and kinesiology students entering the field. 


\title{
Modeling Networks of Evolving Populations
}

\author{
Sean Elliott \\ Department of Mathematics, Southern Methodist University, Dallas, TX
}

https://doi.org/10.33697/ajur.2019.016

Student: sjelliott@smu.edu*

Mentors:dskinner@mit.edu,thagstrom@smu.edu

\begin{abstract}
The goal of this research is to devise a method of differential equation based modeling of evolution that can scale up to capture complex dynamics by enabling the inclusion of many-potentially thousands-of biological characteristics. Towards that goal, a mathematical model for evolution based on the well-established Fisher-Eigen process is built with a unique and efficient structure. The Fisher-Eigen partial differential equation (PDE) describes the evolution of a probability density function representing the distribution of a population over a phenotype space. This equation depends on the choice of a fitness function representing the likelihood of reproductive success at each point in the phenotype space. The Fisher-Eigen model has been studied analytically for simple fitness functions, but in general no analytic solution is known. Furthermore, with traditional numerical methods, the equation becomes exponentially complex to simulate as the dimensionality of the problem expands to include more phenotypes. For this research, a network model is synthesized and a set of ordinary differential equations (ODEs) is extracted based on the Fisher-Eigen PDE to describe the dynamic behavior of the system. It is demonstrated that, when juxtaposed with full numerical PDE simulations, this ODE model finds well-matched transient and precise equilibrium solutions. This prototype method makes modeling of high-dimensional data possible, allowing researchers to examine and even predict complex dynamic behavior based on a snapshot of a population.
\end{abstract}

\section{KEYWORDS}

Evolutionary Modeling; Mathematical Biology; Network Dynamics; Ordinary Differential Equations; Partial Differential Equations; Fisher-Eigen model; Phenotype; Fitness Function

\section{INTRODUCTION}

The theory of evolution by natural selection has transformed modern biology. Understanding evolution allows one to understand the origin of the genetic diversity present in the world today. With this in mind, accurate mathematical and computer models that capture the vast complexity and intricate dynamics of evolution may give researchers new insights into how evolution has occurred and may predict evolutionary events in the future. ${ }^{1}$

Such model-based tools would have many vital humanitarian uses. For instance, they could help researchers understand the dynamics of evolution of bacteria. Using the models, researchers could estimate how fast bacteria mutate from a harmless to a harmful state and also find which states are passed through along the way. With these insights, researchers could attempt to block harmful mutations. This is important because such mutations could enable antibiotic resistance, for example, which poses a growing, deadly, global threat. In addition, efficient models could help the broader field of genetics with other applications, such as studying how organisms may be impacted and propagate under different climate change conditions or other environmental disasters. These studies could aid attempts to minimize damage and to make associated public policy decisions. 
Some models of evolution have been developed in the past. ${ }^{1,2}$ The model presented here builds on previous work but with the goal of enabling the inclusion of far more-potentially thousands more-biological characteristics. We model evolution using the Fisher-Eigen strategy, also known as a Darwinian strategy, based on the work of Fisher ${ }^{3}$ and Eigen. ${ }^{2}$ Consider an arbitrary list of genetic characteristics. While each characteristic takes on discrete values, we can approximate each one as a continuous variable as long as the total number of organisms is large. ${ }^{4}$ We define a probability density function that tells us the proportion of the population with a specific value of a genetic characteristic at a specific time. The Fisher-Eigen strategy models evolutionary adaptation to the environment through a differential equation that controls this probability density function.

We then consider a fitness function, which represents the likelihood of successful reproduction of an organism given a value of the genetic characteristic for that organism. We can think of this fitness function as the negative of a potential energy function; the population tends towards a local maximum, just as for a potential energy function an object tends towards a local minimum. As an example, suppose we had a population of finches whose beaks varied in length. We can imagine that certain lengths would be optimal for certain types of food, and that there would be local maxima centered around each such length.

With a fitness function, we can model the population given the initial condition of the population at time zero using the Fisher-Eigen equation as defined in previous works. ${ }^{5,6}$ This equation includes a comparison of the local fitness at every point to the average fitness of the entire population, making it a global selection process. It also includes a diffusion term that represents random changes in the population over time.

Dunkel et al. contrasted the Fisher-Eigen strategy with the Smoluchowski process (a model for particles in Brownian motion) as a model for evolution. ${ }^{5}$ The Smoluchowski process, which comes from thermodynamics, is based on local interactions between particles and the potential, as opposed to the global selection used in the Fisher-Eigen strategy. Dunkel et al. looked at the case where the fitness function is of the form $a x^{2}-b x^{4}$ with $a, b>0$. The potential associated with such a fitness function is called a symmetric double-well potential.

Instead of computing the solutions exactly, Dunkel et al. estimated the transition rates between the two wells of the potential function using an eigenvalue analysis. They used a system of master equations, which models how a system of multiple states evolves. Each master equation describes the probability of being in a state through a differential equation involving transition rates with all of the states. In the study by Dunkel et al. there were two states, each representing a well of the potential function. There were equal transition rates between the states due to the symmetry of the fitness function. By expanding the probability density function in terms of the eigenfunctions of the Hamiltonian operator $H=-D \nabla^{2}+U$, they were able to estimate the transition rate in terms of the first two eigenvalues of $H .^{5}$

Dunkel et al. analyzed these two strategies when the fitness function was quadratic and found an exact solution for each. ${ }^{7}$ For general fitness functions, however, the equation is unsolved analytically. Furthermore, simulating the equation quickly becomes computationally infeasible as the number of phenotypes grows. In practice, one would hope to model a population that varies in hundreds or even thousands of phenotypes, but this is impossible when considering a numerical simulation of the Fisher-Eigen equation.

We model a population that evolves according to the Fisher-Eigen process but using a system of ordinary differential equations (ODEs) over a network. The motivation for constructing such a model is to simplify the modeling of highdimensional fitness landscapes and avoid needing to take data samples at different times. Pearce et al. ${ }^{8}$ introduced such a technique in the case of a Smoluchowski process. Their work proceeds as follows. First, gather data on a population at a specific time. Then, project the data onto a lower dimensional space. In this new space, find clusters of points and associate them with nodes of a network. Consider these nodes as the minima of some function analogous to a potential energy. Then, analyze the dynamics of such a network. Finding the transition rates between states is enough to reconstruct the dynamics of the population in the original landscape. This allows one to calculate quantities such as the 
average time to move from one state to another.

We perform a similar analysis on networks which behave according to the Fisher-Eigen process. This allows one to model a population which evolves according to the Fisher-Eigen process but over a high-dimensional landscape. However, studying networks is more difficult for the Fisher-Eigen process than for the Smoluchowski process because of the global selection property of the Fisher-Eigen process. In the Smoluchowski process, each individual particle behaves as it would in equilibrium because the selection process is only based on local behavior. ${ }^{5}$ In contrast, for the Fisher-Eigen process an individual organism behaves differently depending on whether or not the entire population is in an equilibrium state. However, if we measure the population at equilibrium, we can determine the fitness landscape, which we can then use to study the dynamics of the population, provided we can analyze the dynamics of an arbitrary fitness landscape.

In the ODE model presented here, the domain is split into 3 regions. The integrals over each region are modeled using a system of ODEs. To derive these ODEs the flux between regions is computed and is used to calculate the transition rates between nodes. The purpose of this method of finding transition rates is to demonstrate that there exist transition rates that make the ODE model accurate. To implement this model in practice, however, some other technique of calculating the transition rates that does not rely on knowing the data at every time would be used. For example, researchers could mark population members and measure the average transition time between regions.

This paper demonstrates by simulation that data from the equilibrium state can be used to model the selection process as a system of ODEs over a network. The organization of the paper is as follows. Methods and Procedures gives a mathematical description of the problem and explains how a 2-dimensional fitness landscape was simulated and the ODE model was constructed. The outcomes are discussed in Results. Conclusions provides areas of future research and conclusions.

\section{METHODS AND PROCEDURES}

We model a phenotype as taking values $\mathbf{x}$ in $\mathbb{R}^{n}$. Let $p(\mathbf{x}, t)$ be the probability density function of the population at a time $t \in \mathbb{R}_{\geq 0}$. For a given time $t, p(\mathbf{x}, t)$ is a probability density function describing the distribution of the population over the phenotype space. As an illustrative example, we use a colony of bacteria.

We also have a fitness function $F(\mathbf{x})$ defined over the phenotype space. Roughly, this tells us the likelihood of successful reproduction for an organism with phenotype vector $\mathbf{x}$. Let $U=-F$ be the potential associated with the fitness function. Then the Fisher-Eigen equation is

$$
\frac{\partial p(\mathbf{x}, t)}{\partial t}=(\bar{U}(t)-U(\mathbf{x})) p(\mathbf{x}, t)+D \nabla^{2} p(\mathbf{x}, t)
$$

Equation 1.

where $\bar{U}(t):=\int_{\mathbb{R}^{n}} U(\mathbf{x}) p(\mathbf{x}, t) d \mathbf{x}$ is the average potential of the population at time $t$, and $D>0$ is the diffusion constant. The diffusion constant in this case is a property of the phenotype space that expresses the flux of the population through an area over time, based on a population gradient. The distance and area units of $D$ are in quantified phenotypes, which may be aggregated phenotypes as a result of dimension reduction. The time units of $D$ will be appropriate to the system under study, such as days, years, generations or lifetimes.

We simulated the Fisher-Eigen process for a specific 2-dimensional fitness landscape using MATLAB. The particular landscape was chosen so that it could be modeled as a network of three nodes while still possessing interesting dynamics; in one dimension, the three node situation is less interesting because organisms on each of the outer nodes have to pass through the middle node to get to the other side. In two dimensions, however, organisms can move around the middle node. In the case of bacteria, such a relationship between phenotypes and fitness could be present, for example, if phenotypes 1 and 2 were chemical resistance and adhesion, respectively. The highest peak would represent the phe- 
notypic region in which bacteria have sufficient adhesion to attach to a host in the environment. The optimal level of chemical resistance in the bacteria would then depend on whether or not they have such adhesive properties, forming the two smaller peaks around different levels of chemical resistance.

We selected the fitness function to be the following:

$$
\begin{aligned}
F\left(x_{1}, x_{2}\right) & =3 e^{-20\left(x_{1}^{2}+\left(x_{2}-\frac{\sqrt{3}}{2}\right)^{2}\right)}+e^{-10\left(\left(x_{1}+0.5\right)^{2}+x_{2}^{2}\right)}+e^{-10\left(\left(x_{1}-0.5\right)^{2}+x_{2}^{2}\right)}+ \\
& +\frac{1}{2} e^{-15\left(x_{1}-0.25\right)^{2}-5\left(x_{2}-\frac{\sqrt{3}}{4}\right)^{2}}+\frac{1}{2} e^{-5 x_{1}^{2}-15 x_{2}^{2}}-\frac{1}{10}\left(x_{1}^{2}+x_{2}^{2}\right) .
\end{aligned}
$$

We chose the fitness function with one global maximum and two other local maxima. This gave the highest peak at $P_{1}=\left(0, \frac{\sqrt{3}}{2}\right)$, the middle peak at $P_{2}=(0.5,0)$, and the lowest peak at $P_{3}=(-0.5,0)$. The smaller Gaussians created a less steep valley between $P_{3}$ and $P_{2}$ and between $P_{2}$ and $P_{1}$. The last term was added so that the boundary of the space would have a low fitness. The population starts out at the lowest peak and moves toward the highest one. Some bacteria move towards the highest peak directly but are slowed down because of the steepness of the highest peak. However, by first moving to the middle peak, the bacteria can move to the highest one more easily. Which of these paths is optimal depends on the parameters in the fitness function and is reflected in the transition rates between peaks. The fitness function is shown graphically in Figure 1.

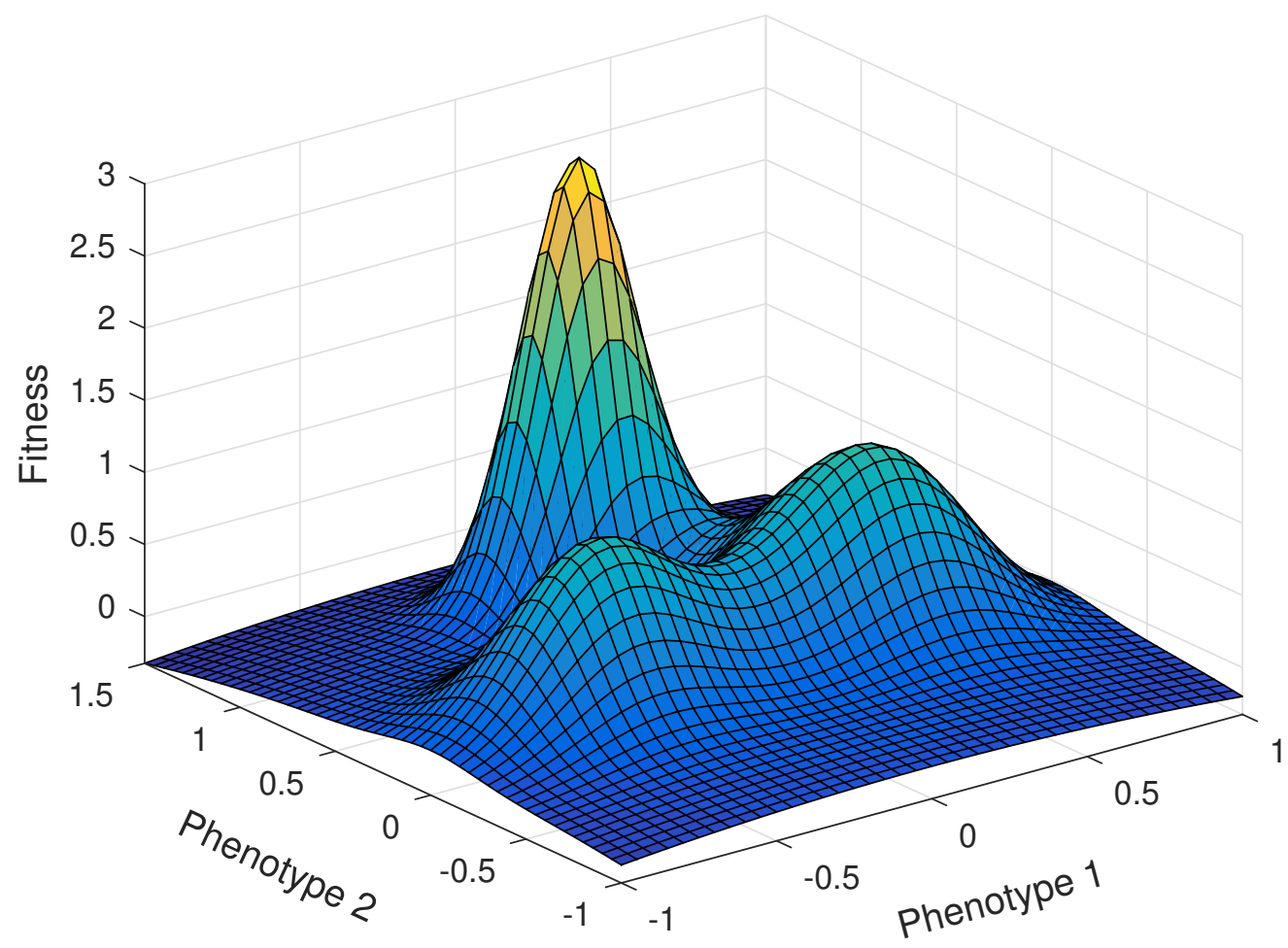

Figure 1. Fitness function used in the Fisher-Eigen equation

We modeled this process with a partial differential equation simulation of a discretized representation of the phenotype space around the peaks of the fitness function. We discretized the space and simulation time steps sufficiently to accu- 
rately capture the dynamic and steady state behavior of the Fisher-Eigen equation. We also tested the sensitivity of the responses to discretization granularity. A typical choice for the setup was a simulation area of $[-1.5,1.5] \times[-1.5,1.5]$ and the time interval $[0,25]$ using $\Delta x_{1}=\Delta x_{2}=0.04$ and $\Delta t=0.001$. We modeled $p$ by

$$
\frac{p(\mathbf{x}, t+\Delta t)-p(\mathbf{x}, t)}{\Delta t}=(\bar{U}(t)-U(\mathbf{x})) p(\mathbf{x}, t)+D \nabla_{h}^{2} p(\mathbf{x}, t),
$$

where

$$
\bar{U}(t)=\sum U(\mathbf{x}) p(\mathbf{x}, t) \Delta x_{1} \Delta x_{2}
$$

is the average energy and

$$
\begin{aligned}
\nabla_{h}^{2} p\left(\left(x_{1}, x_{2}\right), t\right) & =\frac{p\left(\left(x_{1}+\Delta x_{1}, x_{2}\right), t\right)+p\left(\left(x_{1}-\Delta x_{1}, x_{2}\right), t\right)-2 p\left(\left(x_{1}, x_{2}\right), t\right)}{\left(\Delta x_{1}\right)^{2}} \\
& +\frac{p\left(\left(x_{1}, x_{2}+\Delta x_{2}\right), t\right)+p\left(\left(x_{1}, x_{2}-\Delta x_{2}\right), t\right)-2 p\left(\left(x_{1}, x_{2}\right), t\right)}{\left(\Delta x_{2}\right)^{2}}
\end{aligned}
$$

is a finite difference operator representing the diffusion term. We added a Dirichlet boundary condition $p(\mathbf{x}, t)=0$ whenever $\mathbf{x}$ lies on the boundary of the simulation area. We also tried Neumann boundary conditions of $\nabla p(\mathbf{x}, t)=0$ and obtained similar results. We used the RK4 method to time step based on this equation.

We chose time steps sufficiently small such that

$$
p(\mathbf{x}, t)>0, \forall \mathbf{x} \Rightarrow p(\mathbf{x}, t+\Delta t)>0, \forall \mathbf{x}
$$

Equation 2.

so that $p$ would remain positive in all cases. Assuming $p$ is positive everywhere at time $t$, then $D \nabla_{h}^{2} p(\mathbf{x}, t)>\frac{-4 D p(\mathbf{x}, t)}{\left(\Delta x_{1}\right)^{2}}$, since the tiles are square so $\Delta x_{1}=\Delta x_{2}$. Furthermore, we see that $(\bar{U}(t)-U(\mathbf{x})) p(\mathbf{x}, t)>-3.5 p(\mathbf{x}, t)$ by finding the extrema of $U$ with numerical methods. Substituting $\frac{p(\mathbf{x}, t+\Delta t)-p(\mathbf{x}, t)}{\Delta t}>\left(-3.5-\frac{4 D}{\left(\Delta x_{1}\right)^{2}}\right) p(\mathbf{x}, t)$ into Equation 2 gives the $\Delta t$ constraint for the full PDE:

$$
\Delta t \leq\left(3.5+\frac{4 D}{\left(\Delta x_{1}\right)^{2}}\right)^{-1}
$$

In particular, choosing $\Delta t=0.001$ and $\Delta x_{1}=\Delta x_{2}=0.04$ works as long as $D \leq 0.39$. After each time step, we divided $p$ by its integral $\sum p(\mathbf{x}, t) \Delta x_{1} \Delta x_{2}$ so that $p$ would remain a probability distribution, to account for any loss of population, for example due to numerical precision.

To analyze the dynamics of this system, we split the space into 3 regions corresponding to the nodes of the network function that will model the system as an ODE. We chose basic linear region partitions based on the centers of the three fitness peaks in this model. Future work could study whether other partitioning schemes add more accuracy such as using saddle lines, equipotential-like isolines, or other model-based partition lines. The behavior of each region in the PDE will be lumped into an ODE equation for the network model:

$$
\begin{aligned}
& \Omega_{1}=\left\{\left(x_{1}, x_{2}\right) \in[-1.5,1.5] \times[-1.5,1.5]: x_{2}>\left|\frac{\sqrt{3}}{3} x_{1}\right|+\frac{\sqrt{3}}{6}\right\} \\
& \Omega_{2}=(0,1.5] \times[-1.5,1.5] \backslash \Omega_{1} \\
& \Omega_{3}=[-1.5,0] \times[-1.5,1.5] \backslash \Omega_{1} .
\end{aligned}
$$


These regions are depicted in Figure 2.

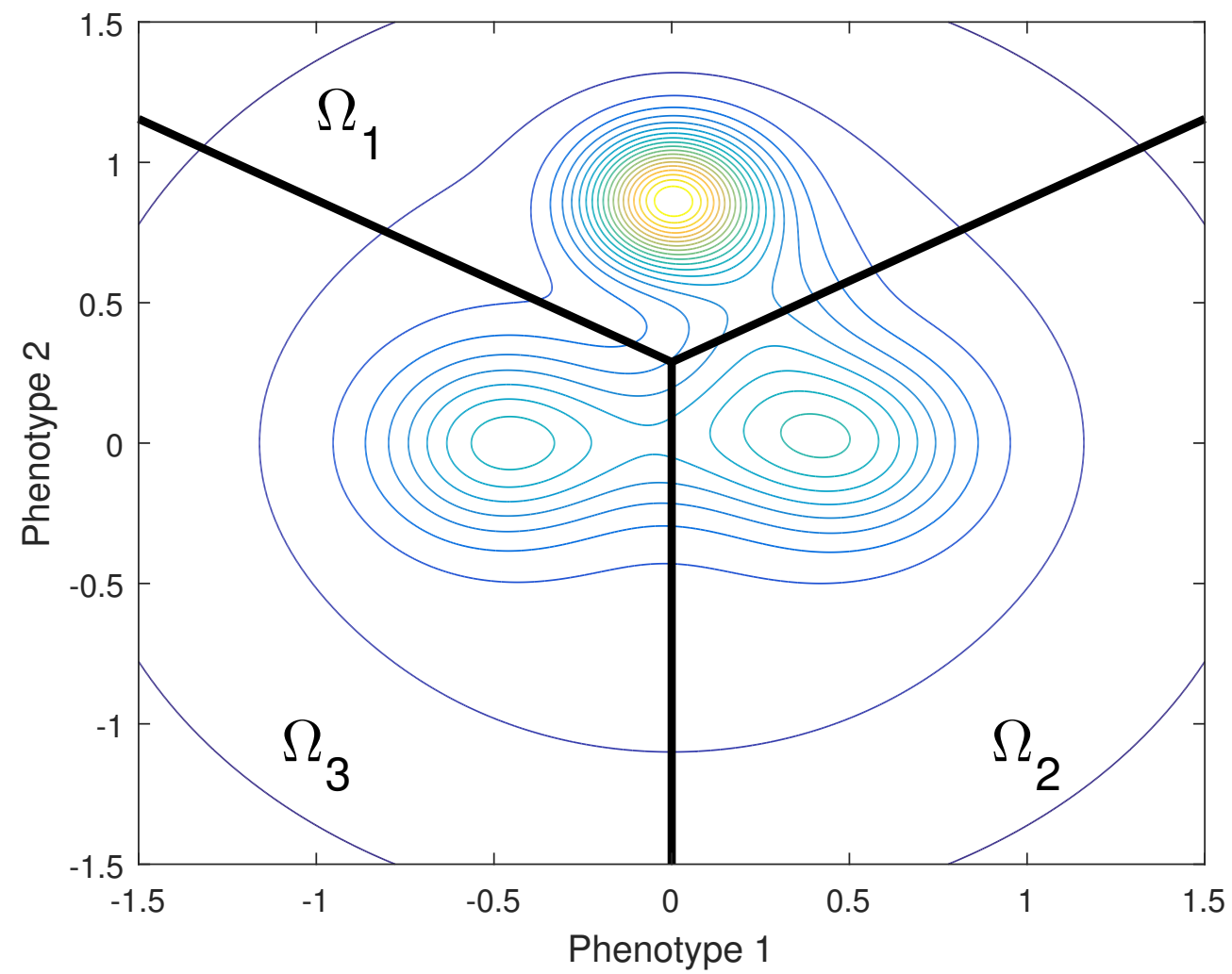

Figure 2. Partitioning the phenotype space into regions corresponding to network nodes. Contours show the fitness function peaks.

Let $I_{i}=\int_{\Omega_{i}} p(\mathbf{x}, t) d \mathbf{x}$, which represents the total population in region $\Omega_{i}$. From the equation

$$
\frac{\partial p(\mathbf{x}, t)}{\partial t}=(\bar{U}(t)-U(\mathbf{x})) p(\mathbf{x}, t)+D \nabla^{2} p(\mathbf{x}, t)
$$

we integrate both sides over the region $\Omega_{i}$ to obtain

$$
\int_{\Omega_{i}} \frac{\partial p(\mathbf{x}, t)}{\partial t} d \mathbf{x}=\bar{U}(t) \int_{\Omega_{i}} p(\mathbf{x}, t) d \mathbf{x}-\int_{\Omega_{i}} U(\mathbf{x}) p(\mathbf{x}, t) d \mathbf{x}+D \int_{\partial \Omega_{i}}(\nabla p(\mathbf{x}, t)) \cdot \vec{n} d s,
$$

Equation 3.

where $\partial \Omega_{i}$ is the boundary of $\Omega_{i}$ and $\vec{n}$ is the outward-pointing unit normal to $\partial \Omega_{i}$. The last term follows by the $\mathrm{Di}$ vergence Theorem. Differentiating under the integral sign, we see that the left hand side is $\frac{d I_{i}}{d t}$. The last term on the right hand side is the flux through $\Omega_{i}$, which because of the boundary condition is the flux through the two line segments surrounding $\Omega_{i}$. If we let $J_{i j}$ be the flux from $\Omega_{j}$ to $\Omega_{i}$ through the segment bordering $\Omega_{i}$ and $\Omega_{j}$, we have $J_{i j}=-J_{j i}$ and $J_{i j}+J_{i k}=D \int_{\partial \Omega_{i}}(\nabla p(\mathbf{x}, t)) \cdot \vec{n} d s$ when $i, j, k$ are all distinct. Then Equation 3 becomes

$$
\frac{d I_{i}}{d t}=\bar{U}(t) \int_{\Omega_{i}} p(\mathbf{x}, t) d \mathbf{x}-\int_{\Omega_{i}} U(\mathbf{x}) p(\mathbf{x}, t) d \mathbf{x}+\sum_{j \neq i} J_{i j} .
$$

Equation 4. 
We approximated the flux across each boundary segment by discretizing the segment into $N$ equally spaced points $\left\{\mathbf{x}_{1}, \mathbf{x}_{2}, \cdots, \mathbf{x}_{N}\right\}$ and taking the summation $D \sum_{i=1}^{N} \nabla p\left(\mathbf{x}_{i}, t\right) \cdot \vec{n} d s$, where $d s=\frac{L}{N}, L$ is the length of the segment, and $\vec{n}$ is the unit normal vector to the segment bordering $\Omega_{i}$ and $\Omega_{j}$ going from $\Omega_{i}$ to $\Omega_{j}$. We found that $N=15$ was sufficient to obtain an accurate approximation by testing the sensitivity of the result to line segment length, as we did with area and time discretization.

Let $U_{i}(t)=\frac{\int_{\Omega_{i}} U(\mathbf{x}) p(\mathbf{x}, t) d \mathbf{x}}{\int_{\Omega_{i}} p(\mathbf{x}, t) d \mathbf{x}}$. Because in each region we expect the population to be relatively stable around the peak, we assume that $U_{i}(t)$ is approximately constant. Thus, we can approximate $U_{i}(t)$ by its value at equilibrium, which is denoted $U_{i}^{\mathrm{eq}}$. At equilibrium, we have $\frac{d I_{1}}{d t}=\frac{d I_{2}}{d t}=\frac{d I_{3}}{d t}=0$. Note that Equation 4 is unchanged if we add a constant to $U(\mathbf{x})$. Hence, we may assume that $\bar{U}(t)=0$ at equilibrium. Then Equation 4 becomes, at equilibrium,

$$
0=-U_{i}^{\mathrm{eq}} I_{i}^{\mathrm{eq}}+J_{i j}^{\mathrm{eq}}+J_{i k}^{\mathrm{eq}} \text {. }
$$

Equation 5.

We can rearrange this equation and use the fact that $J_{i j}=-J_{j i}$ to solve for $U_{i}^{\mathrm{eq}}$ in terms of three of the fluxes and the $I_{i} \mathrm{~s}$.

$$
\begin{aligned}
U_{1}^{\mathrm{eq}} & =\frac{-J_{21}^{\mathrm{eq}}-J_{31}^{\mathrm{eq}}}{I_{1}^{\mathrm{eq}}} \\
U_{2}^{\mathrm{eq}} & =\frac{J_{21}^{\mathrm{eq}}-J_{32}^{\mathrm{eq}}}{I_{2}^{\mathrm{eq}}} \\
U_{3}^{\mathrm{eq}} & =\frac{J_{31}^{\mathrm{eq}}+J_{32}^{\mathrm{eq}}}{I_{3}^{\mathrm{eq}}} .
\end{aligned}
$$

Equation 6.

Equation 7.

Equation 8.

We approximate the flux $J_{i j}$ as being linear in $I_{i}$ and $I_{j}$. In the case of a Smoluchowski process, such an assumption is reasonable because particles move essentially independently of the global movement of all the particles, so the rate of movement between peaks depends only on the number of organisms at each peak. In contrast, for the Fisher-Eigen process the chances of an organism surviving a crossing depends on the organism's fitness relative to the entire population, so it is not immediately clear that the flux can be linear in each population. We set up a linear flux model for Fisher-Eigen and show the process with details explained in the following paragraphs.

We wish to model $J_{i j}$ as $k_{j i} I_{j}-k_{i j} I_{i}$, where $k_{i j}$ is the transition rate from $i$ to $j$. We assume that the transition rates are independent of time. Assuming such an approximation holds, from the equilibrium data we obtain 3 equations for the 6 unknown transition rates. In theory any set of transition rates that satisfies these equations will give an equilibrium solution for the ODE model that matches that of the PDE simulation. However, to ensure the ODE model follows the dynamic behavior of the PDE simulation, the transition rates should be chosen so that the flux approximations are accurate over time. Therefore, we used the least squares method to find the transition rates by comparing the actual flux over time extracted from the PDE simulation with the linear model of flux over time. We ran a transient simulation with a starting population far from equilibrium, and ran the simulation until the population and flux settled into equilibrium values. We split the time interval into equally spaced points $t_{1}, t_{2}, \cdots, t_{M}$, typically using 300 to 600 sample points. 
Consider the following matrices:

$$
X_{1}=\left(\begin{array}{cc}
I_{2}\left(t_{1}\right) & I_{3}\left(t_{1}\right) \\
I_{2}\left(t_{2}\right) & I_{3}\left(t_{2}\right) \\
\vdots & \vdots \\
I_{2}\left(t_{M}\right) & I_{3}\left(t_{M}\right)
\end{array}\right), \quad Y_{1}=\left(\begin{array}{c}
J_{32}\left(t_{1}\right) \\
J_{32}\left(t_{2}\right) \\
\vdots \\
J_{32}\left(t_{M}\right)
\end{array}\right)
$$

We found a vector $\beta_{1}=\left(k_{23},-k_{32}\right)^{T}$ that minimized $\left|Y_{1}-X_{1} \beta_{1}\right|^{2}$. Similarly, we found vectors $\beta_{2}=\left(k_{13},-k_{31}\right)^{T}$ and $\beta_{3}=\left(k_{12},-k_{21}\right)^{T}$ for the corresponding matrices for $J_{31}$ and $J_{21}$.

We chose initial conditions that put $98 \%$ of the population in region 3, in a narrow Gaussian centered on the fitness peak in that region. Finding such a starting population $p(\mathbf{x}, 0)=p_{0}(\mathbf{x})$ required estimating $\int_{\Omega_{3}} p_{0}(\mathbf{x}) d \mathbf{x}$. To do this, we used a quadrature scheme analogous to that of the entire domain, choosing $\Delta x_{1}=\Delta x_{2}=0.04$. We took the summation $\sum_{\mathbf{x} \in \Omega_{3}} p_{0}(\mathbf{x}) \Delta x_{1} \Delta x_{2}$ over all squares contained within $\Omega_{3}$. We then varied the parameters of the Gaussian $p_{0}$ so that this summation would be approximately 0.98 .

As the simulation progressed to equilibrium, population flowed to regions 1 and 2 with the final value in region 3 being the smallest. The final values in all three regions depend on the fitness function and the diffusion constant $D$. At the beginning of the simulation, there is a short lag time while the initially applied population starts to flow resulting in rapid flux changes briefly. Thus, using $t_{1}=0$ causes error in computing the $k_{i j}$ 's. We typically started flux samples for the least squares fit calculations at approximately time $t_{1}=1$ out of a total simulation time of 25 .

With the transition rates, we obtain a system of ODEs that models $I_{1}, I_{2}$, and $I_{3}$.

$$
\begin{aligned}
\frac{d I_{1}}{d t} & =\left(\sum_{i=1}^{3} U_{i}^{\mathrm{eq}} I_{i}-U_{1}^{\mathrm{eq}}\right) I_{1}-k_{12} I_{1}+k_{21} I_{2}-k_{13} I_{1}+k_{31} I_{3} \\
\frac{d I_{2}}{d t} & =\left(\sum_{i=1}^{3} U_{i}^{\mathrm{eq}} I_{i}-U_{2}^{\mathrm{eq}}\right) I_{2}-k_{23} I_{2}+k_{32} I_{3}-k_{21} I_{2}+k_{12} I_{1} \\
\frac{d I_{3}}{d t} & =\left(\sum_{i=1}^{3} U_{i}^{\mathrm{eq}} I_{i}-U_{3}^{\mathrm{eq}}\right) I_{3}-k_{31} I_{3}+k_{13} I_{1}-k_{32} I_{3}+k_{23} I_{2} .
\end{aligned}
$$

We then simulated this system of ODEs using MATLAB's built in ode45 function. We used the same time interval as in the PDE simulation, typically $[0,25]$. The initial conditions on $I_{i}$ were the same as the initial conditions of the PDE simulation, with $98 \%$ of the population starting in region 3.

\section{RESULTS}

We ran the simulation outlined in Methods and Procedures with $D$ varying between 0.01 and 0.2 . Figure 3 shows the equilibrium population distribution computed by the PDE simulation. Figure 4 shows the initial population distribution with the sharp peak in region 3. To check the accuracy of the linear model of flux, we plotted it against the flux that was computed as previously described during the transient response of the PDE simulation. The plots of $J_{32}, J_{31}$, and $J_{21}$ for both $D=0.01$ and $D=0.09$ are shown in Figure 5 . 


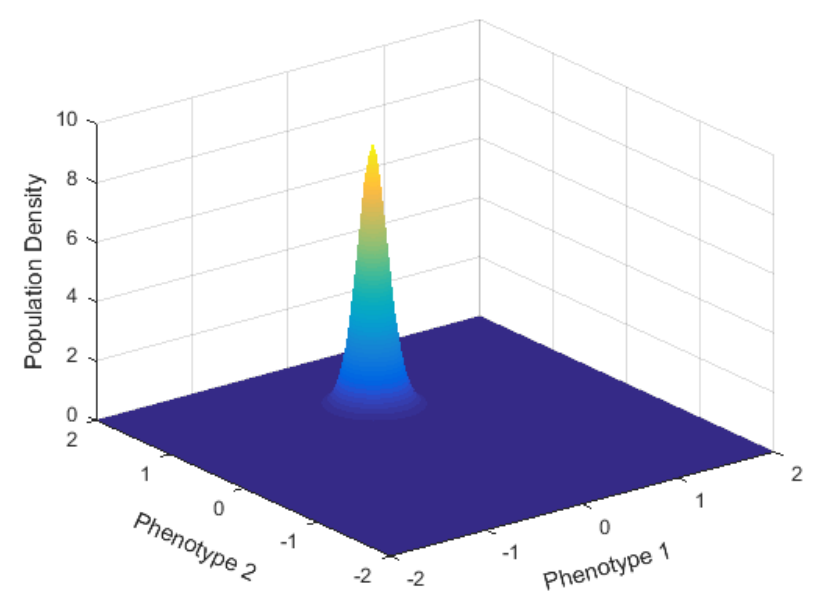

(a) $D=0.01$

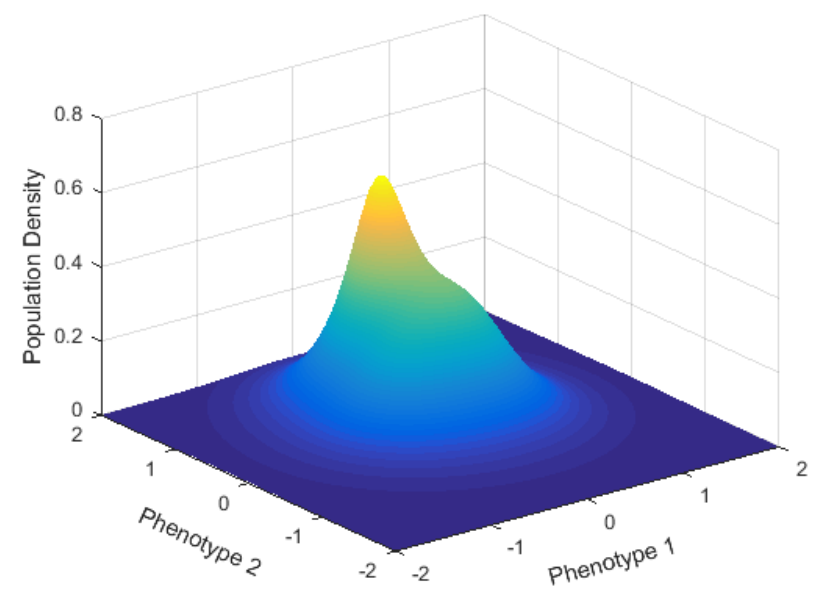

(c) $D=0.09$

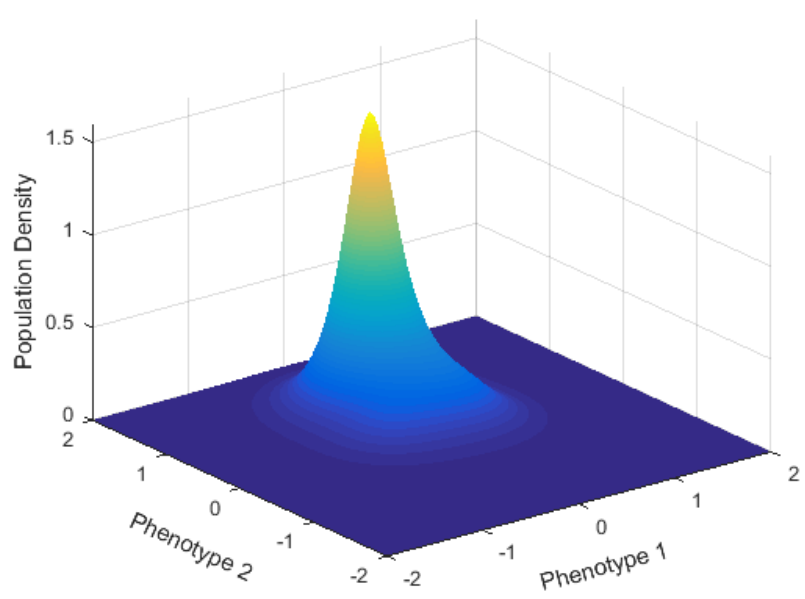

(b) $D=0.05$

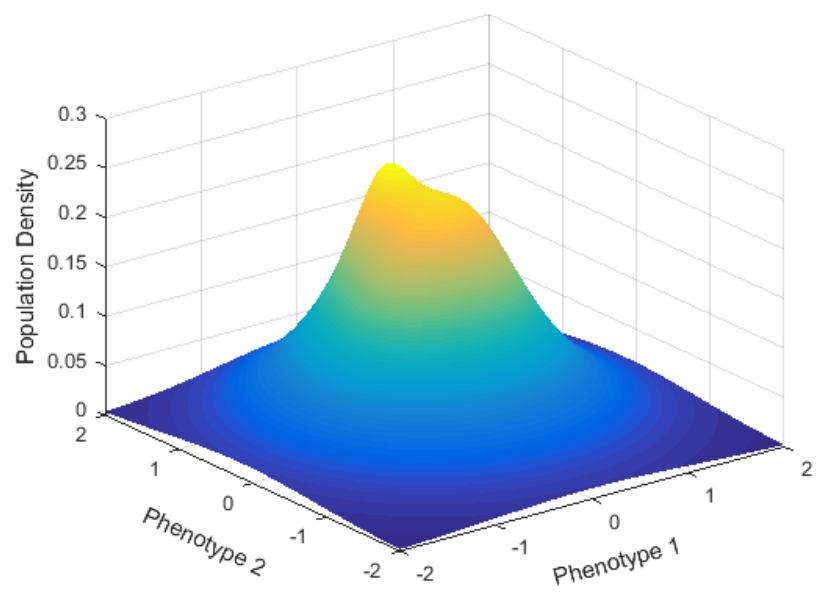

(d) $D=0.20$

Figure 3. Equilibrium population distribution computed by PDE simulations for various $\mathrm{D}$ values 


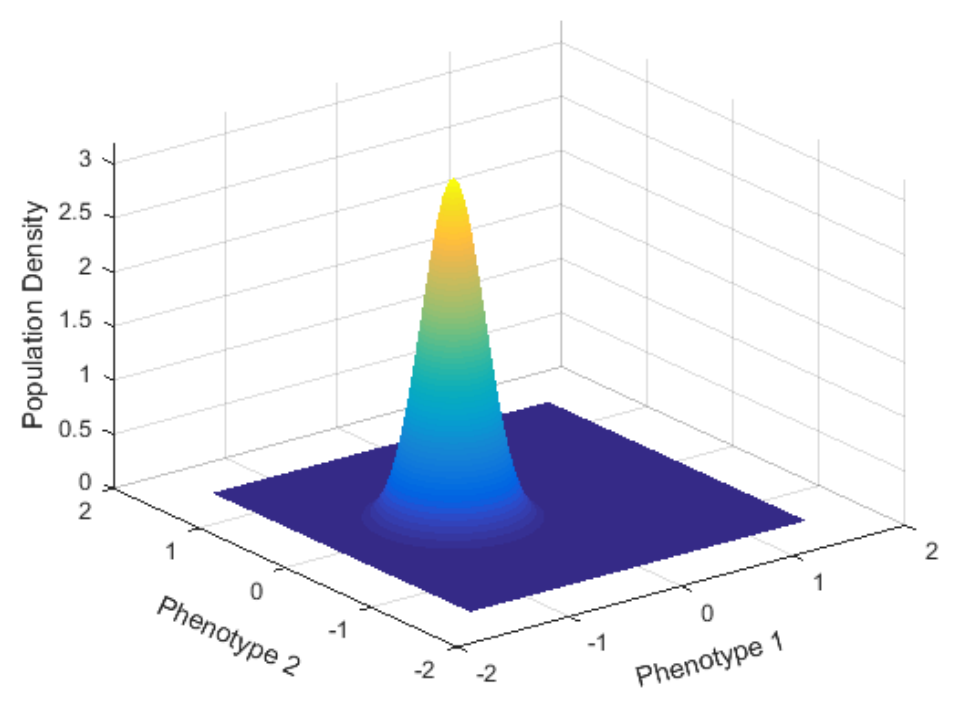

Figure 4. Starting population for transient simulations has $98 \%$ of the population in region 3

Figure 5 provides numerical evidence that for both large and small $D$, the linear models are an accurate approximation of the flux terms. The linear models all approximate the long-term behavior of the actual fluxes, and in general the flux and linear model are similar qualitatively. The largest error in these models appears for small $t$, which indicates that there still may be a loss of accuracy due to a lag time at the start of the simulation.

Once we obtained the transition rates from these models, we used Equation 6, Equation 7, and Equation 8, along with the equilibrium data from the PDE simulation, to find the remaining coefficients $U_{i}^{\text {eq }}$. We then simulated $I_{1}, I_{2}$, and $I_{3}$ by a system of ODEs, as described in Section 3. Figure 6 shows the values of $I_{i}$ over time as described by the PDE simulation and by the ODE model for $D=0.09, D=0.05$ and $D=0.01$, respectively. Table 1 shows the values of $k_{i j}$ and $U_{i}^{\text {eq }}$ used for each of the simulations shown in Figure 6.

The ODE model has the desired property of reaching the same equilibrium state as the actual PDE simulation. Qualitatively the graphs show similar dynamics. However, the time the ODE model takes to reach equilibrium is sometimes slightly less than the time the PDE model takes, particularly for small $D$.

One possible cause of the increased rate for the ODE model is that there is no lag to move around a peak. In the PDE model, once an organism has entered one of the three regions it takes time for that organism to move within that region to reach a point where it can move to another region. In contrast, in the ODE model, once an organism moves to a region it can instantly move to a different region at the next time step. The lag is more pronounced with slower diffusion (lower $D$ values). Also for these trial simulations to validate the mathematical models, the population starts at an artificially narrow peak in the interior of one region, so minimal flux is present until some population reaches an edge, during which the PDE simulations show no population changes initially. For a more natural initial population smoothly distributed through the phenotype space, some flux will be present at the region boundaries which will minimize the PDE response lag times and allow the PDE and ODE responses to match more closely.

Based on our trials, the ODE model will reach an equilibrium state determined only by the transition rates $k_{i j}$ and average fitness $U_{i}$. That is, one can change the initial distribution of the population without changing the equilibrium solution. For example, instead of having all the population start at the lowest fitness peak, one could have them all start at the middle peak or the highest peak. Figure 7 shows the results from running the $D=0.09$ case with those three initial conditions, using parameters $k_{i j}$ and $U_{i}^{\mathrm{eq}}$ determined by the $D=0.09$ case analyzed for Figures 5 and 6 . 


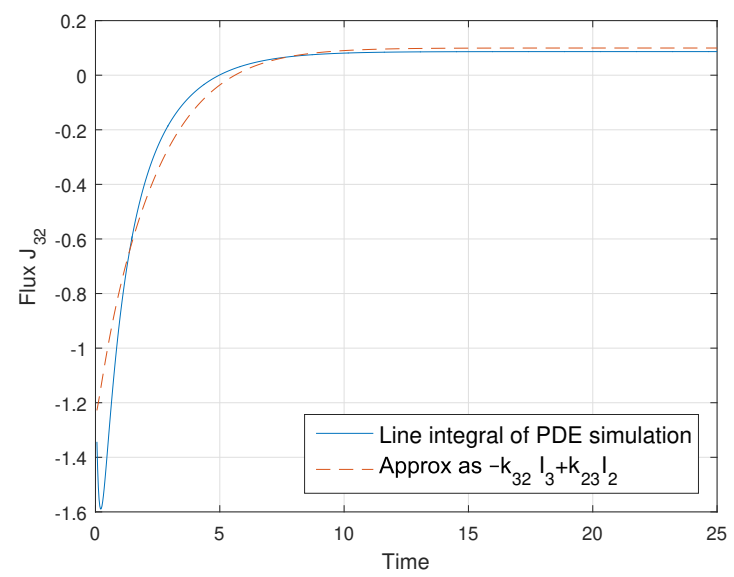

(a) $J_{32} ; D=0.09$

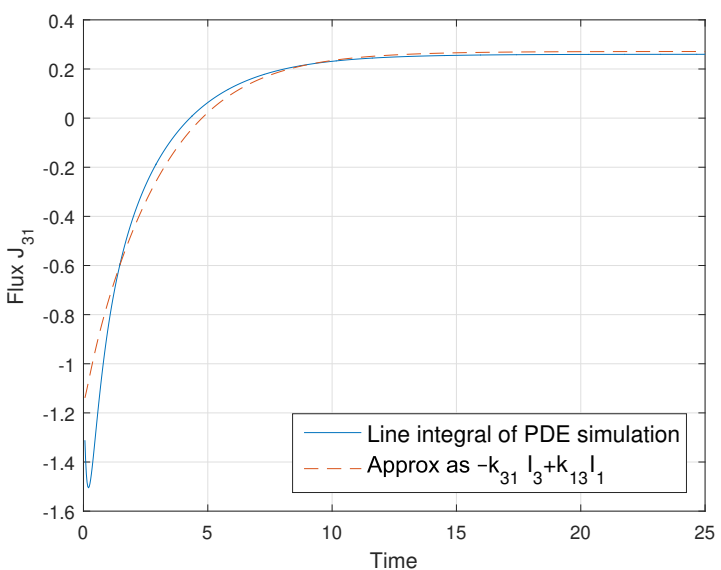

(c) $J_{31} ; D=0.09$

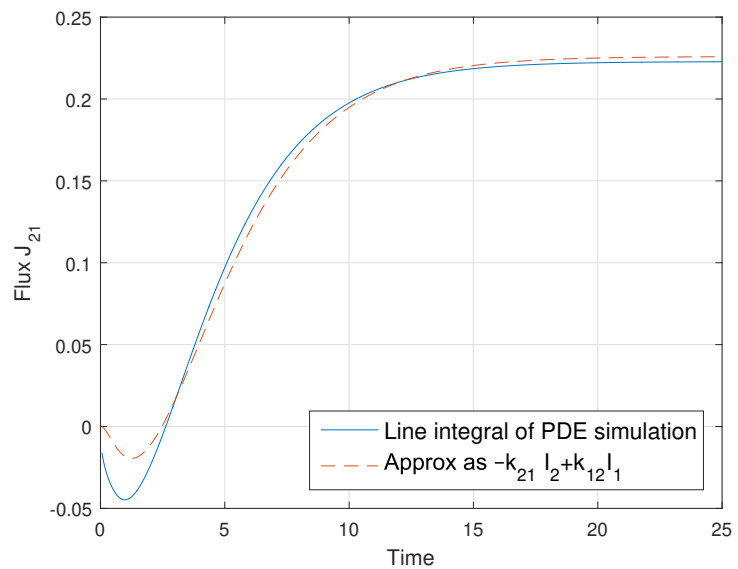

(e) $J_{21} ; D=0.09$

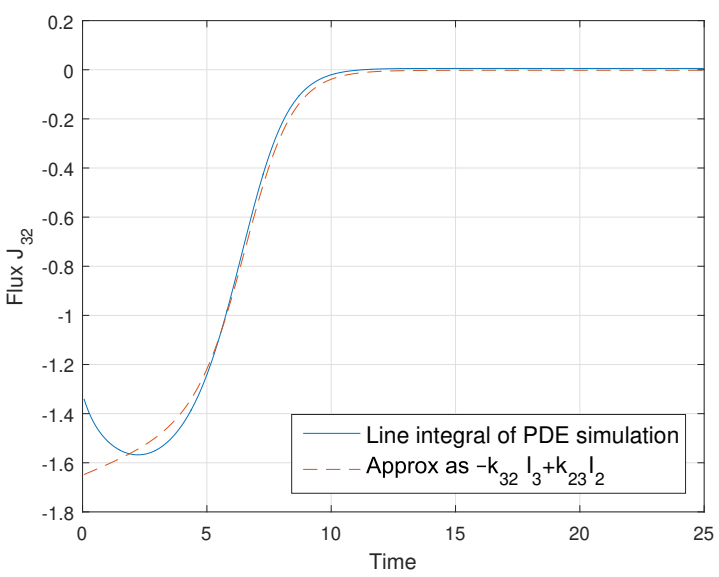

(b) $J_{32} ; D=0.01$

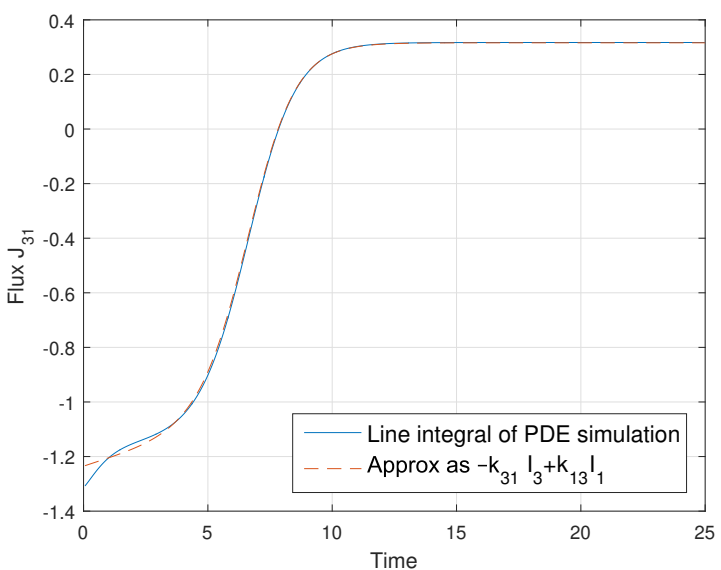

(d) $J_{31} ; D=0.01$

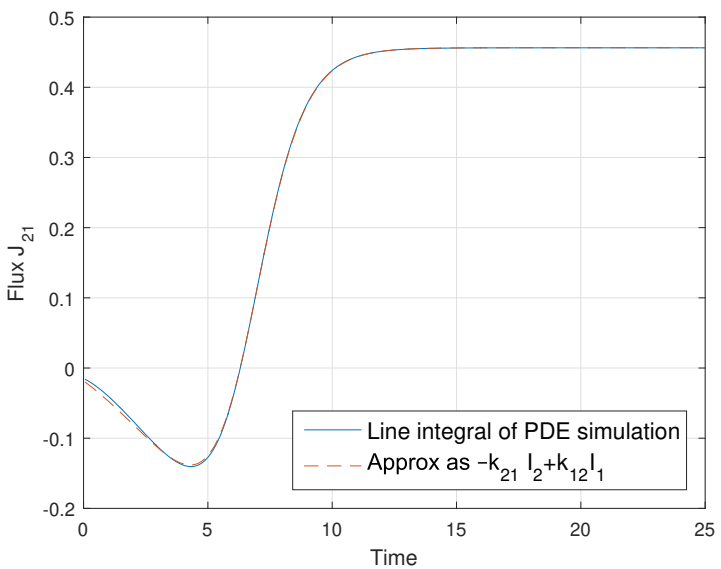

(f) $J_{21} ; D=0.01$

Figure 5. Comparison of the linear model of flux with the actual flux 


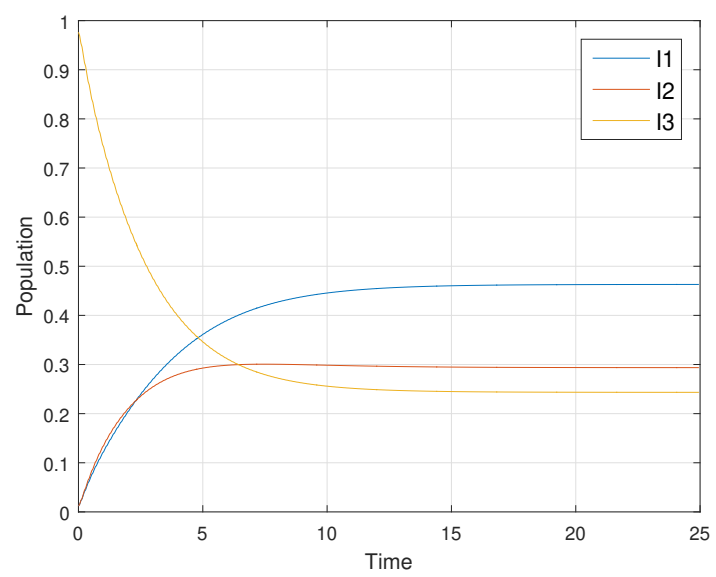

(a) PDE Simulation; $D=0.09$

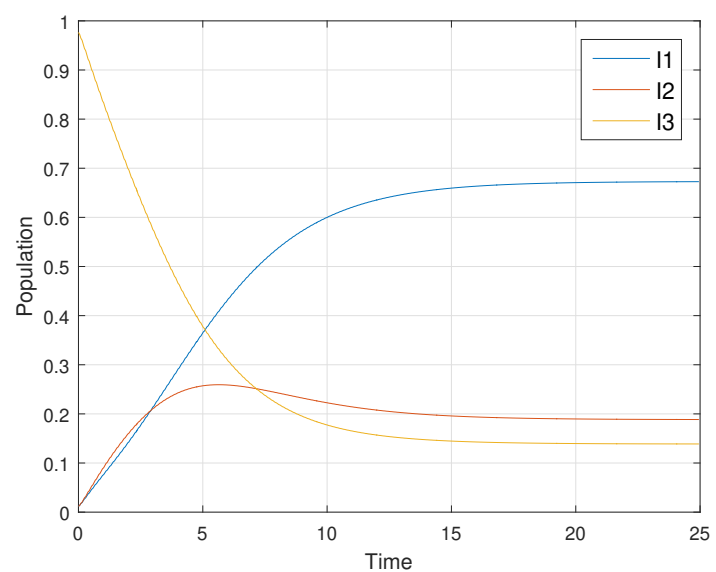

(c) PDE Simulation; $D=0.05$

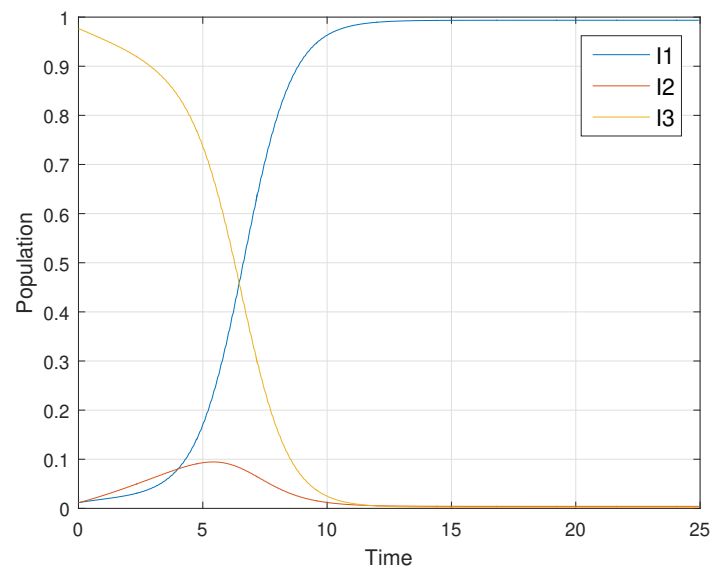

(e) PDE Simulation; $D=0.01$

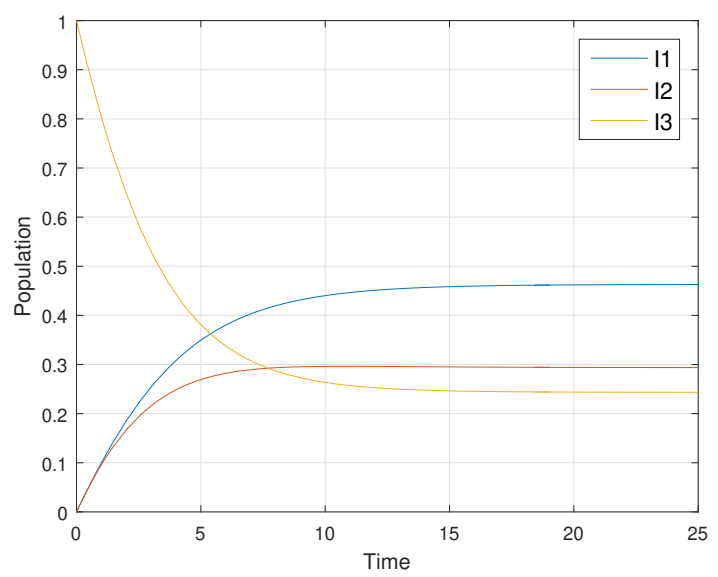

(b) ODE Model; $D=0.09$

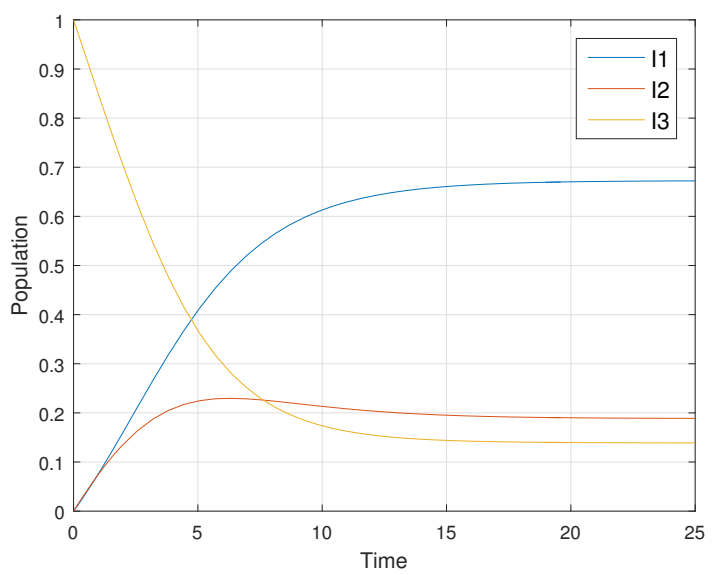

(d) ODE Model; $D=0.05$

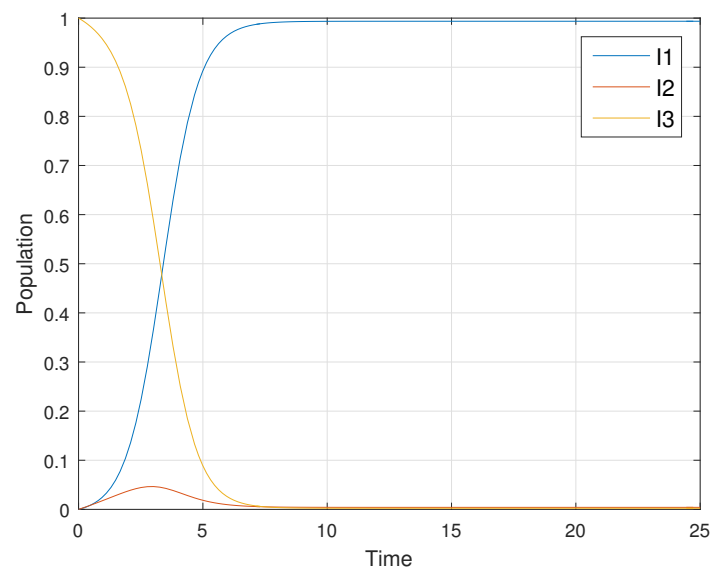

(f) ODE Model; $D=0.01$

Figure 6. Comparison of the PDE simulation with the ODE model for various D values. Initial condition: $98 \%$ of population starts in a narrow peak in region 3. 


\begin{tabular}{|c|c|c|c|c|c|c|c|c|c|}
\hline$D$ & $k_{12}$ & $k_{13}$ & $k_{21}$ & $k_{23}$ & $k_{31}$ & $k_{32}$ & $U_{1}^{\text {eq }}$ & $U_{2}^{\text {eq }}$ & $U_{3}^{\text {eq }}$ \\
\hline 0.09 & 0.1221 & 0.1086 & 0.1233 & 0.1256 & 0.1063 & 0.1148 & -0.0950 & 0.0403 & 0.1321 \\
\hline 0.05 & 0.0743 & 0.0650 & 0.0819 & 0.0934 & 0.0698 & 0.0808 & -0.1020 & 0.1493 & 0.2915 \\
\hline 0.01 & 0.0047 & 0.0032 & 0.0218 & 0.0043 & 0.0127 & 0.0169 & -0.0078 & 1.1193 & 1.3732 \\
\hline
\end{tabular}

Table 1. Coefficients of the ODE Model for the simulations shown in Figure 6.

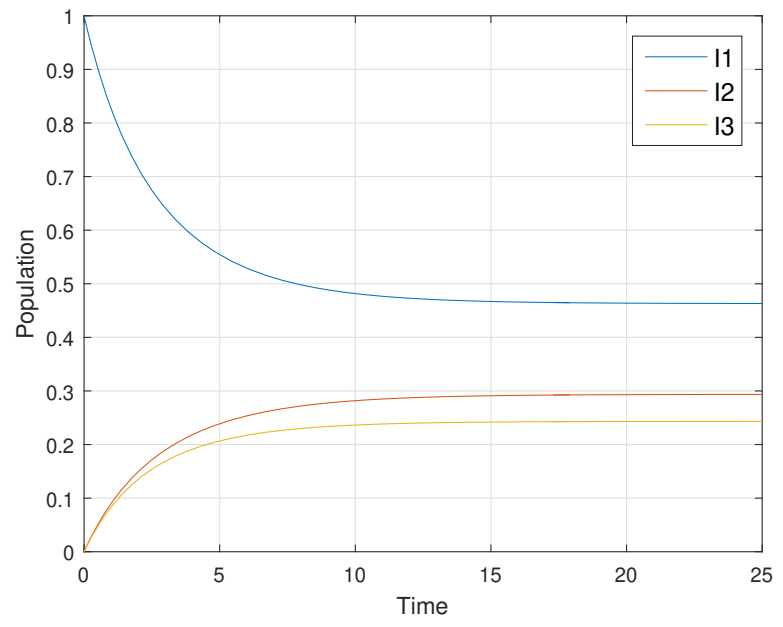

(a) Start at high peak

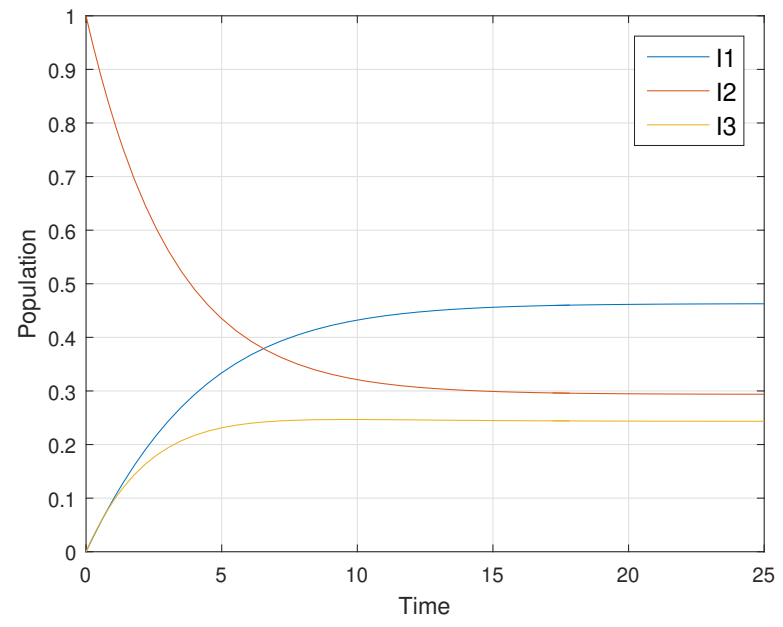

(b) Start at middle peak

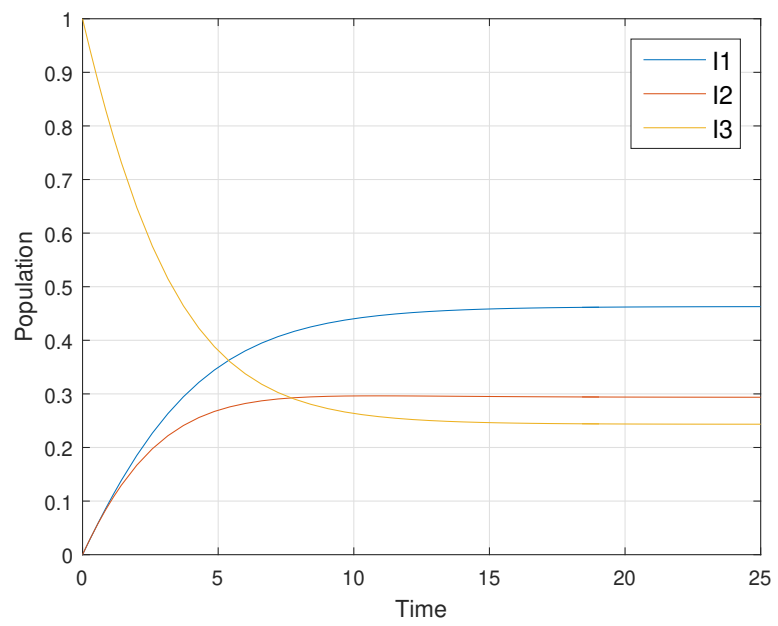

(c) Start at low peak

Figure 7. Results of the ODE model under the same parameters and three different initial conditions; $D=0.09$

As an example of a practical application of the ODE model, highlighting the precision of the equilibrium state as well as the well-matched transient response, Figure 8 shows the population evolution after a sudden (step) change in the environment affecting the fitness function. Returning to the example of a colony of bacteria, if an antibiotic were introduced that targeted bacteria whose phenotypes were similar to those of $\Omega_{2}$, the fitness function for their environment would undergo a sudden change. In the example run in Figure 8, the fitness peak in region 2 drops by a factor of 4 due to the antibiotic. For the ODE model, the values of $U_{i}^{\text {eq }}$ are computed as before (using the equilibrium data with Equation 6, Equation 7, and Equation 8) so as to match the modified fitness function, but the transition rates for the 
original fitness function are used. Both the PDE and ODE models were run in this new setup, where the initial conditions were the equilibrium solutions of the original setup. The PDE and ODE transient responses are very similar, showing the decline in the region 2 population over time and relative increase in region 1 and 3 populations (which are not targeted by the antibiotic). In this case, the ODE model with 3 nodes generates nearly the same results as the PDE simulation with over hundreds of grid points and thousands of time steps. This clearly demonstrates the potential of a network model for the Fisher-Eigen equation, which could be scaled up to much more complex fitness functions and phenotype spaces.

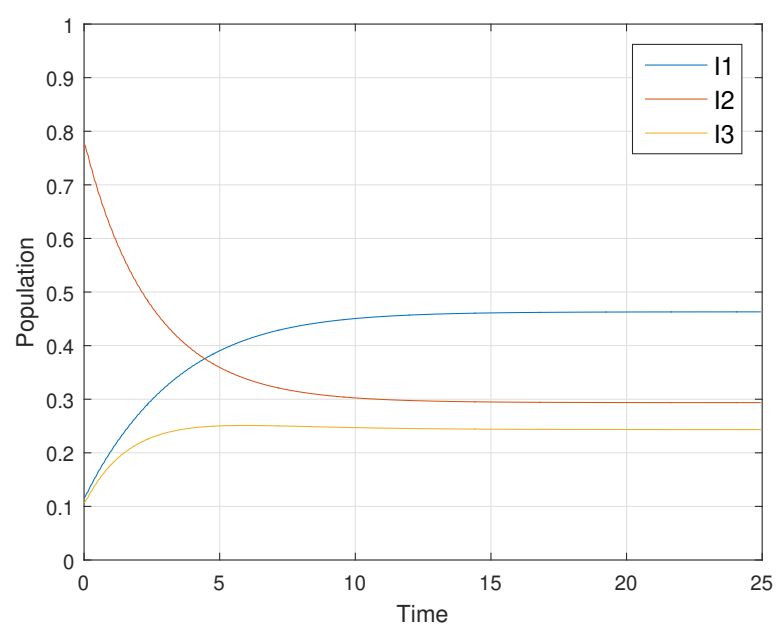

(a) PDE Simulation

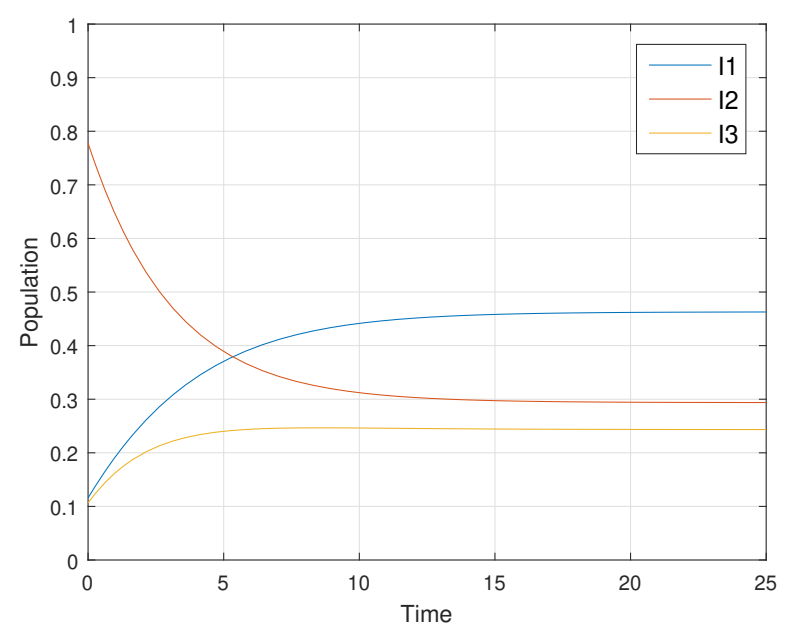

(b) ODE Model

Figure 8. Comparison of the PDE and ODE response to a sudden change in fitness environment; $D=0.09$

\section{CONCLUSIONS}

This paper detailed a simulation of the Fisher-Eigen process for a 2-dimensional fitness landscape, and it explained the use of this simulation in constructing a model of the Fisher-Eigen process as a system of ODEs over a network. The results indicated that the flux between two nodes in the network could be approximated as a linear combination of the integrals of the probability density over each region. The results also showed that this ODE model reached wellmatched transient and precise equilibrium solutions. The motivation for constructing such a model was to simplify the modeling of high-dimensional fitness landscapes and model dynamic behavior with data taken from equilibrium conditions. This prototype can be replicated by others, and the savings in computational complexity could enable the inclusion of far more phenotypes and more complicated fitness functions than can be used in current Fisher-Eigen models. These new models could illuminate behavior for populations previously too complex to predict. The models could be used to combat the growing public health menace of antimicrobial resistant pathogens, for example, as well as many other applications.

To implement this model in practice, one would need to find the transition rates $k_{i j}$ without relying on the flux data taken over time. One such method would be to derive analytic expressions for these transition rates. The method for doing so would be similar to that of previous work. ${ }^{5}$ First, use spectral analysis to determine an equation for the probability density $p(\mathbf{x}, t)$ in terms of eigenfunctions. Then, truncate the eigenfunction expansion to obtain an approximate solution for $p(\mathbf{x}, t)$. After solving the master equation in terms of the transition rates, compare the expression it gives for $I_{i}(t)$ with the one given by integrating the truncated eigenfunction expansion over $\Omega_{i}$. From this equation derive approximate expressions for the transition rates in terms of the first few eigenvalues. 
Another direction of future work could be to prove some of these results analytically. One could attempt to prove that the initial conditions do not affect the equilibrium state of the ODE model, and further that the ODE model is asymptotically stable, which it appears to be in practice. A more difficult task would be to determine for which cases the flux is approximated well by linear models. Based on our trials, we would expect the approximation to be better for smaller $D$.

We would also like to study the dynamics of the ODE model where it differs at a fine grain level from those of the PDE model. The network model is a lumped model of the system, which assumes state variables of a node such as population density apply everywhere in the node. Slow diffusion with low $D$ values results in larger differences in population density across a node, making the accuracy of a single lumped model more problematic compared to the PDE model. With this in mind, one possible modification to the ODE model would be to generate additional subnodes to model diffusion within a top-level model based on $D$ and the size of the region. Also, adding transition nodes between each region may capture additional diffusion effects. Another aspect of improving the ODE model would be studying the initial conditions such that the ODE model could match the PDE more closely at time $t_{1}=0$.

Another area of focus for future work would be other partitioning schemes and criteria for mapping a phenotype space onto a network of nodes based on the biological data. For larger and more complex systems, these methods would require algorithms and automation. We would also like to extend this work to fitness functions other than the one chosen. It would be interesting to consider fitness functions which produce much more complicated networks, in which organisms have many viable paths to reach peaks. Also, we would like to consider fitness functions in higher dimensions. A 2-dimensional fitness function is a reasonable starting place, but it does not capture the complexity that would be involved in modeling in higher dimensions.

\section{REFERENCES}

1. Feistel, R. and Ebeling, W. (1998) Evolution of Complex Systems: Selforganization, Entropy and Development, Springer, New York.

2. Eigen, M. (1971) Selforganization of matter and the evolution of biological macromolecules, Naturwissenschaften, volume 58, number 10, 465-523. https://doi.org/10.1007/BF00623322

3. Fisher, R. A. (1930) The genetical theory of natural selection, Oxford University Press, Oxford. bttps://doi.org/10.5962/bbl.title.27468

4. Ebeling, W. and Feistel, R. (2018) Studies on Manfred Eigen's model for the self-organization of information processings, European Biophysics Journal, volume 47, number 4, 395-401. https://doi.org/10.1007/s00249-018-1287-1

5. Dunkel, J. and Ebeling, W. and Schimansky-Geier, L. and Hänggi, P. (2003) Kramers problem in evolutionary strategies, Physical Review E, volume 67, number 6, 061118 1-11. https://doi.org/10.1103/physreve.67.061118

6. Asselmeyer, T. and Ebeling, W. and Rosé, H. (1997) Evolutionary strategies of optimization, Physical Review E, volume 56, number 1, 1171-1180. https://doi.org/10.1103/physreve.56.1171

7. Dunkel, J., Schimansky-Geier, L. and Ebeling, W. (2004) Exact solutions for evolutionary strategies on harmonic landscapes, Evolutionary computation, volume 12, number 1, 1-17. https://doi.org/10.1162/evco.2004.12.1.1

8. Pearce, P. and Woodhouse, F. G. and Forrow, A., Kelly, A. and Kusumaatmaja, H. and Dunkel, J. (2018) Inference of complex state transition networks via reconstructed high-dimensional energy landscapes, unpublished preprint.

9. Forrow, A. and Woodhouse, F. G. and Dunkel, J. (2018) Functional control of network dynamics using designed Laplacian spectra, arXiv preprint arXiv:1801.01573v1.

10. Kramers, H. A. (1940) Brownian motion in a field of force and the diffusion model of chemical reactions, Physica, volume 7, number 4, 284-304. https://doi.org/10.1016/s0031-8914(40)90098-2

\section{ABOUT THE STUDENT AUTHOR}

Sean Elliott is currently studying mathematics at Southern Methodist University. He plans to pursue a doctorate in math as well, followed by a career as a mathematician. 


\section{PRESS SUMMARY}

This research presents a mathematical model for biological evolution based on a partial differential equation known as the Fisher-Eigen (FE) process. This equation describes the evolution of a population within a space of genetically guided characteristics, or phenotypes. The FE equation also depends on the choice of a fitness function that represents the likelihood of survival at each value in the phenotype space. The FE model has been studied analytically for simple fitness functions, but in general becomes too complex to analyze or simulate when many phenotypes are included. The goal was to form a more feasible model that successfully captures the dynamics of the original but enables the inclusion of more-potentially thousands-of phenotypes. For the design, the FE process was first simulated over a 2D energy landscape using MATLAB. It was assumed that organisms could be clustered into groups that share similar genes, and then the interactions between these groups could be studied. Next, system of ordinary differential equations over the network of these groups was created and it was shown that model found the correct equilibrium solution. The result was a computationally feasible model that captures the behavior of a population over time. This prototype can be replicated by others to make modeling of this high-dimensional data feasible, allowing researchers to predict dynamic behavior based on equilibrium data. 


\title{
Assessing the Use of Cooking Demonstrations on Healthy Eating Barriers in City Bus Riders
}

\author{
Julianne Evans* \& Diana Cuy Castellanos \\ Department of Health and Sports Science, College of Education and Health Sciences, University of Dayton, OH \\ bttps://doi.org/10.33697/ajur.2019.017 \\ Student: evansj15@udayton.edu* \\ Mentor:dcuycastellanos1@udayton.edu
}

\begin{abstract}
People living in food-insecure households may experience access-related barriers to preparing and consuming fresh produce, such as high cost and limited local availability. Nutrition interventions that incorporate improved access play a decisive role in overcoming these barriers. The urban bus hub fresh produce market was developed to address food insecurity in Dayton, Ohio. Over four months, dietetic students from a four-year, private, mid-western university provided cooking demonstrations and recipe distribution nutrition interventions at the market. We used a quasi-experimental study design to determine the effectiveness of the cooking demonstration and recipe distribution intervention on access-related barriers at the urban bus hub fresh produce market. A ten-item quantitative questionnaire, on a Likert-type scale from 1-10, with 10 as a more favorable response, and four open-ended questions were administered once to each consented participant $(\mathrm{N}=33)$ to examine the recipe effectives and explore the access-related barriers. T-tests were used to examine barriers from the questionnaire and determine differences between participants who made the recipe and participants who did not make the recipe. Results of the t-test indicate no significant difference between those who made the recipe and those who did not make the recipe ( $\mathrm{p}>0.05)$. Mean scores for affordability, acceptability, accommodation, availability, and accessibility on the then ten-item questionnaire were 7.83, 8.44, 9.19, 9.38, and 8.44, respectively. Thematic analysis results were used to examine the barriers from the open-ended questions further and revealed that affordability and time barriers were present in the priority population. Lack of money, job loss, and unemployment were identified as contributing to affordability barriers and transportation and time to shop, prepare, or cook produce, and to find mealtimes were identified as contributing to time barriers. Nutrition professionals should continue developing appropriate interventions for affordability and time barriers in convenient locations for participants to encourage fruit and vegetable consumption and to establish evidence-based practices.
\end{abstract}

\section{KEYWORDS}

Cooking demonstrations; food insecurity; produce consumption barriers; nutrition intervention; food stand; recipe distribution; bus hub

\section{INTRODUCTION}

The definition of food security, as defined by the United States Department of Agriculture (USDA), is "access by all people at all times to enough food for an active, healthy lifestyle." ${ }^{1}$ Currently, concerning $11.8 \%$ of the nation reports some level of food insecurity, a household condition of uncertain or limited access to an adequate food supply. ${ }^{2}$ The state of Ohio has a food insecurity rate of $16.0 \%, 4.2 \%$ above the national average, with the city of Dayton at $18.0 \% .{ }^{2-4}$ Low socioeconomic status and low educational attainment often lead to food insecurity and may result in poor dietary behavior. ${ }^{5-8}$ Furthermore, food insecurity is a national concern due to its association with poor mental and physical health. ${ }^{5-8}$

Food insecurity is negatively associated with recommended fruit and vegetable intake, ${ }^{5-7}$ but positively related to unhealthy fat, refined sugar, and processed food intake, ${ }^{6-8}$ which may increase the risk for obesity. ${ }^{7}$ Addressing barriers related to consuming fruit and vegetables within food insecure populations could promote overall health. For instance, research indicates that fruit and vegetable consumption is associated with the ability to purchase and prepare fresh fruits and vegetables. ${ }^{5-8}$

Researchers have explored the barriers impacting fresh produce purchase, preparation, and consumption in low-income populations. The barriers relate to behaviors, attitudes, environments, education, and socioeconomic status. ${ }^{6-11}$ Environmental barriers include transportation, food cost, store locations, and hours of operation. ${ }^{9}$ Behavioral and attitudinal barriers include cooking skills, perception of time, discipline, lethargy, social values, norms, and consumer needs.7, 9-11 A few studies have connected these barriers into five access-related dimensions: availability, accessibility, affordability, accommodation, and acceptability. ${ }^{9-10}$ These five barriers were first identified by Penchansky and Thomas, as they identified a taxonomic definition for 
access as it related to healthcare systems and healthcare services. ${ }^{12}$ Caspi, Sorensen, Subramanian, and Kawachi were the first researchers to connect the five dimensions of access to the food environment and diet. ${ }^{10}$ Availability referred to locality and the supply of food, accessibility to transportation and travel time to get to the food supply, affordability to food cost, accommodation to consumer acceptance and adequacy to meet their needs, and acceptability to attitudes, including values and norms, towards the food supply. ${ }^{9-10}$ Nutrition interventions have been created, either directly or indirectly, to target the barriers associated with these five dimensions of access. $5,8,11,13-15$

Several studies assessed the impact of cooking classes and incentive programs at farmer's markets to help address barriers of affordability and acceptability. ${ }^{13-15}$ In one study, the farmer's market personnel provided a monetary incentive to help reduce produce cost and provided cooking classes to increase knowledge of fresh produce, self-efficacy, and healthy dietary behaviors. ${ }^{13}$ The focus groups and survey responses indicated the incentive program and cooking classes at the farmer's market increased fruit and vegetable consumption and positively changed their attitudes towards fruit and vegetables. ${ }^{13}$ Another study evaluated produce availability at markets and consumers knowledge of what was available through nutrition education. ${ }^{14}$ The nutrition education connected consumers, farmers, and food assistance program to evaluate the use of the fresh produce at the market. ${ }^{14}$ Availability, affordability, and accessibility barriers were also studied in senior citizen populations where they were provided with vouchers as farmer's sold their produce at established and new locations. ${ }^{14}$ Incentives and cooking classes or demonstrations are encouraging ways to increase fruit and vegetable consumption for low-income individuals by addressing the identified barriers, both environmental and behavioral. ${ }^{11,13-15}$ Furthermore, increased fruit and vegetable consumption, as a result of nutrition education paired with monetary incentives, is associated with reduced risk for food insecurity. 5,8

Another aspect of food access that is critical to understand is the concept of food deserts. A food desert is an area where there is inadequate access to healthy foods, such as fruits, vegetables, and whole grains. ${ }^{16}$ Food deserts contribute to the dimensions of access because it is a way to understand the accessibility dimensions, relating to travel and proximity of food supply. Dayton, Ohio is located within Montgomery County, where $66 \%$ of the food deserts in the county are located in the downtown area. ${ }^{17}$ Homefull, a local non-profit that addresses homelessness in the Dayton area, created a bus hub market to help address this accessibility barrier.

The market is a fresh fruit and vegetable stand located adjacent to the public transportation hub in downtown Dayton, Ohio. Homefull clients, who are part of a work entry program, oversee the market and the Homefull microfarm. ${ }^{18}$ Customers may pay for produce using Electronic Benefit Transfer (EBT), credit, or cash. In January of 2017, dietetics students from the University of Dayton began to implement weekly cooking demonstrations at the bus hub market to increase knowledge regarding fresh produce preparation, using culturally-appropriate recipes. Individuals at the bus hub could stop and watch the demonstration, sample food, speak with the students, and take a recipe card. The recipe card listed the ingredients, directions, nutrition facts, and describes health facts related to produce in the recipe. The primary researcher tailored the recipes for the priority population to be healthy, culturally appropriate, affordable, simple, and quick. The objective of this study was to assess the impact of cooking demonstrations on perceived barriers to fruit and vegetable preparation and consumption at the public transit hub produce market in downtown Dayton using quantitative and qualitative methods.

\section{METHODS AND PROCEDURES}

\section{Study Design}

This was a quasi-experimental study approved by the University of Dayton's Institutional Review Board.

\section{Participants}

The primary researcher recruited participants, using convenience sampling methods, during the cooking demonstrations at the bus hub market. Individuals who approached the cooking demonstration table took a recipe and were $\geq 18$ years old met the inclusion criteria.

\section{Setting}

The market has five to six food stands in a U-shape inside the bus hub. Centrally located is a check-out stand and on either side is one produce stand. There are 1-2 produce stands adjacent to these, making a U-shape. For the demonstrations that occurred once a week, the market manager would move the stands on the left side to create space for the portable sink and table. Additionally, one of the stands with a steel demonstration surface was used. Each surface was sanitized before use. Figure 1 indicates the set-up of the cooking demonstrations in the market. 


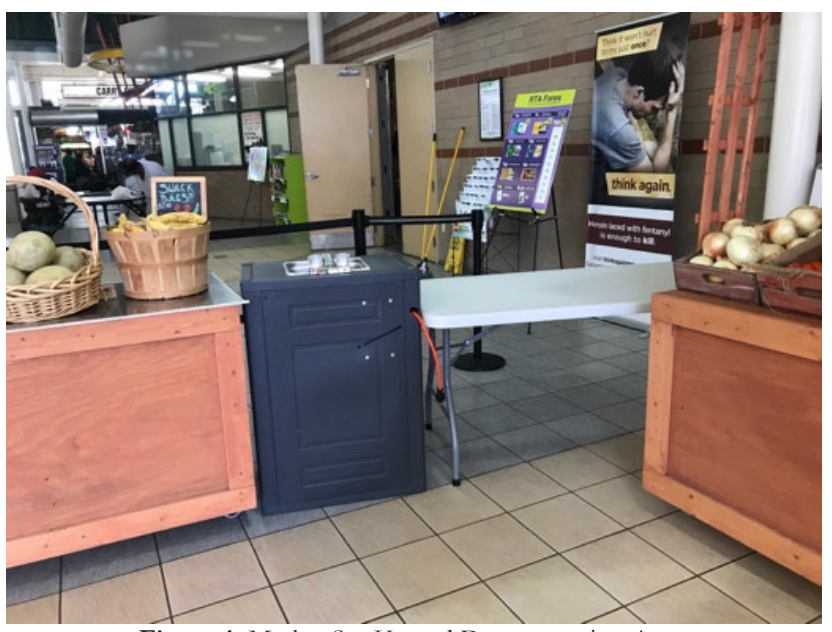

Figure 1. Market Set-Up and Demonstration Area.

\section{Intervention Procedures}

Cooking demonstrations occurred from August 2017 - February 2018, once a week in bus hub market. There was a total of 19 demonstrations and 19 recipes used. Demonstrations ranged from 1.5-2 hours, including set up and clean up time, depending on the recipe selected. The primary researcher selected a weekly recipe and performed the cooking demonstration with a trained dietetic student demonstrator, where recipe cards and samples were distributed. During the demonstrations, the dietetic students and primary researcher's engaged in casual conversation with individuals walking by or stopping to try the samples. The students and researchers would ask how people were doing and if they wanted to try the recipe. They might also answer any questions individuals had about the demonstration or recipe. Figure 2 is a sample of the distributed recipe cards to individuals walking in the bus hub. Figure 3 shows the various demonstrations and some of the recipes used in the intervention.

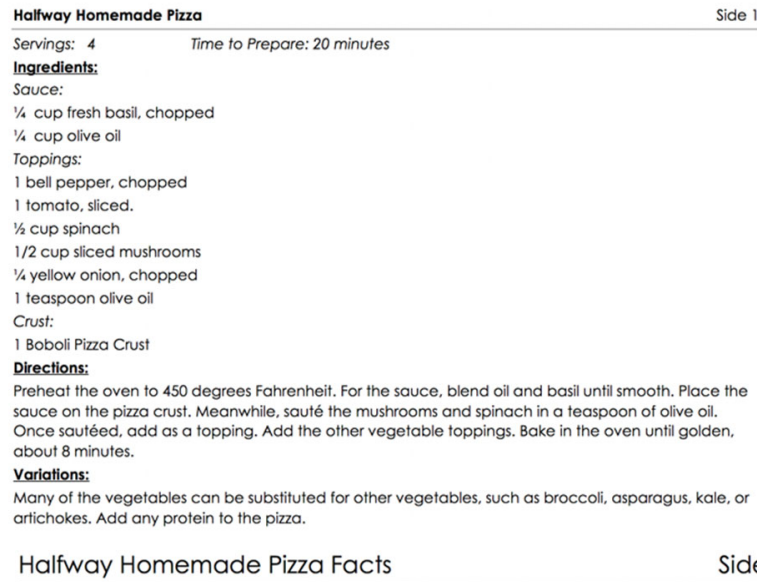

Figure 2. Recipe Card for Halfway Homemade Pizza. Side 1 has the recipe. Side 2 has the nutrition and food facts. 


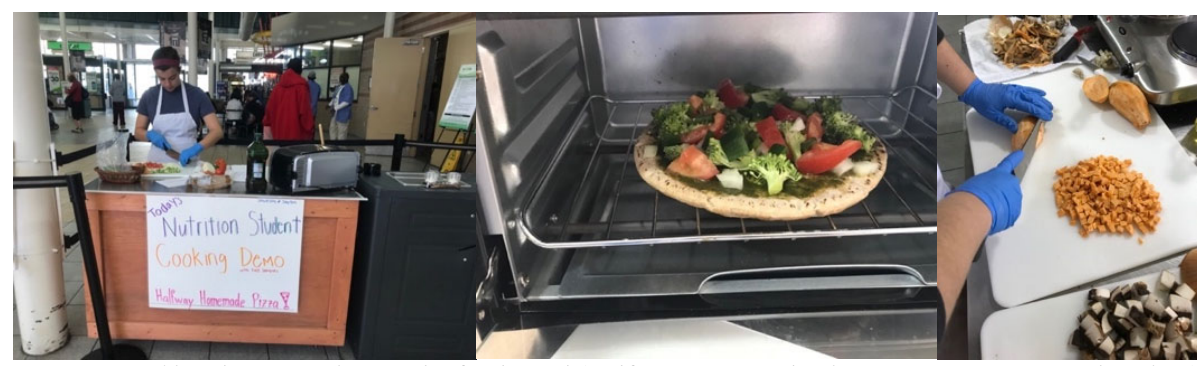

Figure 3. Cooking demonstrations at the food stand (Halfway Homemade Pizza \& Sweet Potato Hash recipes).

\section{Survey Procedures}

Survey collection occurred between August 2017 - February 2018. Through informal conversation with individuals in the bus hub about the demonstration, the primary investigator explained the research and asked if the individual would be willing to participate. If willing, the individuals signed a consent form and completed the best way to be contacted. Seven to ten days after the participant filled out the forms, the primary researcher telephoned the participant. When the participant answered the call, the primary researcher asked them questions from the questionnaire, including open-ended follow up questions.

\section{Data Instrumentation}

A questionnaire assessing the five identified barriers was adapted from a previous study examining fruit and vegetable purchase, preparation, and consumption barriers. ${ }^{9-10}$ The first item asked if participants made the recipe, which separated participants into the two groups of those who made the recipe and those who did not make the recipe. The remaining items were placed on a 1-10 Likert type scale, and participants indicated their degree of agreement with each statement, with 10 as most agreeable and 1 as least agreeable. Items 2-10 assessed the identified barriers and utilized adapted key terms listed in Table 1 to ascertain the degree of agreement.

\begin{tabular}{|l|l|l|}
\hline \multicolumn{1}{|c|}{ Item } & Barrier & Key Terms \\
\hline 2 & Affordability & Affordability of recipe \\
\hline 3 & Acceptability & Convenience of recipe \\
\hline 4 & Accommodation & Simplicity of recipe \\
\hline 5 & Affordability & Affordability of F/V \\
\hline 6 & Acceptability & Convenience of cooking F/V \\
\hline 7 & Accommodation & Simplicity of cooking F/V \\
\hline 8 & Availability & Location of market \\
\hline 9 & Accessibility & Hours market is open \\
\hline 10 & Acceptability & Knowledge of F/V preparation \\
\hline
\end{tabular}

Open-ended questions were created to gain a better understanding of the reasons participants did or did not prepare the recipe. For those who prepared the recipes, questions included describing the experience with making the recipe, such as time, recipe modification, where produce was purchased, what went well, and what did not go well. For those who did not prepare the recipes, the primary question asked was what prevented participants from making the recipe, and informal follow up questions about the barrier were asked.

\section{Statistical Analysis}

T-test, frequencies, and correlations were analyzed using IBM SPSS Ed. 23. Frequencies were calculated for gender and those who made the recipe vs. those who did not. Means and standard deviations for each of the five barriers and age were determined. Ttests were utilized to examine the mean difference between those who made the recipe versus those who did not make the recipe for each of the five barriers. Correlations were utilized to understand the relationship between recipe perception and fresh produce perception. Statistical significance was determined based on a $0.05 \alpha$ level. Thematic analysis was completed for the openended follow up questions. The access-related barrier definitions were used to identify themes in the participant responses. Keywords and phrases in the participant's responses were categorized based on which access-related barrier they spoke to provide a sense of their lived experience related to each of the barriers. 


\section{RESULTS}

Thirty-three participants completed the questionnaire, and the open-ended follow up questions. Of these participants, 21 were female, and 12 were male. The participant age range was between 20 and 66 years, with a mean age of 42.45 (SD = 14.87). Of the 33 participants, 17 made the recipe. Age and gender were collected; however, it was collected independently from the ten-item questionnaire and the four open-ended questions, so these variables could not be included in our analyses.

\section{T-Test Results}

Table 2 provides the average score for each of the five identified barriers, the t statistic, p-value, and eta-squared. Across the five categories for produce purchase, consumption, and preparation barriers, there was not a significant difference between those who made the recipes and those who did not make the recipes $(p>0.05)$. The mean score for the five barriers were all above 7.00 . Further, there was a minimal effect for each of the identified barriers.

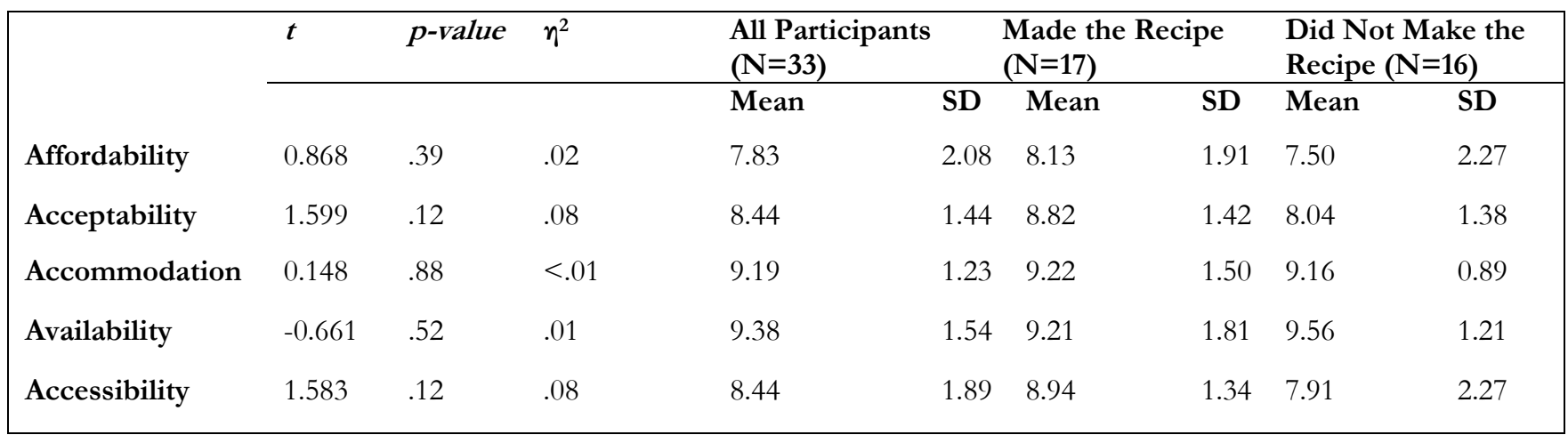

Table 2. Questionnaire Results Across Recipe Groups: mean, standard deviations, $t$-test, $p$-value, and eta-squared.

\section{Thematic Analysis Results}

The thematic analysis revealed detailed descriptions of the five identified barriers. Table 3 identifies key elements from the thematic analysis. One of the apparent barriers was affordability. Participants who made the recipe and participants who did not make the recipe indicated that it could be challenging to find the money to buy produce. Multiple factors for lack of money included low income, job loss, and unemployment. Additionally, grocery stores with competitive produce prices, such as Kroger, Aldi, and Save-A-Lot, contributed to variable produce affordability perception at the bus hub market because participants stated comparing produce prices at the Market to these grocers to find the "cheapest" produce possible.

\begin{tabular}{|c|c|c|}
\hline & Made the Recipe $(\mathrm{N}=17)$ & Did Not Make the Recipe $(\mathrm{N}=16)$ \\
\hline Affordability & $\begin{array}{l}\text { - } \quad \text { Produce is more expensive in winter } \\
\text { - Comparing produce prices to find } \\
\text { the best deal }\end{array}$ & $\begin{array}{l}\text { - Lack of money to purchase produce (no } \\
\text { income or lack of money) } \\
\text { - Comparing produce prices to find the } \\
\text { best deal }\end{array}$ \\
\hline Acceptability & - Recipes were easy to follow & $\begin{array}{l}\text { - Forgetting the recipe } \\
\text { - } \quad \text { Could not get the ingredients at the store }\end{array}$ \\
\hline Accommodation & $\begin{array}{l}\text { - } \\
\text { - } \quad \text { Easy to substitute recipes } \\
\text { ingredients with other produce } \\
\text { preferences }\end{array}$ & $\begin{array}{l}\text { - Lack of time due to work or medical } \\
\text { issues } \\
\text { Cannot cut/cook the produce in the } \\
\text { same way as performed during the } \\
\text { demonstration }\end{array}$ \\
\hline Availability & - Market is a good location & $\begin{array}{l}\text { - Market is not always close, but it is a } \\
\text { good location }\end{array}$ \\
\hline Accessibility & $\begin{array}{l}\text { - More open hours of operation } \\
\text { would be helpful }\end{array}$ & $\begin{array}{l}\text { - Lack of time to get to the hub due to a } \\
\text { job, traveling, or transportation }\end{array}$ \\
\hline
\end{tabular}

Table 3. Open-Ended Questions: Thematic Analysis Key Ideas 
Even more evident than affordability, the open-ended questions revealed that time was the most prominent barrier for those who did not make the recipe. Time prevented people from making the recipe. Time, as understood by participants, included time to find transportation to the store, shop for ingredients and produce, prepare and cook the recipe, and sit down and eat the prepared food. The barriers mentioned that required time and prevented people from making the recipe encompassed medical issues, job, travel, and transportation.

For those who made the recipe, key themes encompassed ideas on recipe simplicity and convenience, and self-efficacy of the participants. Although these did not appear to be barriers, participants mentioned that these factors helped them make the recipes on their own. Participants in this group also demonstrated a motivation to make the recipe by using phrases including a healthy lifestyle, eating more fruits and vegetables, healthy cooking, and concern for overall health.

\section{DISCUSSION}

Evaluating Barriers and the Nutrition Intervention

The purpose of the present study was to assess the impact of fresh produce cooking demonstrations and recipe distribution at an urban bus hub fresh produce market on the five identified barriers to recipe preparation and fruit and vegetable intake. Although there were no significant findings, understanding the impact of the null findings is essential for nutrition professionals. The null findings suggest the need for more evidence-based nutrition interventions for the priority population. The null findings differ from previous research, which suggests that similar target populations encounter the five identified barriers., 13, 15, 17, 19 The barriers found in previous research for low-income and food insecure populations include psychosocial, environmental, and educational barriers, encompassing the five barriers explored in this research.-9, 13, 15, 17, 19 Research indicates that addressing these barriers is vital for nutrition professionals. Studies have explored how nutrition professionals have the potential to address these barriers through gardening classes, cooking demonstrations paired with monetary incentives, and incentives paired with nutrition education. ${ }^{11,13-15}$ However, the findings from this research support the continual evaluation and re-assessment of nutrition interventions in less studied locations, such as programs in transportation hub markets, to begin developing more evidence for practice in these settings. Since food access related to transportation has been found to impact fruit and vegetable purchase and consumption, the need for interventions related to transportation might be valuable to nutrition professionals. ${ }^{1,9-11,17}$ Nutrition professionals can impact nutrition messages in these settings. $19,11,17$ This study provides a starting point for further research on nutrition interventions in unique settings.

Exploring the implications further, one common trend is the affordability barrier. Although not significant between the two groups studied, affordability had the lowest mean score across all five barriers. This is consistent with comments collected in the open-ended questions that reveal fresh produce purchase, preparation, and consumption is still largely dependent on the affordability of fresh produce. Since the cost was a central component of the thematic analysis and the lowest mean score, this demonstrates that affordability is still an important barrier to consider when understanding fresh produce perceptions and developing nutrition intervention, which is consistent with previous research.11, 14-15, 19 These studies indicate a positive relationship between monetary incentives, such as SNAP, Health Bucks, or another stipend, and perceptions of produce affordability and increased purchases and consumption of fruit and vegetables. ${ }^{11,14-15,19}$ Although the primary researcher could not directly address produce affordability by changing produce prices at the market or through incentives, the recipes were tailored to meet these needs. Ingredients were selected for their affordability, commonness in homes, and overall health quality. Additionally, the bus hub market coordinated by Homefull tried to address the affordability barrier by accepting EBT for fresh produce and offering reduced prices for some produce if it is bought in bulk amounts. Affordability of recipes is something that can be further explored in the development of nutrition interventions in settings where EBT and similar programs are already established.

In addition to the emphasis on affordability, the thematic analysis revealed that time was an important barrier for participants. Past research suggests that time is a more significant barrier when considering dietary behavior and meal preparation more so than monetary barriers, like the affordability of produce. ${ }^{20-24}$ One way time can be measured is through meal preparation time and labor cost. $^{20-24}$ Meal preparation includes items such as cooking or clean up time, while labor cost might include shopping or transportation. ${ }^{17-21}$ The labor cost and meal preparation time relates to healthy dietary behaviors because it helps to quantify the time and energy that goes into making a meal. ${ }^{20-24}$ Exploring the perception of time is an important result of this research because the null findings signify the importance of collecting more data on possible barriers this population encounters, one of the barriers being time. Furthermore, understanding time might be able to provide information on acceptability, accessibility, affordability, and accommodation barriers because they represent different aspects of labor cost. Acceptability and accommodation relate to labor cost because of their influence on dietary behaviors, including meal preparation, as related to consumer needs, values, and norms. ${ }^{9}, 20$ Accessibility and availability relate to time because of transportation and market location as part of the labor cost.9, ${ }^{20}$ The null findings of this research signify that barriers such as accessibility, availability, accommodation, and acceptability can be further explored as entities of time and labor cost that participants have. Participants 
perception of time can provide nutrition professionals with detailed information on how to overcome this barrier through effective and efficient nutrition interventions.

Another aspect to consider with null findings is that different aspects of the five barriers might already be addressed through the location of the produce market in the bus hub. The location of the market is convenient for people who use public transit, and it does not always require them to take another bus route to go to the grocery store. ${ }^{17}$ In this respect, the market is very accessible to people on the bus route. The market also accepts EBT, which could be addressing the barrier related to affordability. ${ }^{17}$ Furthermore, even the exposure to fresh produce could help reduce barriers related to acceptability, accommodation, and availability because just passing by a fresh produce market amidst other fast food options can alter social values and norms and the needs of individuals. Considering the barriers the market already addresses through their location, the null findings may indicate the market has been successful in addressing some the food insecurity issues in Dayton. ${ }^{16,17}$ The null findings may indicate that the five barriers have been addressed by the nutrition intervention, but more evidence would need to be collected to understand this possible relationship.

The cooking demonstration and recipe distribution nutrition intervention is an important aspect of continuing to address barriers to fresh produce purchase, preparation, and consumption, but the focus of the intervention should change based on the evidence from this study, indicating time as the most significant barrier the target population encounters. The null findings provide nutrition professionals with evidence that the intervention needs to change as the barriers people encounter with fresh produce purchase, preparation, and consumption change. Other researchers support the importance of continuing to disseminate nutrition information and provide education on cooking skills and produce preparation. ${ }^{7,9,11,13-15}$ Furthermore, this evidence is rooted in support from the dietetics practice that emphasizes the continual evaluation of programs as necessary for their development towards impact.

\section{Limitations}

The location of the market is in a Dayton food desert, and the market accepts EBT.14, 15 Although the study focused on food insecure individuals, we did not confirm that participants lived in a food-insecure household. Gender and age were collected, but they were independent of the questionnaire and thus could not be analyzed with the data because of the coding system used to keep participant information private. Furthermore, a sample size of 33 is another significant limitation because such a small sample is less representative of the population. These were all noteworthy limitations to participant demographics.

The Likert type scale used for the questions was broad (1-10), and participants might have benefited from small scale, which could have provided researchers with a more concise assessment of the five barriers. A pre-test and post-test could have also been used to assess the five barriers before and after the cooking demonstrations and recipe distribution. Lack of pre-testing and posttesting prevented the primary investigator from determining change and overall impact of the nutrition intervention because all participants watched the demonstrations and took the recipes. It is unclear if all participants made the recipes within a week after the demo or waited until a later date to make the recipes. In this way, the methodology limited the ability to assess the impact and outcome.

Similarly, a lack of consistency in the recipes used for the demonstrations may have reduced the ability to assess the impact and outcome. Since there were 19 different recipes for each of the 19 demonstrations, this might have created considerable variability in the ability of a participant to go home and make the recipe. Some recipes might have been easier to make at home for various reasons, contributing to varied accommodation and acceptability barriers based on the recipe. Recipes still might have also been more or less affordable, depending on what was being made. This variability in the recipes limited the ability to assess the effectiveness of the intervention. Additionally, the three dietetics students who assisted and led the cooking demonstrations with the primary researcher were all Caucasian females who built trust through the longevity of the positive interactions during the demonstrations. This trust could have contributed to some variability in responses, again, limiting the ability to assess impact.

More information on how time impacts implementing nutrition interventions and produce purchase, preparation, and consumption is essential, although it is a barrier not initially explored. ${ }^{16-20}$ A thorough understanding of participants perception on time could provide details on what drives people and prevents people from engaging with the demonstration and creating the recipes on their own.

\section{CONCLUSIONS}

It is important to continue developing nutrition interventions that can better meet the consumers' needs to increase fruit and vegetable preparation and consumption. As interventions are used at food stands and farmer's markets, it is important to continue to assess their impact on participants, evaluating how the interventions meet the consumers' needs, social values, norms, and how it increases fruit and vegetable preparation and consumption. The previous study of nutrition interventions in public transit hubs 
fresh produce markets is minimal and should continue developing. Information on the correlation between produce perception, transportation, and nutrition interventions can be further explored to help understand food insecurity and healthy lifestyles. This research serves as a pilot program for nutrition professionals to continue developing evidence-based practices on nutrition interventions at the public transit food stand, which are becoming frequent locations for nutrition education. Further ideas for this study include exploring labor cost and meal preparation time by interviewing people at the market on their perception of time-related barriers.

\section{ACKNOWLEDGMENTS}

The Stander Symposium Research Grant funded this study. We thank Homefull personnel, Dayton RTA personnel, dietetic students who administered the cooking demonstrations, and our participants for assisting in this study.

\section{REFERENCES}

1. Colemen-Jense, A, Gregory, A., \& Rabbitt, M. (2017) Overview: Food Security in the U.S., bttps:// mmw.ers.usda.gov/topics/foodnutrition-assistance/food-security-in-the-us/ (accessed Dec 2017)

2. Colemen-Jense, A, Gregory, A., \& Rabbitt, M. (2017) Key Statistics and Graphics, https://wmw.ers.usda.gov/topics/food-nutritionassistance/food-security-in-the-us/key-statistics-graphics/ (accessed Dec 2017)

3. Feeding America. (2015) Food Insecurity in Ohio, http:// map.feedingamerica.org/county/2015/overall/obio (accessed Dec 2017).

4. Feeding America. (2015) Food Insecurity in Montgomery County, http:/ / map.feedingamerica.org/county/2015/overall/obio/county/ montgomery (accessed Dec 2017).

5. Savoie-Roskos, M., Druward, C., Jeweks, M., \& LeBlanc, H. (2016) Reducing food insecurity and improving fruit and vegetable intake among farmers' market incentive program participants, J Nutr Edu Behav 48.1, 70-76. bttps:// doi.org/10.1016/j.jneb.2015.10.003

6. Hampl, J., \& Hall, R. (2002) Dietetic approaches to US hunger and food insecurity, J Am Diet Assoc, 102.7, 19-923. https:/ / doi.org/ 10.1016/s0002-8223(02)90209-9

7. Rogers, B., Kegler, M.C., Berg, C. J., Haardorfer, R., \& Frederick G.T. (2016) Understanding the food insecurity and obesity relationship by examining potential mediators: an exploratory analysis, J Hunger Environ Nutr, 11.2, 195-209. https:// doi.org/10.1080/19320248.2015.1128863

8. Jablonski, B. B. R., McFadden, D. T., \& Colpaart, A. (2016) Analyzing the role of community and individual factors in food insecurity: identifying the diverse barriers across clustered community members. J Community Health, 41, $910-923$. bttps://doi.org/10.1007/s10900-016-0171-0

9. Wetherill, M. S., \& Gray, K. A. (2015) Farmers' markets and the local food environment: identifying perceived accessibility barriers for SNAP consumers receiving temporary assistance for needy families (TANF) in an urban Oklahoma community, $J$ Nutr Educ Behav 47.2, 127-33.e1. https:/ / doi.org/10.1016/j.jneb.2014.12.008

10. Capsi, C.E., Sorensen, G. Subramanian, S.V., \& Kawachi, I. (2012) The local food environment and diet: a systematic review, Health Place, 18.5, 1172-1187. https:// doi.org/10.1016/j.healthplace.2012.05.006

11. Eikenberry, N., \& Smith, C. (2004) Healthful eating: perceptions, motivations, barriers, and promoters in low-income Minnesota communities, J Am Diet Assoc, 104.7, 1158-1161. https:// doi.org/10.1016/j.jada.2004.04.023

12. Penchansky, R., \& Thomas, J.W. (1981) The concept of access; definition and relationship to consumer satisfaction. Med Care, 19.2, 127-140. https:// doi.org/10.1097/00005650-198102000-00001

13. Dannefer, R., Abrami, A., Rapoport, R., Sriphanlop, P., Sacks, R., \& Johns, M. (2015) A mixed-methods evaluation of a SNAP-ed farmers' market-based nutrition education program, J Nutr Educ Behav, 47.6, 516-525.e1. bttps://doi.org/10.1016/j.jneb.2015.08.021

14. Caine-Bish, N., Vogliano, C., Gosky, A., \& Powell, E. (2013) Nutrition education at farmers market to increase produce consumption of consumers and clients of food assistance programs, J Nutr Educ Behav 45.4S, 22. bttps:// doi.org/10.1016/j.jneb.2013.04.060

15. Kunkel, M. E., Luccia, B., \& Moore, A. C. (2003) Evaluation of the South Carolina seniors farmers' market nutrition education program." J Am Diet Assoc, 103.7, 880-883. https:// doi.org/10.1016/s0002-8223(03)00379-1

16. CDC (Centers for Disease Control and Prevention). (2017). Food desert. bttps:// www.cdc.gov/ healthcommunication/toolstemplates/entertainmented/ tips/FoodDesert.html (accessed Sep 2018).

17. Genth, C. (2017). West Dayton food access collective impact project in 2017 Montgomery County Food Summit: Harvesting Community Insights, Dayton, $\mathrm{OH}$, United States.

18. Homefull. (N.d.). Farm markets, http:// www.homefull.org/homefull-solutions/urban-agriculture-2/markets/(Accessed April 2019)

19. Payne, G. H., Wethington, H., Olsho, L., Jemigan, J., Farris, R., \& Walker, D.K. (2015) Implementing a farmers' market incentive program: Perspectives on the New York city health bucks program, Prev Chronic Dis, 10, 1-9.

https://doi.org/10.5888/pcd10.120285 
20. ERS (Economic Research Service). (2012) U.S household food security survey module: Three-stage design, with screeners, http:// monn.ers.usda.gov/datafiles/Food_Security_in_the_United_States/Food_Security_Survey_Modules/bh2012.pdf. (accessed Aug 2018)

21. Rose, D. (2007) Food stamps, the thrifty food plan, and meal preparation: the importance of the time dimension for US nutrition policy, J Nutr Educ Behav, 39.4, 226-232. https:// doi.org/10.1016/j.jneb.2007.04.180

22. Davis, G. \& You, W. (2010) The thrifty food plan is not thrifty when labor cost is considered, J Nutr, 140, 854-857. bttps://doi.org/10.3945/jn.109.119594

23. Davis, G. \& You, W. (2011) Not enough money or not enough time to satisfy the thrifty food plan? A cost difference approach for estimating a money-time threshold, Food Policy, 36.2, 101-107. https:// doi.org/10.1016/j.foodpol.2010.09.001

24. Mancino, L. \& C. Newman. (2007) Who has time to cook? How family resources influence food preparation, https://wnw.ers.usda.gov/webdocs/publications/45797/11722_err40_1_.pdf? $v=0$ (accessed Aug 2018)

\section{ABOUT STUDENT AUTHOR}

Julianne Evans is from Kettering, OH and graduated from The University of Dayton in May 2019, majoring in dietetics and minoring in sustainability, energy, and environment. Beginning in fall 2019, she is pursuing an M.S in Public Health Nutrition and a Dietetic Internship at The University of Tennessee, Knoxville.

\section{PRESS SUMMARY}

Lack of healthy food sources prevents food-insecure people from eating and creating meals with fresh produce. Barriers that play a key role in eating and cooking healthy are socioeconomic status, psychosocial behaviors, environmental factors, and educational attainment. Nutrition programs have positively impacted these barriers. Since previous research on nutrition programs has not been completed in public transportation hubs, the goal of this research was to determine the effectiveness of a cooking demonstration and recipe distribution program at a fresh produce market in an urban bus hub. People at the bus hub and the market could watch the demonstrations and take recipes. Participants who agreed to participate in the study were called and asked questions on barriers. After evaluating participants responses to these questions, researchers found that time is still a significant barrier that prevented people from creating meals with fresh produce. It is essential to keep developing and evaluating nutrition programs in convenient locations, such as public transit hubs, so that nutrition professionals can address barriers to eating and cooking healthy meals. 
Print Edition ISSN 1536-4585

Online Edition ISSN 2375-8732 